\title{
A KAM theorem without action-angle variables for elliptic lower dimensional tori
}

\author{
Alejandro Luque Jordi Villanueva \\ February 15, 2010 \\ Departament de Matemàtica Aplicada I, \\ Universitat Politècnica de Catalunya, \\ Diagonal 647, 08028 Barcelona (Spain). \\ alejandro.luque@upc.edu jordi.villanueva@upc.edu
}

\begin{abstract}
We study elliptic lower dimensional invariant tori of Hamiltonian systems via parameterizations. The method is based in solving iteratively the functional equations that stand for invariance and reducibility. In contrast with classical methods, we do not assume that the system is close to integrable nor that is written in action-angle variables. We only require an approximation of an invariant torus of fixed vector of basic frequencies and a basis along the torus that approximately reduces the normal variational equations to constant coefficients. We want to highlight that this approach presents many advantages compared with methods which are built in terms of canonical transformations, e.g., it produces simpler and more constructive proofs that lead to more efficient numerical algorithms for the computation of these objects. Such numerical algorithms are suitable to be adapted in order to perform computer assisted proofs.
\end{abstract}

Mathematics Subject Classification: 37J40

Keywords: Elliptic invariant tori; KAM theory; Parameterization methods 


\section{Contents}

1 Introduction 2

2 General background 6

2.1 Basic notations . . . . . . . . . . . . . . . . . . 6

2.2 Invariant and approximately invariant tori $\ldots \ldots \ldots \ldots$

2.3 Linear normal behavior of invariant tori . . . . . . . . . . . . . . 10

$3 \quad$ Statement of the main result $\quad 13$

4 Overview and heuristics of the method 18

4.1 Sketch of the proof . . . . . . . . . . . . . . . . . 18

4.2 Characterization of the invariant and reducible case . . . . . . . . . . 21

5 One step of the Newton method 28

6 Proof of the main result 45

6.1 Convergence of the Newton scheme . . . . . . . . . . . . . . . . 46

6.2 Lipschitz regularity . . . . . . . . . . . . . . . . . . . . . . . . . . . . . . .

6.3 Measure of the set of excluded parameters . . . . . . . . . . . . 56

References

\section{Introduction}

Persistence of quasi-periodic solutions has been for long time a subject of remarkable importance in dynamical systems. Roughly speaking, KAM theory —named after A.N. Kolmogorov [38], V.I. Arnold [1] and J.K. Moser [46] — deals with the effect of small perturbations on dynamical systems (typically Hamiltonian) which admit invariant tori carrying quasi-periodic motion. Nowadays, KAM theory is a vast area of research that involves a large collection of methods and applications to a wide set of contexts: Hamiltonian systems, reversible systems, volume-preserving systems, symplectic maps, PDEs and lattices, just to mention a few. We refer to $[2,6,12,55]$ for different surveys or tutorials that collect many aspects of the theory and cover a large amount of bibliography.

In this work we are concerned with lower dimensional (isotropic) tori of Hamiltonian systems. Thus, let us consider a real analytic Hamiltonian system with $n$ degrees of freedom having an invariant torus of dimension $r<n$, carrying quasi-periodic dynamics with vector of basic frequencies $\omega \in \mathbb{R}^{r}$. The variational equations around such a torus correspond to a $2 n$-dimensional linear quasi-periodic system with vector of frequencies $\omega$. For this linear system we have $2 r$ trivial directions (i.e., zero eigenvalues of the reduced matrix of the system 
restricted to these directions) associated to the tangent directions of the torus and the symplectic conjugate ones (these trivial directions are usually referred as the central directions of the torus). If the remaining $2(n-r)$ directions (normal directions of the torus) are hyperbolic, we say that the torus is hyperbolic or whiskered. Hyperbolic tori are very robust under perturbations $[15,20,23,28,41]$. For example, if we consider a perturbation of the system depending analytically on external parameters, it can be established, under suitable conditions, the existence of an analytic (with respect to these parameters) family of hyperbolic tori having the same basic frequencies. In the above setting, if the torus possesses some elliptic (oscillatory) normal directions we say that it is elliptic or partially elliptic. In this case the situation is completely different, since we have to take into account combinations between basic and normal frequencies in the small divisors that appear in the construction of these tori (the corresponding non-resonance conditions are usually referred as Melnikov conditions [43, 44]). As a consequence, families of elliptic or partially elliptic invariant tori with fixed basic frequencies $\omega$ cannot be continuous in general, but they turn out to be Cantorian with respect to parameters. First rigorous proofs of existence of elliptic tori were given in [49] for $r=n-1$ and in [17, 40] for $r<n$. We refer also to $[5,6,24,31,35,36,52,54,62,64,66]$ as interesting contributions covering different points of view.

The main source of difficulty in presence of elliptic normal directions is the so-called lack of parameters problem $[6,49,63]$. Basically, since we have only as many internal parameters ("actions") as the number of basic frequencies of the torus, we cannot control simultaneously the normal ones, so we cannot prevent them from "falling into resonance". This is equivalent to say that, for a given Hamiltonian system, we cannot construct a torus with a fixed set of basic and normal frequencies because there are not enough parameters. The previous fact leads to the exclusion of a small set of these internal parameters in order to avoid resonances involving normal frequencies. To control the measure of the set of excluded parameters, it is necessary to assume that the normal frequencies "move" as a function of the internal parameters. Another possibility to overcome this problem is to apply the so-called Broer-Huitema-Takens theory (see [7]). This consists in adding as many (external) parameters as needed to control simultaneously the values of both basic and normal frequencies (this process is referred as unfolding). With this setting, we can prove that —under small perturbations - there exist invariant tori for a nearly full-measure Cantor set of parameters. The $\mathcal{C}^{\infty}$-Whitney smoothness of this construction is also established. Finally, in order to ensure the existence of invariant tori for the original system (free of parameters), one can apply the so-called Herman's method. Indeed, external parameters can be eliminated - under very weak non-degeneracy conditions - by means of an appropriate technical result concerning Diophantine approximation on submanifolds (see [6, 59, 61, 62, 63, 64]).

Another issue linked to persistence of lower dimensional invariant tori refers to reducibility of the normal variational equations (at least in the elliptic directions) which is usually asked in order to simplify the study of the linearized equations involved. In order to achieve this reducibility, it is typical to consider second order Melnikov conditions [43, 44] to control the small divisors of the cohomological equations appearing in the construction of the reduced ma- 
trix. Other approaches for studying persistence of invariant tori in the elliptic context, without second order Melnikov conditions, are discussed in Remark 3.10.

Classical methods for studying persistence of lower dimensional tori are based on canonical transformations performed on the Hamiltonian function. These methods typically deal with a perturbative setting in such a way that the problem is written as a perturbation of an "integrable" Hamiltonian (in the sense that it has a continuous family of reducible invariant tori), and take advantage of the existence of action-angle-like coordinates for the unperturbed Hamiltonian system. These coordinates play an important role in solving the cohomological equations involved

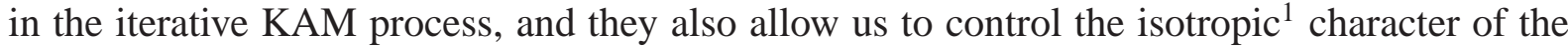
tori thus simplifying a lot of details. However, classical approaches present some shortcomings, mainly due to the fact that they only allow us to face perturbative problems. For example:

- In many practical applications (design of space missions [21, 22], study of models in Celestial Mechanics [11], Molecular Dynamics [53, 65] or Plasma-Beam Physics [45], just to mention a few) we have to consider non-perturbative systems. For such systems we can obtain approximate invariant tori by means of numerical computations or asymptotic expansions, but in general we cannot apply classical results to prove the existence of these objects. Furthermore, in some cases it is possible to identify an integrable approximation of a given system but the remaining part cannot be considered as an arbitrarily small perturbation.

- Even if we are studying a concrete perturbative problem, sometimes it is very complicated to establish action-angle variables for the unperturbed Hamiltonian. In some cases action-angle variables are not explicit, become singular or introduce problems of regularity (for example, when we approach to a separatrix). Although in many contexts this shortcoming has been solved by means of several techniques (see for example $[16,27,51]$ for a construction in the case of an integrable Hamiltonian or [8, 37] for a construction around a particular object), it introduces more technical difficulties in the problem.

- From the computational viewpoint, methods based on transformations are sometimes inefficient and quite expensive. This is a serious difficulty in order to implement numerical methods or computer assisted proofs based on them.

An alternative to the classical approach is the use of so-called parameterization methods, which consist in performing an iterative scheme to solve the invariance equation of the torus. Instead of performing canonical transformations, this scheme is carried out by adding a small function to the previous approximation of the torus. This function is obtained by solving (approximately) the linearized equation around the approximated torus (Newton method). Such approach is suitable for studying existence of invariant tori of Hamiltonian systems without using neither action-angle variables nor a perturbative setting. We point out that the geometry of

\footnotetext{
${ }^{1}$ If we pull-back an isotropic torus by means of a symplectomorphism, the isotropic character is preserved.
} 
the problem plays an important role in the study of these equations. Such geometric approach -also referred as KAM theory without action-angle variables - was introduced in [13] for Lagrangian tori and extended in [20] to hyperbolic lower dimensional tori, following long-time developed ideas (relevant work can be found in [11, 31, 47, 48, 58, 60, 67]). Roughly speaking, the insight of these methods is summarized in the following quote from [31]: “...near approximate solutions of certain equations satisfying certain non-degeneracy assumptions, we can find true solutions defined on a large set."

The aim of this paper is to adapt parameterization methods to study normally elliptic tori without using action-angle variables and in a non-perturbative setting. Concretely, we assume that we have a 1-parameter family of Hamiltonian systems for which we know a 1-parameter family of approximately invariant lower dimensional elliptic tori -all of them with the same vector of basic frequencies - and also approximations of the vectors of normal frequencies and the corresponding normal directions associated to these frequencies (i.e., a basis of the normal directions along each torus that approximately reduces the normal variational equations to constant coefficients). Then, we show that under suitable hypotheses of non-resonance and non-degeneracy, for a Cantorian subset of parameters - of large relative Lebesgue measurethere exists a true elliptic torus close to the approximate one, having the same vector of basic frequencies and slightly modified vector of normal frequencies. The scheme to deal with reducibility of the normal directions of these tori is the main contribution of this paper, and it consists in performing suitable (small) corrections in the normal directions at each step of the iterative procedure.

This setting has been selected in order to simplify some technical aspects of the result — both in the assumptions and in the proof - thus highlighting the geometric construction of the paper. We point out that all the basic ideas linked to parameterization methods, without using actionangle variables, for reducible lower-dimensional tori are present in our approach. In Section 3 we discuss several extensions and generalizations that can be tackled with the method presented in this paper.

Let us remark that parameterization methods, as presented above, are computationally oriented in the sense that they can be implemented numerically, thus obtaining very efficient algorithms for the computation of invariant tori. For example, if we approximate a torus by using $N$ Fourier modes, such algorithms allow us to compute the object with a cost of order $\mathcal{O}(N \log N)$ in time and $\mathcal{O}(N)$ in memory (see Remark 3.11). This is another advantage of our approach in contrast with classical methods based on transformation theory. The reader interested in such algorithms is referred to [14] for the implementation of the ideas in [13, 20] for Lagrangian and whiskered tori (see also [9] for the case of lattices and twist maps) and to [32] for the implementation of the ideas of [34] for reducible elliptic and hyperbolic tori for quasi-periodic skew-product maps (in this case, which corresponds to quasi-periodic perturbations of equilibrium points for flows, the geometric part discussed in the present paper is not required).

Finally, we observe that in presence of hyperbolic directions one can approach the problem by combining techniques in [20] (for studying hyperbolic directions) together with those 
introduced here (for studying elliptic directions). Indeed, the methodology presented in this work can be adapted to deal with invariant tori with reducible hyperbolic directions, but this assumption is quite restrictive (see [25]) in the hyperbolic context (reducibility is not required in [20]).

The paper is organized as follows. In Section 2 we provide some notations, definitions and background of the problem. In Section 3 we state the main result of this paper and we discuss several extensions and generalizations of the method presented. A motivating sketch of the construction performed in the proof of this result is given in Section 4, together with a detailed description of some geometric properties of elliptic lower dimensional invariant tori of Hamiltonian systems. Next, in Section 5 we perform one step of the iterative method to correct both an approximation of an elliptic invariant torus and a basis along this torus that approximately reduces the normal variational equations to constant coefficients. The new errors in invariance and reducibility are quadratic in terms of the previous ones. The main result is proved in Section 6.

\section{General background}

In this section we introduce some notation and, in order to help the reader, we recall the basic terminology and concepts related to the problem. Thus, after setting the notation used along the paper in Section 2.1, we provide the basic definitions regarding lower dimensional invariant tori of Hamiltonian systems (Section 2.2) and their normal behavior (Section 2.3).

\subsection{Basic notations}

Given a real or complex function $f$ of several variables, we denote $D f$ the Jacobian matrix, $\operatorname{grad} f=D f^{\top}$ the gradient vector and hess $f=D^{2} f$ the Hessian matrix, respectively.

For any complex number $z \in \mathbb{C}$ we denote $z^{*} \in \mathbb{C}$ its complex conjugate number and $\operatorname{Re}(z), \operatorname{Im}(z)$ the real and imaginary parts of $z$, respectively. We extend these notations to complex vectors and matrices.

Given a complex vector $v \in \mathbb{C}^{l}$ we denote by $\operatorname{diag}(v) \in M_{l \times l}(\mathbb{C})$ the diagonal matrix having the components of $v$ in the diagonal. Moreover, given $Z \in M_{l \times l}(\mathbb{C})$, we denote by $\operatorname{diag}(Z) \in M_{l \times l}(\mathbb{C})$ the diagonal matrix having the same diagonal entries as $Z$.

For any $k \in \mathbb{Z}^{r}$, we denote $|k|_{1}=\left|k_{1}\right|+\ldots+\left|k_{r}\right|$. Given a vector $x \in \mathbb{C}^{l}$, we set $|x|=\sup _{j=1, \ldots, l}\left|x_{j}\right|$ for the supremum norm and we extend the notation to the induced norm for complex matrices. Furthermore, given an analytic function $f$, with bounded derivatives in a complex domain $\mathcal{U} \subset \mathbb{C}^{l}$, and $m \in \mathbb{N}$ we introduce the $\mathcal{C}^{m}$-norm for $f$ as

$$
\|f\|_{\mathcal{C}^{m}, \mathcal{U}}=\sup _{\substack{k \in(\mathbb{N} \cup\{0\})^{l} \\ 0 \leq|k|_{1} \leq m}} \sup _{z \in \mathcal{U}}\left|D^{k} f(z)\right| .
$$


We denote by $\mathbb{T}^{r}=\mathbb{R}^{r} /(2 \pi \mathbb{Z})^{r}$ the real $r$-dimensional torus, with $r \geq 1$. We use the $|\cdot|$-norm introduced above to define the complex strip around $\mathbb{T}^{r}$ of width $\rho>0$ as

$$
\Delta(\rho)=\left\{\theta \in \mathbb{C}^{r} /(2 \pi \mathbb{Z})^{r}:|\operatorname{Im}(\theta)| \leq \rho\right\}
$$

Accordingly we will consider the Banach space of analytic functions $f: \Delta(\rho) \rightarrow \mathbb{C}$ equipped with the norm

$$
\|f\|_{\rho}=\sup _{\theta \in \Delta(\rho)}|f(\theta)| .
$$

Similarly, if $f$ takes values in $\mathbb{C}^{l}$, we set $\|f\|_{\rho}=\left|\left(\left\|f_{1}\right\|_{\rho}, \ldots,\left\|f_{l}\right\|_{\rho}\right)\right|$. If $f$ is a matrix valued function, we extend $\|f\|_{\rho}$ by computing the $|\cdot|$-norm of the constant matrix defined by the $\|\cdot\|_{\rho^{-}}$ norms of the entries of $f$. We observe that if the matrix product is defined then this space is a Banach algebra and we have $\left\|f_{1} f_{2}\right\|_{\rho} \leq\left\|f_{1}\right\|_{\rho}\left\|f_{2}\right\|_{\rho}$. In addition, we can use Cauchy estimates

$$
\left\|\frac{\partial f}{\partial \theta_{j}}\right\|_{\rho-\delta} \leq \frac{\|f\|_{\rho}}{\delta}, \quad j=1, \ldots, r .
$$

For any function $f$ analytic on $\mathbb{T}^{r}$ and taking values in $\mathbb{C}, \mathbb{C}^{l}$ or in a space of complex matrices, we denote its Fourier series as

$$
f(\theta)=\sum_{k \in \mathbb{Z}^{n}} \hat{f}_{k} \mathrm{e}^{\mathrm{i}\langle k, \theta\rangle}, \quad \hat{f}_{k}=\frac{1}{(2 \pi)^{r}} \int_{\mathbb{T}^{r}} f(\theta) \mathrm{e}^{-\mathrm{i}\langle k, \theta\rangle} d \theta
$$

and its average as $[f]_{\mathbb{T}^{r}}=\hat{f}_{0}$. We also set $\tilde{f}(\theta)=f(\theta)-[f]_{\mathbb{T}^{r}}$. Moreover, we have the following bounds

$$
\left|[f]_{\mathbb{T}^{r}}\right| \leq\|f\|_{\rho}, \quad\|\tilde{f}\|_{\rho} \leq 2\|f\|_{\rho}, \quad\left|\hat{f}_{k}\right| \leq\|f\|_{\rho} \mathrm{e}^{-\rho|k|_{1}} .
$$

Now, we introduce some notation regarding Lipschitz regularity. Assume that $f(\mu)$ is a function defined for $\mu \in I \subset \mathbb{R}$ - the subset $I$ may not be an interval— taking values in $\mathbb{C}, \mathbb{C}^{l}$ or $M_{l_{1} \times l_{2}}(\mathbb{C})$. We say that $f$ is Lipschitz with respect to $\mu$ on the set $I$ if

$$
\operatorname{Lip}_{I}(f)=\sup _{\substack{\mu_{1}, \mu_{2} \in I \\ \mu_{1} \neq \mu_{2}}} \frac{\left|f\left(\mu_{2}\right)-f\left(\mu_{1}\right)\right|}{\left|\mu_{2}-\mu_{1}\right|}<\infty
$$

The value $\operatorname{Lip}_{I}(f)$ is called the Lipschitz constant of $f$ on $I$. For these functions we define $\|f\|_{I}=\sup _{\mu \in I}|f(\mu)|$. Similarly, if we have a family $\mu \in I \subset \mathbb{R} \mapsto f_{\mu}$, where $f_{\mu}$ is a function on $\mathbb{T}^{r}$ taking values in $\mathbb{C}, \mathbb{C}^{l}$ or $M_{l_{1} \times l_{2}}(\mathbb{C})$, we extend the previous notations as

$$
\operatorname{Lip}_{I, \rho}(f)=\sup _{\substack{\mu_{1}, \mu_{2} \in I \\ \mu_{1} \neq \mu_{2}}} \frac{\left\|f_{\mu_{2}}-f_{\mu_{1}}\right\|_{\rho}}{\left|\mu_{2}-\mu_{1}\right|}<\infty, \quad\|f\|_{I, \rho}=\sup _{\mu \in I}\left\|f_{\mu}\right\|_{\rho}
$$


Analogously, given a family $\mu \in I \subset \mathbb{R} \mapsto f_{\mu}$, where $f_{\mu}$ is an analytic function with bounded derivatives in a complex domain $\mathcal{U} \subset \mathbb{C}^{l}$, we introduce for $m \in \mathbb{N}$

$$
\operatorname{Lip}_{I, \mathcal{C}^{m}, \mathcal{U}}(f)=\sup _{\substack{\mu_{1}, \mu_{2} \in I \\ \mu_{1} \neq \mu_{2}}} \frac{\left\|f_{\mu_{1}}-f_{\mu_{2}}\right\|_{\mathcal{C}^{m}, \mathcal{U}}}{\left|\mu_{1}-\mu_{2}\right|}, \quad\|f\|_{I, \mathcal{C}^{m}, \mathcal{U}}=\sup _{\mu \in I}\left\|f_{\mu}\right\|_{\mathcal{C}^{m}, \mathcal{U}} .
$$

Finally, we say that $f$ is Lipschitz from below with respect to $\mu$ on the set $I$ if

$$
\operatorname{lip}_{I}(f)=\inf _{\substack{\mu_{1}, \mu_{2} \in I \\ \mu_{1} \neq \mu_{2}}} \frac{\left|f\left(\mu_{2}\right)-f\left(\mu_{1}\right)\right|}{\left|\mu_{2}-\mu_{1}\right|}<\infty .
$$

In this work we are concerned with Hamiltonian systems in $\mathbb{R}^{2 n}$ with respect to the standard symplectic form $\Omega^{0}$, given by $\Omega^{0}(\xi, \eta)=\xi^{\top} J_{n} \eta$ where

$$
J_{n}=\left(\begin{array}{cc}
0 & \operatorname{Id}_{n} \\
-\operatorname{Id}_{n} & 0
\end{array}\right)
$$

is the canonical skew-symmetric matrix. We extend the notation above to write $J_{j}$ for any $1 \leq j \leq n$, and $\operatorname{Id}_{j}$ for any $1 \leq j \leq 2 n$. For the sake of simplicity, we denote $J=J_{n}$ and $\mathrm{Id}=\mathrm{Id}_{2 n}$.

Finally, given matrix-valued functions $A: \mathbb{T}^{r} \rightarrow M_{2 n \times l_{a}}(\mathbb{C})$ and $B: \mathbb{T}^{r} \rightarrow M_{2 n \times l_{b}}(\mathbb{C})$, we set the notations $G_{A, B}(\theta)=A(\theta)^{\top} B(\theta), \Omega_{A, B}(\theta)=A(\theta)^{\top} J B(\theta), G_{A}(\theta)=G_{A, A}(\theta)$ and $\Omega_{A}(\theta)=\Omega_{A, A}(\theta)$.

\subsection{Invariant and approximately invariant tori}

Given a Hamiltonian function $h: U \subset \mathbb{R}^{2 n} \rightarrow \mathbb{R}$, we study the existence of lower dimensional quasi-periodic invariant tori for the Hamiltonian vector field $X_{h}(x)=J \operatorname{grad} h(x)$.

Definition 2.1. For any integer $1 \leq r \leq n$, $\mathscr{T} \subset U$ is an $r$-dimensional quasi-periodic invariant torus with basic frequencies $\omega \in \mathbb{R}^{r}$ for $X_{h}$, if $\mathscr{T}$ is invariant under the flow of $X_{h}$ and there exists a parameterization given by an embedding $\tau: \mathbb{T}^{r} \rightarrow U$ such that $\mathscr{T}=\tau\left(\mathbb{T}^{r}\right)$, making the following diagram commute

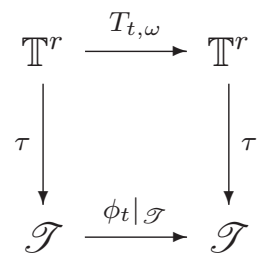

where $T_{t, \omega}(x)=x+\omega t$ is the (parallel) flow of the constant vector field

$$
L_{\omega}=\omega_{1} \frac{\partial}{\partial \theta_{1}}+\ldots+\omega_{r} \frac{\partial}{\partial \theta_{r}}
$$


and $\phi_{t}$ is the flow of the Hamiltonian vector field $X_{h}$. In addition, if

$$
\langle k, \omega\rangle \neq 0, \quad \forall k \in \mathbb{Z}^{r} \backslash\{0\},
$$

then we say that $\omega$ is non-resonant.

If $\omega \in \mathbb{R}^{r}$ is non-resonant, then the quasi-periodic function $z(t)=\tau\left(\omega t+\theta_{0}\right)$ is an integral curve of $X_{h}$ for any $\theta_{0} \in \mathbb{T}^{n}$ that fills densely $\mathscr{T}$. Equivalently, we have that the embedding $\tau$ satisfies

$$
L_{\omega} \tau(\theta)=X_{h}(\tau(\theta)) .
$$

By means of $\tau$ we can pull-back to $\mathbb{T}^{r}$ both the restrictions to $\mathscr{T}$ of the standard metric and the symplectic structure, obtaining the following matrix representations

$$
G_{D \tau}(\theta)=D \tau(\theta)^{\top} D \tau(\theta), \quad \Omega_{D \tau}(\theta)=D \tau(\theta)^{\top} J D \tau(\theta), \quad \theta \in \mathbb{T}^{r}
$$

Remark 2.2. We note that as $\tau$ is an embedding we have $\operatorname{rank}(D \tau(\theta))=r$ for every $\theta \in \mathbb{T}^{r}$, so it turns out that $\operatorname{det} G_{D \tau}(\theta) \neq 0$ for every $\theta \in \mathbb{T}^{r}$. Moreover, we see that the average $\left[\Omega_{D \tau}\right]_{\mathbb{T}^{r}}$ is zero since if we write $\tau(\theta)=(x(\theta), y(\theta))$ then we have $\Omega_{D \tau}(\theta)=D \alpha(\theta)-D \alpha(\theta)^{\top}$, where $\alpha(\theta)=D x(\theta)^{\top} y(\theta)$ and, by definition, $[D \alpha]_{\mathbb{T}^{r}}=0$.

Lemma 2.3. Let $h: U \subset \mathbb{R}^{2 n} \rightarrow \mathbb{R}$ be a Hamiltonian function and $\mathscr{T}$ an $r$-dimensional invariant torus for $X_{h}$ of non-resonant frequencies $\omega$. Then the submanifold $\mathscr{T}$ is isotropic, i.e., $\Omega_{D \tau}(\theta)=0$ for every $\theta \in \mathbb{T}^{r}$. In particular, if $r=n$ then $\mathscr{T}$ is Lagrangian.

Remark 2.4. Along the text there appear many functions depending on $\theta \in \mathbb{T}^{r}$. In order to simplify the notation sometimes we omit the dependence on $\theta$-eventually we even omit the fact that some functions are evaluated at $\tau(\theta)$ if there is no source of confusion.

Proof of Lemma 2.3. The isotropic character of $\mathscr{T}$ is obtained as it was done in [13]. First, we compute

$$
\begin{aligned}
L_{\omega}\left(\Omega_{D \tau}\right) & =L_{\omega}\left(D \tau^{\top} J D \tau\right)=\left[D\left(L_{\omega} \tau\right)\right]^{\top} J D \tau+D \tau^{\top} J D\left(L_{\omega} \tau\right) \\
& =[J \operatorname{hess} h(\tau) D \tau]^{\top} J D \tau+D \tau^{\top} J J \operatorname{hess} h(\tau) D \tau=0,
\end{aligned}
$$

where we used that $D \circ L_{\omega}=L_{\omega} \circ D$, the hypothesis $L_{\omega} \tau(\theta)=X_{h}(\tau(\theta))$ and the properties $J^{\top}=-J$ and $J^{2}=-\mathrm{Id}$. Then, since $\omega$ is non-resonant, the fact that the derivative $L_{\omega}$ vanishes implies that $\Omega_{D \tau}=\left[\Omega_{D \tau}\right]_{\mathbb{T}^{r}}$. Finally, from Remark 2.2 we conclude that $\Omega_{D \tau}=0$.

Finally, we set the idea of parameterization of an approximately invariant torus. Essentially, we measure how far to commute is diagram (1). 
Definition 2.5. Given a Hamiltonian $h: U \subset \mathbb{R}^{2 n} \rightarrow \mathbb{R}$ and an integer $1 \leq r \leq n$, we say that $\mathscr{T} \subset U$ is an r-dimensional approximately quasi-periodic invariant torus with non-resonant basic frequencies $\omega \in \mathbb{R}^{r}$ for $X_{h}$ provided that there exists an embedding $\tau: \mathbb{T}^{r} \rightarrow U$, such that $\mathscr{T}=\tau\left(\mathbb{T}^{r}\right)$, satisfying

$$
L_{\omega} \tau(\theta)=\operatorname{Jgrad} h(\tau(\theta))+e(\theta)
$$

where $e: \mathbb{T}^{r} \rightarrow \mathbb{R}^{2 n}$ is "small" in a suitable norm.

Among the conditions needed to find a true invariant torus around an approximately invariant one, we are concerned with Diophantine conditions on the vector of basic frequencies.

Definition 2.6. We say that $\omega \in \mathbb{R}^{r}$ satisfies Diophantine conditions of $(\gamma, \nu)$-type, for $\gamma>0$ and $\nu>r-1$, if

$$
|\langle k, \omega\rangle| \geq \frac{\gamma}{|k|_{1}^{\nu}}, \quad k \in \mathbb{Z}^{r} \backslash\{0\} .
$$

It is well-known that if we consider a fixed $\nu$ then, for almost every $\omega \in \mathbb{R}^{r}$, there is $\gamma>0$ for which (4) is fulfilled (see [42]).

\subsection{Linear normal behavior of invariant tori}

In order to study the behavior of the solutions in a neighborhood of an $r$-dimensional quasiperiodic invariant torus of basic frequencies $\omega$-parameterized by $\tau$ - it is usual to consider the variational equations around the torus, given by

$$
L_{\omega} \xi(\theta)=J \operatorname{hess} h(\tau(\theta)) \xi(\theta) .
$$

If $r=1$ the system (5) is $2 \pi / \omega$-periodic. Then, following Floquet's theorem, there exists a linear periodic change of variables that reduces the system to constants coefficients. If $r>$ 1 , then we consider reducibility to constant coefficients (in the sense of Lyapunov-Perron) as follows.

Definition 2.7. We say that the invariant torus $\mathscr{T}$ in Definition 2.1 is reducible if there exists a linear change of coordinates $\xi=M(\theta) \eta$, defined for $\theta \in \mathbb{T}^{r}$, such that the variational equations (5) turn out to be $L_{\omega} \eta(\theta)=B \eta(\theta)$, where $B \in M_{2 n \times 2 n}(\mathbb{C})$.

It is immediate to check that this property is equivalent to the fact that $M$ satisfies the differential equation

$$
L_{\omega} M(\theta)=J \text { hess } h(\tau(\theta)) M(\theta)-M(\theta) B \text {. }
$$

In the Lagrangian case $r=n$, under regularity assumptions, such transformation exists provided $\omega$ satisfies (4) due to the geometric constrains of the problem (see [13]). Indeed, we can take derivatives at both sides of the invariance equation (3), thus obtaining

$$
L_{\omega} D \tau(\theta)=J \operatorname{hess} h(\tau(\theta)) D \tau(\theta) .
$$


Then, we can choose a suitable $n \times n$ matrix $C(\theta)$ - given by the solution of certain cohomological equation - in such a way that the columns of $D \tau(\theta)$ and $J D \tau(\theta) G_{D \tau}^{-1}(\theta)+D \tau(\theta) C(\theta)$ give us the matrix $M(\theta)$. The reduced matrix turns out to be of the form

$$
B=\left(\begin{array}{cc}
0 & B^{C} \\
0 & 0
\end{array}\right)
$$

where $B^{C} \in M_{n \times n}(\mathbb{R})$ is symmetric. The $2 n$ zero eigenvalues correspond to the tangent directions to the torus together with their symplectic conjugate ones, meanwhile the matrix $B^{C}$ controls the variation of the frequencies of the torus - the twist condition reads $\operatorname{det} B^{C} \neq 0$ when moving the "actions" of the system.

In the lower dimensional case $1<r<n$ we cannot guarantee, in general, reducibility to constant coefficients (we refer to $[29,30,56]$ ). Nevertheless, if we consider a family of quasi-periodic linear perturbations of a linear system with constant coefficients then, under some generic hypothesis of non-resonance and non-degeneracy, we can state the reducibility of a large subfamily. On the one hand, if we restrict $M(\theta)$ to the space of close-to-the-identity matrices, then we can prove that the reducible subfamily is Cantorian and has large Lebesgue measure (we refer to $[33,34]$ ). On the other hand, considering a more general class of matrices (see ideas introduced in $[18,19,39,50]$ ) this result can be extended to a full measure subfamily (this was conjectured in [19] and proved in [26]).

If the system (5) is reducible, it turns out that the geometry of the problem allows us to choose the matrix $B$ with the following block structure

$$
B=\left(\begin{array}{cc|c}
0 & B^{C} & 0 \\
0 & 0 & 0 \\
\hline 0 & 0 & B^{N}
\end{array}\right),
$$

where $B^{C} \in M_{r \times r}(\mathbb{R})$ is symmetric (it plays the same "twist" role as in the Lagrangian case), and $B^{N} \in M_{2(n-r) \times 2(n-r)}(\mathbb{C})$ can be written as $B^{N}=J_{n-r} S$, where $S$ is also symmetric. In this context, $B^{N}$ gives the normal linear behavior of the torus. The real parts of the eigenvalues of $B^{N}$ correspond to Lyapunov exponents and their imaginary parts to normal frequencies. As discussed in the introduction, in this work we are interested in the normally elliptic case, in which all the eigenvalues of $B^{N}$ have vanishing real part, i.e.,

$$
\operatorname{spec}\left(B^{N}\right)=\left\{\mathrm{i} \lambda_{1}, \ldots, \mathrm{i} \lambda_{n-r},-\mathrm{i} \lambda_{1}, \ldots,-\mathrm{i} \lambda_{n-r}\right\},
$$

where $\lambda_{j} \in \mathbb{R} \backslash\{0\}$ are the so-called normal frequencies. Thoughtout the paper we assume that they have different modulus.

In order to simplify the resolution of the obtained cohomological equations, it is convenient to put the matrix $B^{N}$ in diagonal form. In the classical KAM approach —using symplectic transformations and action-angle variables adapted to the torus - this is possible with a complex canonical change of coordinates, that transforms the initial real Hamiltonian into a complex 
one, having some symmetries. As these symmetries are preserved by the canonical transformations performed along these classical proofs, the final Hamiltonian can be realified and thus the obtained tori are real. In this paper we perform this complexification by selecting a complex matrix function $N: \mathbb{T}^{r} \rightarrow M_{2 n \times(n-r)}(\mathbb{C})$ associated to the eigenfunctions of eigenvalues $\mathrm{i} \lambda_{1}, \ldots, \mathrm{i} \lambda_{n-r}$. It is clear that the real and imaginary parts of these vectors span the associated real normal subspace at any point of the torus. Indeed, from Equation (6), the matrix function $N$ satisfies

$$
L_{\omega} N(\theta)=J \text { hess } h(\tau(\theta)) N(\theta)-N(\theta) \Lambda,
$$

where $\Lambda=\operatorname{diag}(\mathrm{i} \lambda)=\operatorname{diag}\left(\mathrm{i} \lambda_{1}, \ldots, \mathrm{i} \lambda_{n-r}\right)$. Then, together with these vectors, we resort to the use of the complex conjugate ones, that clearly satisfy

$$
L_{\omega} N^{*}(\theta)=\operatorname{Jhess} h(\tau(\theta)) N^{*}(\theta)+N^{*}(\theta) \Lambda,
$$

to span a basis of the complexified normal space along the torus (this is guaranteed by the conditions $\operatorname{det} G_{N, N^{*}} \neq 0$ on $\mathbb{T}^{r}$ ).

As we have pointed out in the introduction of the paper, in order to face the resolution of the cohomological equations standing for invariance and reducibility of elliptic tori, we assume additional non-resonance conditions apart from (2).

Definition 2.8. We say that the normal frequencies $\lambda \in \mathbb{R}^{n-r}$ are non-resonant with respect to $\omega \in \mathbb{R}$ if

$$
\langle k, \omega\rangle+\lambda_{i} \neq 0, \quad \forall k \in \mathbb{Z}^{r}, \quad i=1, \ldots, n-r,
$$

and

$$
\langle k, \omega\rangle+\lambda_{i} \pm \lambda_{j} \neq 0, \quad \forall k \in \mathbb{Z}^{r} \backslash\{0\}, \quad i, j=1, \ldots, n-r .
$$

Conditions (7) and (8) are referred as first and second order Melnikov conditions, respectively (see $[43,44])$.

In the spirit of Definition 2.5, we introduce the idea of approximate reducibility as follows.

Definition 2.9. We say that the approximately invariant torus $\mathscr{T}$ in Definition 2.5 is approximately elliptic if there exists a map $N: \mathbb{T}^{r} \rightarrow M_{2 n \times(n-r)}(\mathbb{C})$ and normal frequencies $\lambda \in \mathbb{R}^{n-r}$, which are non-resonant with respect to $\omega$, satisfying

$$
L_{\omega} N(\theta)=J \text { hess } h(\tau(\theta)) N(\theta)-N(\theta) \Lambda+R(\theta),
$$

where $\Lambda=\operatorname{diag}(\mathrm{i} \lambda)$, $\operatorname{det} G_{N, N^{*}} \neq 0$ on $\mathbb{T}^{r}$ and $R: \mathbb{T}^{r} \rightarrow M_{2 n \times(n-r)}(\mathbb{C})$ is "small" in a suitable norm.

In order to avoid the effect of the small divisors associated to (7) and (8), we assume additional Diophantine conditions. 
Definition 2.10. Let us consider non-resonant basic and normal frequencies $(\omega, \lambda) \in \mathbb{R}^{r} \times \mathbb{R}^{n-r}$ and constants $\gamma>0$ and $\nu>r-1$. We say that $\lambda$ satisfies Diophantine conditions of $(\gamma, \nu)$-type with respect to $\omega$ if

$$
\left|\langle k, \omega\rangle+\lambda_{i}\right| \geq \frac{\gamma}{|k|_{1}^{\nu}}, \quad\left|\langle k, \omega\rangle+\lambda_{i} \pm \lambda_{j}\right| \geq \frac{\gamma}{|k|_{1}^{\nu}}
$$

$\forall k \in \mathbb{Z}^{r} \backslash\{0\}$ and $i, j=1, \ldots, n-r$.

\section{Statement of the main result}

In this section we state the main result of the paper. Concretely, if we have a 1-parameter family of Hamiltonian systems for which we know a family of parameterizations of approximately (with small error) elliptic lower dimensional invariant tori, all with the same basic frequencies and satisfying certain non-degeneracy conditions, then we use the parameter to control the normal frequencies in order to prove that there exists a large set of parameters for which we have a true elliptic invariant torus close to the approximate one. We emphasize that we do not assume that the system is given in action-angle-like coordinates nor that the Hamiltonians are close to integrable.

Theorem 3.1. Let us consider a family of Hamiltonians $\mu \in I \subset \mathbb{R} \mapsto h_{\mu}$ with $h_{\mu}: U \subset$ $\mathbb{R}^{2 n} \rightarrow \mathbb{R}$, where $I$ is a finite interval and $U$ is an open set. Let $\omega \in \mathbb{R}^{r}$ be a vector of basic frequencies satisfying Diophantine conditions (4) of $(\hat{\gamma}, \hat{\nu})$-type, with $\hat{\gamma}>0$ and $\hat{\nu}>r-1$. Assume that the following hypotheses hold:

$\mathrm{H}_{1}$ The functions $h_{\mu}$ are real analytic and can be holomorphically extended to some complex neighborhood $\mathcal{U}$ of $U$. Moreover, we assume that $\|h\|_{I, \mathcal{C}^{4}, \mathcal{U}} \leq \sigma_{0}$.

$\mathrm{H}_{2}$ There exists a family of approximate invariant and elliptic tori of $h_{\mu}$ in the sense of Definitions 2.5 and 2.9, i.e., we have families of embeddings $\mu \in I \subset \mathbb{R} \mapsto \tau_{\mu}$, matrix functions $\mu \in I \subset \mathbb{R} \mapsto N_{\mu}$ and approximated normal eigenvalues $\Lambda_{\mu}=\operatorname{diag}\left(\mathrm{i} \lambda_{\mu}\right)$, with $\lambda_{\mu} \in \mathbb{R}^{n-r}$, satisfying

$$
\begin{aligned}
L_{\omega} \tau_{\mu}(\theta) & =\operatorname{Jgrad} h_{\mu}\left(\tau_{\mu}(\theta)\right)+e_{\mu}(\theta), \\
L_{\omega} N_{\mu}(\theta) & =J \operatorname{hess} h_{\mu}\left(\tau_{\mu}(\theta)\right) N_{\mu}(\theta)-N_{\mu}(\theta) \Lambda_{\mu}+R_{\mu}(\theta),
\end{aligned}
$$

for certain error functions $e_{\mu}$ and $R_{\mu}$, where $\tau_{\mu}$ and $N_{\mu}$ are analytic and can be holomorphically extended to $\Delta(\rho)$ for certain $0<\rho<1$, satisfying $\tau_{\mu}(\Delta(\rho)) \subset \mathcal{U}$. Assume also that we have constants $\sigma_{1}, \sigma_{2}$ such that

$$
\|D \tau\|_{I, \rho},\|N\|_{I, \rho},\left\|G_{D \tau}^{-1}\right\|_{I, \rho},\left\|G_{N, N^{*}}^{-1}\right\|_{I, \rho}<\sigma_{1}, \quad \operatorname{dist}\left(\tau_{\mu}(\Delta(\rho)), \partial \mathcal{U}\right)>\sigma_{2}>0
$$

for every $\mu \in I$, where $\partial \mathcal{U}$ stands for the boundary of $\mathcal{U}$. 
$\mathrm{H}_{3}$ We have diag $\left[\Omega_{N_{\mu}, N_{\mu}^{*}}\right]_{\mathbb{T}^{r}}=\mathrm{i} \operatorname{Id}_{n-r}$ for every $\mu \in I$.

$\mathrm{H}_{4}$ The family of matrix functions

$$
A_{1, \mu}(\theta)=G_{D \tau_{\mu}}^{-1}(\theta) D \tau_{\mu}(\theta)^{\top}\left(T_{1, \mu}(\theta)+T_{2, \mu}(\theta)+T_{2, \mu}(\theta)^{\top}\right) D \tau_{\mu}(\theta) G_{D \tau_{\mu}}^{-1}(\theta),
$$

where

$$
\begin{aligned}
& T_{1, \mu}(\theta)=J^{\top} \text { hess } h_{\mu}\left(\tau_{\mu}(\theta)\right) J-\operatorname{hess} h_{\mu}\left(\tau_{\mu}(\theta)\right), \\
& T_{2, \mu}(\theta)=T_{\mu, 1}(\theta) J\left[D \tau_{\mu}(\theta) G_{D \tau_{\mu}}(\theta)^{-1} D \tau_{\mu}(\theta)^{\top}-\mathrm{Id}\right] \operatorname{Re}\left(\mathrm{i} N_{\mu}(\theta) N_{\mu}^{*}(\theta)^{\top}\right),
\end{aligned}
$$

satisfies the non-degeneracy (twist) condition $\left\|\left[A_{1}\right]_{\mathbb{T} r}^{-1}\right\|_{I}<\sigma_{1}$.

$\mathrm{H}_{5}$ There exist constants $\sigma_{3}, \sigma_{4}$ such that for every $\mu \in I$ the approximated normal frequencies $\lambda_{\mu}=\left(\lambda_{1, \mu}, \ldots, \lambda_{n-r, \mu}\right)$ satisfy

$$
0<\frac{\sigma_{3}}{2}<\left|\lambda_{i, \mu}\right|<\frac{\sigma_{4}}{2}, \quad 0<\sigma_{3}<\left|\lambda_{i, \mu} \pm \lambda_{j, \mu}\right|
$$

for $i, j=1, \ldots, n-r$, with $i \neq j$.

$\mathrm{H}_{6}$ The objects $h_{\mu}, \tau_{\mu}, N_{\mu}$ and $\lambda_{\mu}$ are at least $\mathcal{C}^{1}$ with respect to $\mu$, and we have

$$
\left\|\frac{d h}{d \mu}\right\|_{I, \mathcal{C}^{3}, \mathcal{U}},\left\|\frac{d \tau}{d \mu}\right\|_{I, \rho},\left\|\frac{d D \tau}{d \mu}\right\|_{I, \rho},\left\|\frac{d N}{d \mu}\right\|_{I, \rho},\left\|\frac{d \lambda_{i}}{d \mu}\right\|_{I}<\sigma_{5},
$$

for $i=1, \ldots, n-r$. Moreover, we have the next separation conditions

$$
0<\frac{\sigma_{6}}{2}<\left|\frac{d}{d \mu} \lambda_{i, \mu}\right|, \quad 0<\sigma_{6}<\left|\frac{d}{d \mu} \lambda_{i, \mu} \pm \frac{d}{d \mu} \lambda_{j, \mu}\right|,
$$

for $i, j=1, \ldots, n-r$, with $i \neq j$.

Under these assumptions, given $\gamma_{0} \leq \frac{1}{2} \min \{1, \hat{\gamma}\}$ and $\nu>\hat{\nu}$, there exists a constant $C_{1}$, that depends on the initial objects but is independent of $\gamma_{0}$, such that if

$$
\varepsilon_{*}=\|e\|_{I, \rho}+\|R\|_{I, \rho}+\left\|\frac{d e}{d \mu}\right\|_{I, \rho}+\left\|\frac{d R}{d \mu}\right\|_{I, \rho}
$$

satisfies $\varepsilon_{*} \leq C_{1} \gamma_{0}^{8}$, then there exists a Cantorian subset $I_{(\infty)} \subset I$ such that $\forall \mu \in I_{(\infty)}$ the Hamiltonian $h_{\mu}$ has an r-dimensional elliptic invariant torus $\mathscr{T}_{\mu,(\infty)}$ with basic frequencies $\omega$ and normal frequencies $\lambda_{\mu,(\infty)}$ that satisfy Diophantine conditions of the form

$$
\left|\langle k, \omega\rangle+\lambda_{i, \mu,(\infty)}\right| \geq \frac{\gamma_{0}}{|k|_{1}^{\nu}}, \quad\left|\langle k, \omega\rangle+\lambda_{i, \mu,(\infty)} \pm \lambda_{j, \mu,(\infty)}\right| \geq \frac{\gamma_{0}}{|k|_{1}^{\nu}}, \quad \forall k \in \mathbb{Z}^{r} \backslash\{0\}
$$


for $i, j=1, \ldots, n-r$, such that

$$
\left\|\tau_{(\infty)}-\tau\right\|_{I_{(\infty)}, \rho / 2} \leq \frac{C_{2} \varepsilon_{*}}{\gamma_{0}^{2}}, \quad\left\|N_{(\infty)}-N\right\|_{I_{(\infty)}, \rho / 2} \leq \frac{C_{2} \varepsilon_{*}}{\gamma_{0}^{4}},
$$

and for $i=1, \ldots, n-r$,

$$
\left\|\lambda_{i,(\infty)}-\lambda_{i}\right\|_{I_{(\infty)}} \leq \frac{C_{2} \varepsilon_{*}}{\gamma_{0}^{2}}
$$

Moreover, $I_{(\infty)}$ has big relative Lebesgue measure

$$
\operatorname{meas}_{\mathbb{R}}\left(I \backslash I_{(\infty)}\right) \leq C_{3} \gamma_{0} .
$$

The constants $C_{2}$ and $C_{3}$ depend on $|\omega|, \hat{\gamma}, \hat{\nu}, \nu, r, n, \sigma_{0}, \sigma_{1}, \sigma_{2}, \sigma_{3}, \sigma_{4}, \sigma_{5}$ and $\sigma_{6}$.

Remark 3.2. We will see that, if $\|e\|_{I, \rho}$ and $\|R\|_{I, \rho}$ are small enough, hypothesis $\mathrm{H}_{2}$ and $\mathrm{H}_{5}$, together with suitable Diophantine conditions on $\omega$ and $\lambda$, imply that the matrix $\Omega_{N, N^{*}}$ is pure imaginary, approximately constant and close to diagonal (see Propositions 4.1 and 5.3 for details). In order to follow our approach for constructing an approximately symplectic basis along the torus, we assume that the average of this matrix is non-singular. According to this, it is clear that we can assume (after a suitable choice of the sign of the components of $\lambda$ and scaling of the columns of $N$ ) that diag $\left[\Omega_{N, N^{*}}\right]_{\mathbb{T}^{r}}=\mathrm{iId}_{n-r}$, as it is done in hypothesis $\mathrm{H}_{3}$ of Theorem 3.1.

Remark 3.3. As it is customary in parameterization methods -we encourage the reader to compare this result with those in [13,20,31]-the conditions of Theorem 3.1 can be verified using information provided by the initial approximations. This fact is useful in the validation of numerical computations that consist in looking for trigonometric functions that satisfy invariance and reducibility equations approximately. Concretely, let us assume that for a given parameter $\mu_{0} \in I$ we have computed approximations $\tau_{\mu_{0}}, N_{\mu_{0}}$ and $\Lambda_{\mu_{0}}$ satisfying the explicit conditions of Theorem 3.1 for certain $\omega \in \mathbb{R}^{r}$. Then, for most of the values of $\mu$ close to $\mu_{0}$, there exist an elliptic quasi-periodic invariant torus nearby, whose normal frequencies are just slightly changed.

Remark 3.4. Hypothesis $\mathrm{H}_{4}$ is called twist condition because when applying this result in a perturbative setting it stands for the Kolmogorov non-degeneracy condition (see the computations performed for Hamiltonian (13) below). Observe that in the Lagrangian case hypothesis $A_{1, \mu}$ reads as $A_{1, \mu}=G_{D \tau_{\mu}}^{-1} D \tau_{\mu}^{\top} T_{1, \mu} D \tau G_{D \tau_{\mu}}^{-1}$ for the same matrix $T_{1, \mu}$, thus recovering the condition in [13].

Remark 3.5. Let us assume that for $\mu=0$ we have a true elliptic quasi-periodic invariant torus satisfying the Diophantine and non-degeneracy conditions of Theorem 3.1. In this case, it is expected that the measure of true invariant tori nearby is larger that the one predicted by our result. Actually, it is known that the complementary set $\left[-\mu_{0}, \mu_{0}\right] \backslash I_{(\infty)}$ has measure 
exponentially small when $\mu_{0} \rightarrow 0$ (see $\left.[35,36]\right)$. To obtain such estimates we would need to modify slightly some details of the proof performed here - but not the scheme- asking for Diophantine conditions as those used in [34, 36] (which turn out to be exponentially small in $\left.|k|_{1}\right)$.

Remark 3.6. Theorem 3.1 can be extended to the case of exact symplectic maps. Actually, the parameterization approach in the context of maps is the main setting in [13, 20, 31]. To this end, we should "translate" the computations performed along the paper to the context of maps, following the "dictionary" of these references. Attemption should be taken in order to adapt the geometric conditions that we highlight in Remarks 4.5 and 4.7, which are not true for maps, but satisfied up to quadratic terms (this is enough for the convergence of the scheme).

Remark 3.7. It would be also interesting to extend the result in order to deal with symplectic vector fields or symplectic maps. Let us recall that a vector field $X$ on a symplectic manifold with 2-form $\Omega$ is said to be symplectic if $L_{X} \Omega=0$, i.e., if the 2-form is preserved along the flow of $X$ (symplectic vector fields that are not Hamiltonian can be found for example in the context of magnetic fields). In this situation, the method of "translated torus" should be adapted as it is done in [20] for the hyperbolic case. To this end, it must be taken into account that the cohomology of the torus must be compatible with the cohomology class of the contraction $\Omega(\cdot, X)$.

Remark 3.8. The scheme of the proof of Theorem 3.1 can be also used for proving the existence of reducible tori having some hyperbolic directions, under the assumption of first and second order Melnikov. In this case, we need to adapt the geometrical ideas of the paper in order to deal simultaneously with elliptic and hyperbolic directions. However, as hyperbolic tori are known to exist beyond the breakdown of reducibility (see [25]), it is interesting to approach the problem of partially elliptic tori by combining techniques in [20] (for studying hyperbolic directions) together with those presented here (for studying elliptic directions).

Remark 3.9. The scheme can be also adapted to deal with the classical Broer-Huitema-Takens approach (see [7]) explained in the introduction. On the one hand, this allows obtaining $\mathcal{C}^{\infty}$ Whitney regularity for the constructed tori, and on the other hand this permits to deal with degenerate cases where Kolmogorov condition does not hold, but we have other higher-order nondegeneracy conditions such as the so-called Rüssmann's non-degeneracy condition (see [62]).

Remark 3.10. After the work in $[3,4,19,26,66]$ it is known that second order Melnikov conditions are not necessary for proving existence of lower dimensional tori in the elliptic context. For example, Bourgain approached the problem without using reducibility, thus avoiding to ask for these non-resonance conditions. However, cumbersome multiescale analysis is required to approximate the solution of truncated cohomological equations, thus leading to a process which is not suitable for numerical implementations - at each step, one has to invert a large matrix which has a huge computational cost. Nevertheless, asking for reducibility we end up inverting a diagonal matrix in Fourier space (see Remark 3.11). Another approach to avoid 
second Melnikov conditions was proposed by Eliasson in [19] and consists in performing a far-from-identity transformation when we have to deal with such resonant frequencies. Concretely, if $\lambda \in \mathbb{R}^{n-r}$ does not satisfies second Melnikov conditions, then we can introduce new normal frequencies $\tilde{\lambda}_{j}=\lambda_{j}-\left\langle m_{j}, \omega / 2\right\rangle$, and we can choose carefully the vectors $m_{j} \in \mathbb{Z}^{r}$ in such a way that second Melnikov conditions are satisfied (it is also necessary to work in the double covering $2 \mathbb{T}^{r}=\mathbb{R}^{r} /(4 \pi \mathbb{Z})^{r}$ of the torus). In this paper we study reducible tori without using Eliasson's method (thus emphasizing the geometric ideas linked with parameterization methods), so we ask for second Melnikov conditions paying the price of excluding a small set of invariant tori. Nevertheless, when implementing numerically this method, the use of Elliasson's transformation is very useful (this was used in [25] to continue elliptic tori beyond their bifurcation to hyperbolic tori).

Remark 3.11. All the computations performed in the proof of Theorem 3.1 can be implemented very efficiently in a computer. For example, the solution of cohomological equations with constant coefficients and the computation of derivatives like $D \tau$ or $L_{\omega} \tau$ correspond to diagonal operators in Fourier space. Other algebraic manipulations can be performed efficiently in real space and there are very fast and robust FFT algorithms that allow passing from real (or complex) space to Fourier space (and "vice versa"). Accordingly, if we approximate a torus by using $N$ Fourier modes, we can implement an algorithm to compute the object with a cost of $\operatorname{order} \mathcal{O}(N \log N)$ in time and $\mathcal{O}(N)$ in memory. We refer to the works [9, 14, 32] to analogous algorithms in several contexts. Therefore, this approach presents significant advantages in contrast with methods which require to deal with large matrices, since they represent a cost of $\mathcal{O}\left(N^{2}\right)$ in memory and $\mathcal{O}\left(N^{3}\right)$ in time (we refer for example to [10]).

Although one of the main features of both the formulation and the proof of Theorem 3.1 is that we do not require to write the problem in action-angle coordinates, we think that it can be illustrative to express this result for a close-to-integrable system, in order to clarify the meaning of hypotheses $\mathrm{H}_{3}$ and $\mathrm{H}_{4}$ in this context. Indeed, let us consider the following family of Hamiltonian systems written in action-angle-like coordinates $(\varphi, y, z) \in \mathbb{T}^{r} \times \mathbb{R}^{r} \times \mathbb{R}^{2(n-r)}$

$$
h_{\mu}(\varphi, y, z)=h_{0}(y, z)+\mu f(\varphi, y, z)
$$

such that for $y=0$, we have that $z=0$ is an elliptic non-degenerate equilibrium for the system $h_{0}(y, z)$. This means that $\tau_{0}(\theta)=(\theta, 0,0)$ gives a parameterization of an invariant torus of $h_{0}$ with basic frequencies $\omega=\operatorname{grad}_{y} h_{0}(0,0) \in \mathbb{R}^{r}$. By performing a suitable canonical change of variables in order to eliminate crossed quadratic terms in $(y, z)$, we can assume that

$$
h_{0}(y, z)=\langle\omega, y\rangle+\frac{1}{2}\langle y, A y\rangle+\frac{1}{2}\langle z, B z\rangle+\mathcal{O}_{3}(y, z)
$$

close to $(y, z)=(0,0)$, where $A$ and $B$ are symmetric matrices, such that

$$
\operatorname{spec}\left(J_{n-r} B\right)=\left\{\mathrm{i} \lambda_{1}, \ldots, \mathrm{i} \lambda_{n-r},-\mathrm{i} \lambda_{1}, \ldots,-\mathrm{i} \lambda_{n-r}\right\},
$$


are the normal eigenvalues of the torus given by $\mathbb{T}^{r} \times\{0\} \times\{0\}$. The associated normal directions are given by the real and imaginary parts of the matrix of eigenvectors satisfying $J_{n-r} B \hat{N}=\hat{N} \Lambda$, where $\Lambda=\operatorname{diag}(\mathrm{i} \lambda)=\operatorname{diag}\left(\mathrm{i} \lambda_{1}, \ldots, \mathrm{i} \lambda_{n-r}\right)$. Using symplectic properties, we can select the signs of the components of $\lambda$ and the complex matrix $\hat{N}$ in such a way that it satisfies $\hat{N}^{\top} J_{n-r} \hat{N}^{*}=\mathrm{i} \operatorname{Id}_{n-r}$.

Then, to apply Theorem 3.1 to the family of Hamiltonians $h_{\mu}$ given by (13), for small $|\mu|$, we consider the family of approximately elliptic and invariant tori $\tau_{\mu}(\theta)=\tau_{0}(\theta)+\mathcal{O}(\mu)$ with normal frequencies $\lambda_{\mu}=\lambda+\mathcal{O}(\mu)$ and normal vectors $N_{\mu}(\theta)^{\top}=\left(\begin{array}{ll}0 & 0 \hat{N}^{\top}\end{array}\right)+\mathcal{O}(\mu)$, where the terms $\mathcal{O}(\mu)$ stand for the first order corrections in $\mu$-they can be computed by means of Lindstedt series or normal forms with respect to $\mu$ - that are needed in order to check that the normal frequencies "move" as a function of $\mu$. This family satisfies

$$
\begin{aligned}
L_{\omega} \tau_{\mu}(\theta) & =J \operatorname{grad} h_{\mu}\left(\tau_{\mu}(\theta)\right)+\mathcal{O}_{2}(\mu), \\
L_{\omega} N_{\mu}(\theta) & =J \operatorname{hess} h_{\mu}\left(\tau_{\mu}(\theta)\right) N_{\mu}(\theta)-N_{\mu}(\theta) \Lambda_{\mu}+\mathcal{O}_{2}(\mu),
\end{aligned}
$$

and, for $\mu=0$, we have

$$
D \tau_{0}(\theta)=\left(\begin{array}{c}
\operatorname{Id}_{r} \\
0 \\
0
\end{array}\right), \quad N_{0}(\theta)=\left(\begin{array}{c}
0 \\
0 \\
\hat{N}
\end{array}\right), \quad G_{D \tau_{0}}^{-1}(\theta)=\operatorname{Id}_{r}, \quad \Omega_{N_{0}, N_{0}^{*}}(\theta)=\mathrm{iId}_{n-r} .
$$

Moreover, it is not difficult to check that the matrix $A_{1, \mu}(\theta)$ in $\mathrm{H}_{4}$ at $\mu=0$ reads as $A_{1,0}(\theta)=$ $-A$, which implies that $\mathrm{H}_{4}$ is equivalent to the standard (Kolmogorov) non-degeneracy condition for the unperturbed system.

\section{Overview and heuristics of the method}

In this section we outline the main ideas of the presented approach emphasizing the geometric interpretation of our construction and highlighting the additional difficulties with respect to the Lagrangian and normally hyperbolic cases. First, in Section 4.1, we sketch briefly the proof of Theorem 3.1. Our aim is to emphasize that - even though some parts of the proof involve quite cumbersome computations - the construction of the iterative procedure is fairly natural. Then, in Section 4.2 we focus on the geometric properties of the invariant and elliptic case that allow us to obtain approximate solutions for the equations derived in Section 4.1 associated to approximately invariant and elliptic tori.

\subsection{Sketch of the proof}

Let $h: U \subset \mathbb{R}^{2 n} \rightarrow \mathbb{R}$ be a Hamiltonian function and let us suppose that $\mathscr{T}$ is an approximately invariant and elliptic torus of basic frequencies $\omega \in \mathbb{R}^{r}$ and normal ones $\lambda \in \mathbb{R}^{n-r}$, satisfying 
non-resonance conditions (4) and (9). The translation of Definitions 2.5 and 2.9 into a functional setting is

$$
\mathcal{F}(\tau)=e, \quad \mathcal{G}(\tau, N, \Lambda)=R,
$$

with $\Lambda=\operatorname{diag}(\mathrm{i} \lambda)$, where we have introduced the following operators

$$
\begin{aligned}
\mathcal{F}(\tau) & =L_{\omega} \tau-\operatorname{Jgrad} h(\tau), \\
\mathcal{G}(\tau, N, \Lambda) & =L_{\omega} N-J \operatorname{hess} h(\tau) N+N \Lambda .
\end{aligned}
$$

Then, we look for an embedding $\bar{\tau}: \mathbb{T}^{r} \rightarrow U$ and a set of normal vectors $\bar{N}: \mathbb{T}^{r} \rightarrow$ $M_{2 n \times(n-r)}(\mathbb{C})$, with normal frequencies $\bar{\lambda}$, satisfying

$$
\mathcal{F}(\bar{\tau})=0, \quad \mathcal{G}(\bar{\tau}, \bar{N}, \bar{\Lambda})=0,
$$

with $\bar{\Lambda}=\operatorname{diag}(\mathrm{i} \bar{\lambda})$. Since these equations have triangular structure, we approach first the correction of the parameterization of the torus, i.e., we look for $\bar{\tau}=\tau+\Delta_{\tau}$ satisfying the above expressions. We write the first equation as

$$
\mathcal{F}\left(\tau+\Delta_{\tau}\right)=e+L_{\omega} \Delta_{\tau}-\operatorname{Jhess} h(\tau) \Delta_{\tau}+O_{2}\left(\Delta_{\tau}\right)=0
$$

If we neglect terms $O_{2}\left(\Delta_{\tau}\right)$ we obtain the following linearized equation (Newton method)

$$
L_{\omega} \Delta_{\tau}-J \operatorname{hess} h(\tau) \Delta_{\tau}=-e,
$$

that allows us to correct the invariance of the torus up to terms of second order in $e$. In a similar way, we look for $\bar{N}=N+\Delta_{N}$ and $\bar{\Lambda}=\Lambda+\Delta_{\Lambda}$ such that

$$
\mathcal{G}(\bar{\tau}, \bar{N}, \bar{\Lambda})=\hat{R}+L_{\omega} \Delta_{N}-J \operatorname{hess} h(\tau) \Delta_{N}+N \Delta_{\Lambda}+\Delta_{N} \Lambda+O_{2}\left(\Delta_{N}, \Delta_{\Lambda}\right)=0,
$$

where

$$
\hat{R}=R+\operatorname{Jhess} h(\tau) N-\operatorname{Jhess} h(\bar{\tau}) N
$$

includes both the error in reducibility and the one introduced when correcting the torus (which is expected to be of order of the size of $e$ ). Hence, in order to apply one step of the Newton method to correct reducibility, we have to solve the following linearized equation for $\Delta_{N}$ and $\Delta_{\Lambda}$

$$
L_{\omega} \Delta_{N}-\operatorname{Jhess} h(\tau) \Delta_{N}+N \Delta_{\Lambda}+\Delta_{N} \Lambda=-\hat{R} .
$$

For convenience, once we fix $\tau, N$ and $\Lambda$, we define the following differential operators (acting on vectors or matrices of $2 n$ rows)

$$
\begin{aligned}
\mathcal{R}(\xi) & =L_{\omega} \xi-J \operatorname{hess} h(\tau) \xi, \\
\mathcal{S}(\xi, \eta) & =\mathcal{R}(\xi)+N \eta+\xi \Lambda,
\end{aligned}
$$


so Equations (14) and (16) are equivalent to invert $\mathcal{R}$ and $\mathcal{S}$

$$
\mathcal{R}\left(\Delta_{\tau}\right)=-e, \quad \mathcal{S}\left(\Delta_{N}, \Delta_{\Lambda}\right)=-\hat{R}
$$

As it was done in [13], the main idea is to use the geometric properties of the problem to prove that the linearized Equation (14) can be transformed, using a suitable basis along the approximate torus, into a simpler linear equation - with constant coefficients - that can be approximately solved by means of Fourier series. Indeed, an approximate solution with an error of quadratic size in $e$ and $R$ is enough for the convergence of the scheme - the Newton method still converges quadratically if we have a good enough approximation of the Jacobian matrix. Under suitable conditions of non-resonance and non-degeneracy, iteration of this process leads to a quadratic scheme that allows us to overcome the effect of the small divisors of the problem. The main contribution of this paper is to adapt this construction (that we describe next in a more precise way) to deal with Equations (14) and (16) simultaneously.

Let us discuss the construction of the basis mentioned above. In the Lagrangian case we only have to deal with Equation (14) and the columns of the matrices $D \tau$ and $J D \tau G_{D \tau}^{-1}$ give us an approximately symplectic basis of $\mathbb{R}^{2 n}$ at any point of the torus. Moreover, it turns out that $\mathcal{R}(D \tau)=0+\mathcal{O}(e)$ and $\mathcal{R}\left(J D \tau G_{D \tau}^{-1}\right)=D \tau A_{1}+\mathcal{O}(e)$, where $A_{1}: \mathbb{T}^{n} \rightarrow M_{n \times n}(\mathbb{R})$ is a symmetric matrix. Using this basis we can write the linearized equation (14) in "triangular form" with respect to the projections of $\Delta_{\tau}$ over $D \tau$ and $J D \tau G_{D \tau}^{-1}$, in such a way that the problem is reduced to solve two cohomological equations with constant coefficients. However, in the lower dimensional case the previous construction is not enough since we also have to take into account the normal directions of the torus. As mentioned in the introduction, this scheme has been recently adapted in [20] for the normally hyperbolic case, without requiring reducibility of the normal variational equations. The main ingredient is that there exists a splitting between the center and the hyperbolic directions of the torus and we can reduce the study of Equation (14) to the projections according to this splitting. The dynamics on the hyperbolic directions is characterized by asymptotic (geometric) growth conditions ${ }^{2}$ — both in the future and in the pastand the linearized equation (14) restricted to the center subspace follows as in the Lagragian case (now the ambient space is $\mathbb{R}^{2 r}$ ).

In the normally elliptic context, we ask for reducibility in order to express equation (14) in a simple form. Hence, we solve simultaneously equation (16), thus obtaining a basis that reduces the normal variational equations of the torus to constants coefficients up to a quadratic error. In this case, the approximately (with an error of the order of the size of $e$ and $R$ ) symplectic basis is obtained by completing the columns of $D \tau, N$ and i $N^{*}$ with the columns of a suitably constructed matrix $V: \mathbb{T}^{r} \rightarrow M_{2 n \times r}(\mathbb{R})$. Basically, we take advantage of the fact that $V$ satisfies $\mathcal{R}(V)=D \tau A_{1}$ modulo terms of order $e$ and $R$, where $A_{1}: \mathbb{T}^{r} \rightarrow M_{r \times r}(\mathbb{R})$ will be specified later on. Hence, we find approximately solutions for equations (19) in terms of the

\footnotetext{
${ }^{2}$ Concretely, the solution for the equations projected into the hyperbolic directions are obtained by means of absolutely convergent power series. See details in [20].
} 
constructed basis as follows

$$
\begin{aligned}
\Delta_{\tau} & =D \tau \Delta_{1}+V \Delta_{2}+N \Delta_{3}+\mathrm{i} N^{*} \Delta_{4}, \\
\Delta_{N} & =D \tau P_{1}+V P_{2}+N P_{3}+\mathrm{i} N^{*} P_{4},
\end{aligned}
$$

where $\left\{\Delta_{i}\right\},\left\{P_{i}\right\}$, with $i=1, \ldots, 4$, are the solutions of cohomological equations (37)-(40), and (42)-(45), respectively. The correction $\Delta_{\Lambda}$ in the normal eigenvalues is determined from the compatibility condition of these last equations.

Let us observe that in order to correct the reducibility of the torus we have to change slightly the normal directions and the normal frequencies. Since the normal frequencies $\lambda$ are modified at each step of the process, we do not know in advance if they will satisfy the required Diophantine conditions for all steps - unless we have enough parameters to control the value of all of them simultaneously. To deal with this problem we require some control on the change of these frequencies, in such a way that we can remove parameters that give rise to resonant frequencies. Since at every step of the inductive process we are removing a dense set of parameters, this does not allow us to keep any kind of smooth dependence with respect to them (because now they move on a set of empty interior).

There are several methods in the literature to deal with this problem. The first approach was due to Arnold (see [1]) and it consists in working, at every step of the inductive procedure, with a finite number of terms in the Fourier expansions ("ultraviolet cut-off"). Then, since we only need to deal with a finite number of resonances at every step, we can work on open sets of parameters and keep the smooth dependence on these sets. Another possibility is to consider Lipschitz parametric dependence and to check that this dependence is preserved along the iterative procedure (this is the method used in $[33,34,35,36]$ ). Lipschitz regularity suffices to control the measure of the resonant sets. In this paper we follow the Lipschitz approach because it does not forces to modify, by the effect of the "ultraviolet cut-off", the geometric construction we have developed in the Diophantine case.

\subsection{Characterization of the invariant and reducible case}

Our goal now is to formally "invert" the linear operators $\mathcal{R}$ given by (17) and $\mathcal{S}$ given by (18) — see Propositions 4.4 and 4.6, respectively — when the corresponding torus $\mathscr{T}$ is invariant and normally elliptic. In order to do this, first we characterize at a formal level some geometric properties of lower dimensional elliptic invariant tori. Later on, the same construction provided in this section will be used to study approximately invariant tori in order to solve equations in (19) with a small error (controlled by the errors of invariance and reducibility).

All along this section we consider an $r$-dimensional normally elliptic quasi-periodic invariant torus $\mathscr{T}$ for a Hamiltonian $h$, of basic frequencies $\omega \in \mathbb{R}^{r}$ and normal frequencies $\lambda \in \mathbb{R}^{n-r}$ satisfying non-resonance conditions (2), (7) and (8), i.e., we have

$$
\begin{aligned}
L_{\omega} \tau(\theta) & =J \operatorname{grad} h(\tau(\theta)), \\
L_{\omega} N(\theta) & =J \operatorname{hess} h(\tau(\theta)) N(\theta)-N(\theta) \Lambda,
\end{aligned}
$$


with $\Lambda=\operatorname{diag}(\mathrm{i} \lambda)$. We assume also that the matrices $G_{D \tau}(\theta)$ and $G_{N, N^{*}}(\theta)$ are invertible for every $\theta \in \mathbb{T}^{r}$. Then, we claim (see the proof if Proposition 4.1) that under these conditions $\Omega_{N, N^{*}}$ is constant, pure imaginary and diagonal. If we assume that this matrix is non-singular, then we can suppose that (see Remark 3.2)

$$
\Omega_{N, N^{*}}(\theta)=\operatorname{iId}_{n-r} .
$$

Proposition 4.1. Given $\mathscr{T}$ an invariant and elliptic torus as above, we define the matrix functions

$$
N_{1}(\theta)=N(\theta), \quad N_{2}(\theta)=\mathrm{i} N^{*}(\theta)
$$

and the real matrix

$$
V(\theta)=J D \tau(\theta) G_{D \tau}^{-1}(\theta)+N_{1}(\theta) B_{1}(\theta)+N_{2}(\theta) B_{2}(\theta)+D \tau(\theta) B_{3}(\theta),
$$

where

$$
\begin{aligned}
& B_{1}(\theta)=G_{N_{2}, D \tau}(\theta) G_{D \tau}^{-1}(\theta), \\
& B_{2}(\theta)=-G_{N_{1}, D \tau}(\theta) G_{D \tau}^{-1}(\theta), \\
& B_{3}(\theta)=\operatorname{Re}\left(G_{B_{2}, B_{1}}(\theta)\right)
\end{aligned}
$$

Then, the columns of the matrices $D \tau(\theta), V(\theta), N_{1}(\theta)$ and $N_{2}(\theta)$ form a symplectic basis for any $\theta \in \mathbb{T}^{r}$, in the sense that the matrices $\Omega_{D \tau}(\theta), \Omega_{V}(\theta), \Omega_{N_{i}}(\theta), \Omega_{D \tau, N_{i}}(\theta)$ and $\Omega_{N_{i}, V}(\theta)$ vanish, for $i=1,2$, and

$$
\Omega_{N_{2}, N_{1}}(\theta)=\operatorname{Id}_{n-r}, \quad \Omega_{V, D \tau}(\theta)=\operatorname{Id}_{r}
$$

Proof. To obtain the geometric properties associated to the matrices $D \tau, N_{1}$ and $N_{2}$ we proceed as in the proof of Lemma 2.3, where we proved that $\Omega_{D \tau}=0$. Let us start studying the matrix $\Omega_{N_{2}, N_{1}}$ by computing

$$
\begin{aligned}
L_{\omega} \Omega_{N_{2}, N_{1}} & =L_{\omega}\left(N_{2}^{\top} J N_{1}\right)=\left(L_{\omega} N_{2}\right)^{\top} J N_{1}+N_{2}^{\top} J L_{\omega} N_{1} \\
& =\left(J \operatorname{hess} h(\tau) N_{2}+N_{2} \Lambda\right)^{\top} J N_{1}+N_{2}^{\top} J\left(J \operatorname{hess} h(\tau) N_{1}-N_{1} \Lambda\right) \\
& =\Lambda N_{2}^{\top} J N_{1}-N_{2}^{\top} J N_{1} \Lambda=\Lambda \Omega_{N_{2}, N_{1}}-\Omega_{N_{2}, N_{1}} \Lambda .
\end{aligned}
$$

Then, if we expand $\Omega_{N_{2}, N_{1}}$ in Fourier series we obtain

$$
\left(\langle k, \omega\rangle-\lambda_{i}+\lambda_{j}\right)\left(\widehat{\Omega}_{N_{2}, N_{1}}\right)_{k}^{(i, j)}=0
$$

where $\left(\Omega_{N_{2}, N_{1}}\right)^{(i, j)}$ denotes the $(i, j)$-th entry of $\Omega_{N_{2}, N_{1}}$. Recalling the non-resonance hypothesis $\langle k, \omega\rangle-\lambda_{i}+\lambda_{j} \neq 0$ (if $i \neq j$ or $k \neq 0$ ) we obtain that $\left(\widehat{\Omega}_{N_{2}, N_{1}}\right)_{k}^{(i, j)}=0$, for all $k \in \mathbb{Z}^{r} \backslash\{0\}$, and $\left(\widehat{\Omega}_{N_{2}, N_{1}}\right)_{0}^{(i, j)}=0$ if $i \neq j$, so this matrix is constant and diagonal. 
Moreover, $\Omega_{N_{2}, N_{1}}^{\top}=\Omega_{N_{2}, N_{1}}^{*}$ so its entries are real. Finally, using hypothesis (22) we write $\Omega_{N_{2}, N_{1}}=\mathrm{i} \Omega_{N^{*}, N}=-\mathrm{i} \Omega_{N, N^{*}}^{\top}=\operatorname{Id}_{n-r}$.

To prove that $\Omega_{N_{1}}$ vanishes we compute

$$
L_{\omega} \Omega_{N_{1}}=-\Lambda \Omega_{N_{1}}-\Omega_{N_{1}} \Lambda
$$

in a similar way as above. Now, the Fourier coefficients of $\Omega_{N_{1}}$ satisfy

$$
\left(\langle k, \omega\rangle+\lambda_{i}+\lambda_{j}\right)\left(\widehat{\Omega}_{N_{1}}\right)_{k}^{(i, j)}=0
$$

so it turns out that all of them vanish (using the non-resonance conditions). Moreover, taking derivatives at Equation (20) we obtain

$$
L_{\omega} D \tau=J \operatorname{hess} h D \tau
$$

that together with Equation (21), leads to

$$
L_{\omega} \Omega_{D \tau, N_{1}}=-\Omega_{D \tau, N_{1}} \Lambda
$$

which implies that $\Omega_{D \tau, N_{1}}=0$, since the Fourier coefficients of the component functions satisfy the equation

$$
\left(\langle k, \omega\rangle+\lambda_{i}\right)\left(\widehat{\Omega}_{D \tau, N_{1}}\right)_{k}^{(i, j)}=0 .
$$

Finally, it is easy to see that $\Omega_{N_{2}}=-\Omega_{N_{1}}^{*}$ and $\Omega_{D \tau, N_{2}}=\mathrm{i} \Omega_{D \tau, N_{1}}^{*}$, so these matrices also vanish.

Next, we see that the columns of the (real) matrices $D \tau, J D \tau G_{D \tau}^{-1}, \operatorname{Re}(N)$ and $\operatorname{Im}(N)$ form a $\mathbb{R}$-basis of $\mathbb{R}^{2 n}$. To this end, it suffices to check that the columns of $D \tau, J D \tau G_{D \tau}^{-1}, N_{1}$ and $N_{2}$ are $\mathbb{C}$-independent on $\mathbb{C}^{2 n}$. Thus, let us consider a linear combination

$$
D \tau a+J D \tau G_{D \tau}^{-1} b+N_{1} c+N_{2} d=0,
$$

for vector functions $a, b: \mathbb{T}^{r} \rightarrow \mathbb{C}^{r}$ and $c, d: \mathbb{T}^{r} \rightarrow \mathbb{C}^{n-r}$. Multiplying by $D \tau^{\top}, D \tau^{\top} J, N_{2}^{\top} J$ and $N_{1}^{\top} J$ and using the geometric properties proved above, we obtain the following system of equations

$$
\underbrace{\left(\begin{array}{cccc}
G_{D \tau} & 0 & G_{D \tau, N_{1}} & G_{D \tau, N_{2}} \\
0 & -\mathrm{Id}_{r} & 0 & 0 \\
0 & -G_{N_{2}, D \tau} G_{D \tau}^{-1} & \operatorname{Id}_{n-r} & 0 \\
0 & -G_{N_{1}, D \tau} G_{D \tau}^{-1} & 0 & -\operatorname{Id}_{n-r}
\end{array}\right)}_{M_{1}}\left(\begin{array}{l}
a \\
b \\
c \\
d
\end{array}\right)=\left(\begin{array}{l}
0 \\
0 \\
0 \\
0
\end{array}\right),
$$

where $\operatorname{det} M_{1}=\operatorname{det} G_{D \tau} \neq 0$, so we conclude that $a=b=0$ and $c=d=0$.

To check that the matrix $V$ is real, we use the expressions $N_{1}^{*}=-\mathrm{i} N_{2}$ and $B_{1}^{*}=\mathrm{i} B_{2}$ that are obtained in a straightforward way. Then, we compute $N_{1}^{*} B_{1}^{*}=-\mathrm{i}^{2} N_{2} B_{2}=N_{2} B_{2}$, thus concluding that $V^{*}=V$. 
Finally, the following computations are straightforward

$$
\begin{aligned}
\Omega_{D \tau, V} & =-\mathrm{Id}_{r}+\Omega_{D \tau, N_{1}} B_{1}+\Omega_{D \tau, N_{2}} B_{2}+\Omega_{D \tau} B_{3}=-\mathrm{Id}_{r}, \\
\Omega_{N_{1}, V} & =-G_{N_{1}, D \tau} G_{D \tau}^{-1}+\Omega_{N_{1}} B_{1}+\Omega_{N_{1}, N_{2}} B_{2}+\Omega_{N_{1}, D \tau} B_{3} \\
& =-G_{N_{1}, D \tau} G_{D \tau}^{-1}-B_{2}=0, \\
\Omega_{N_{2}, V} & =-G_{N_{2}, D \tau} G_{D \tau}^{-1}+B_{1}=0, \\
\Omega_{V} & =\left(G_{D \tau}^{-1} D \tau^{\top} J^{\top}+B_{1}^{\top} N_{1}^{\top}+B_{2}^{\top} N_{2}^{\top}+B_{3}^{\top} D \tau^{\top}\right) J V \\
& =G_{D \tau}^{-1} G_{D \tau, N_{1}} B_{1}+G_{D \tau}^{-1} G_{N_{2}, D \tau} B_{2}+B_{3}-B_{3}^{\top} \\
& =-G_{B_{2}, B_{1}}+G_{B_{1}, B_{2}}+B_{3}-B_{3}^{\top} \\
& =\operatorname{iIm}\left(G_{B_{1}, B_{2}}-G_{B_{2}, B_{1}}\right)=0 .
\end{aligned}
$$

In the last computation we used that $V$ is real.

Remark 4.2. Notice that the matrix $B_{3}$ can be taken modulo the addition of a symmetric real matrix. This freedom can be used to ask for reducibility also in the "central directions" of the torus. Hence, instead of the matrix $A_{1}$ that appears in Lemma 4.3 we would obtain its average $\left[A_{1}\right]_{\mathbb{T}^{r}}$. Since this does not give us any significant advantage, we do not resort to this fact.

In the invariant and reducible case, we characterize the action of $\mathcal{R}$ on $D \tau(\theta), N_{1}(\theta)$ and $N_{2}(\theta)$ in a very simple way

$$
\mathcal{R}(D \tau(\theta))=0, \quad \mathcal{R}\left(N_{1}(\theta)\right)=-N_{1}(\theta) \Lambda, \quad \mathcal{R}\left(N_{2}(\theta)\right)=N_{2}(\theta) \Lambda .
$$

The first expression follows immediately from equation (20) —invariance — and the other ones from equation (21) —reducibility. Moreover, we have the following result for $V(\theta)$.

Lemma 4.3. Under the setting of Proposition 4.1, we have that

$$
\mathcal{R}(V(\theta))=D \tau(\theta) A_{1}(\theta)
$$

where $A_{1}: \mathbb{T}^{r} \rightarrow M_{r \times r}(\mathbb{R})$ is given by the real symmetric matrix

$$
A_{1}(\theta)=G_{D \tau}^{-1}(\theta) D \tau(\theta)^{\top}\left(T_{1}(\theta)+T_{2}(\theta)+T_{2}(\theta)^{\top}\right) D \tau(\theta) G_{D \tau}^{-1}(\theta),
$$

where

$$
\begin{aligned}
& T_{1}(\theta)=J^{\top} \text { hess } h(\tau(\theta)) J-\operatorname{hess} h(\tau(\theta)), \\
& T_{2}(\theta)=T_{1} J\left[D \tau(\theta) G_{D \tau}(\theta)^{-1} D \tau(\theta)^{\top}-\mathrm{Id}\right] \operatorname{Re}\left(N_{1}(\theta) N_{2}(\theta)^{\top}\right) .
\end{aligned}
$$


Proof. We only have to write the expression for $\mathcal{R}(V)$ in terms of the previously constructed symplectic basis

$$
\mathcal{R}(V)=D \tau A_{1}+V A_{2}+N_{1} A_{3}+N_{2} A_{4},
$$

and then to show that $A_{1}$ is given by (29) and $A_{2}=A_{3}=A_{4}=0$. First, we use (23) and (28) to express $\mathcal{R}(V)$ as

$$
\mathcal{R}(V)=\mathcal{R}\left(J D \tau G_{D \tau}^{-1}\right)+N_{1}\left(L_{\omega} B_{1}-\Lambda B_{1}\right)+N_{2}\left(L_{\omega} B_{2}+\Lambda B_{2}\right)+D \tau L_{\omega} B_{3} .
$$

Then, multiplying at both sides of equation (31) by $V^{\top} J, D \tau^{\top} J, N_{2}^{\top} J, N_{1}^{\top} J$ and using the symplectic properties of the basis we obtain the following expressions:

$$
\begin{aligned}
& A_{1}=L_{\omega} B_{3}+V^{\top} J \mathcal{R}\left(J D \tau G_{D \tau}^{-1}\right), \\
& A_{2}=-D \tau^{\top} J \mathcal{R}\left(J D \tau G_{D \tau}^{-1}\right), \\
& A_{3}=L_{\omega} B_{1}-\Lambda B_{1}+N_{2}^{\top} J \mathcal{R}\left(J D \tau G_{D \tau}^{-1}\right), \\
& A_{4}=L_{\omega} B_{2}+\Lambda B_{2}-N_{1}^{\top} J \mathcal{R}\left(J D \tau G_{D \tau}^{-1}\right) .
\end{aligned}
$$

First, introducing $B_{1}=G_{N_{2}, D \tau} G_{D \tau}^{-1}$ into equation (34), we obtain

$$
\begin{aligned}
A_{3}= & L_{\omega}\left(N_{2}^{\top} D \tau G_{D \tau}^{-1}\right)-\Lambda N_{2}^{\top} D \tau G_{D \tau}^{-1}+N_{2}^{\top} J \mathcal{R}\left(J D \tau G_{D \tau}^{-1}\right) \\
= & L_{\omega} N_{2}^{\top} D \tau G_{D \tau}^{-1}+N_{2}^{\top} L_{\omega}\left(D \tau G_{D \tau}^{-1}\right)-\Lambda N_{2}^{\top} D \tau G_{D \tau}^{-1} \\
& +N_{2}^{\top} J L_{\omega}\left(J D \tau G_{D \tau}^{-1}\right)+N_{2}^{\top} \operatorname{hess} h J D \tau G_{D \tau}^{-1} \\
= & (\underbrace{L_{\omega} N_{2}-J \operatorname{hess} h N_{2}}_{\mathcal{R}\left(N_{2}\right)}-N_{2} \Lambda)^{\top} D \tau G_{D \tau}^{-1}=0,
\end{aligned}
$$

where we used the property (28) for $N_{2}$. Recalling that $N_{2}=\mathrm{i} N_{1}^{*}$ we observe that $A_{3}^{*}=\mathrm{i} A_{4}$ so we also have $A_{4}=0$.

Now, we expand the expression for $\mathcal{R}\left(J D \tau G_{D \tau}^{-1}\right)$, obtaining

$$
\begin{aligned}
\mathcal{R}\left(J D \tau G_{D \tau}^{-1}\right)= & \mathcal{R}(J D \tau) G_{D \tau}^{-1}+J D \tau L_{\omega}\left(G_{D \tau}^{-1}\right) \\
= & -\operatorname{hess} h D \tau G_{D \tau}^{-1}-J D \tau G_{D \tau}^{-1}\left(D \tau^{\top} J \operatorname{hess} h-D \tau^{\top} \operatorname{hess} h J\right) D \tau G_{D \tau}^{-1} \\
& -J \text { hess } h J D \tau G_{D \tau}^{-1}=\left(\operatorname{Id}_{r}+J D \tau G_{D \tau}^{-1} D \tau^{\top} J\right) T_{1} D \tau G_{D \tau}^{-1},
\end{aligned}
$$

where we used expression (30) for $T_{1}$. Then, on the one hand we have $D \tau^{\top} J \mathcal{R}\left(J D \tau G_{D \tau}^{-1}\right)=0$ - in combination with (33) this implies that $A_{2}=0$ - and on the other hand we have

$$
\begin{aligned}
V^{\top} J \mathcal{R}\left(J D \tau G_{D \tau}^{-1}\right) & =\left(B_{3}^{\top} D \tau^{\top}+B_{2}^{\top} N_{2}^{\top}+B_{1}^{\top} N_{1}^{\top}+G_{D \tau}^{-1} D \tau^{\top} J^{\top}\right) J \mathcal{R}\left(J D \tau G_{D \tau}^{-1}\right) \\
& =-B_{2}^{\top}\left(L_{\omega} B_{1}-\Lambda B_{1}\right)+B_{1}^{\top}\left(L_{\omega} B_{2}+\Lambda B_{2}\right)+G_{D \tau}^{-1} D \tau^{\top} T_{1} D \tau G_{D \tau}^{-1},
\end{aligned}
$$


where we have used equations (34) and (35) taking into account that $A_{3}=A_{4}=0$. Finally, we introduce this last expression into (32) and recall that $B_{3}=\operatorname{Re}\left(G_{B_{2}, B_{1}}\right)$ in order to obtain

$$
\begin{aligned}
A_{1} & =\operatorname{Re}\left(L_{\omega}\left(B_{2}^{\top} B_{1}\right)\right)-B_{2}^{\top}\left(L_{\omega} B_{1}-\Lambda B_{1}\right)+B_{1}^{\top}\left(L_{\omega} B_{2}+\Lambda B_{2}\right)+G_{D \tau}^{-1} D \tau^{\top} T_{1} D \tau G_{D \tau}^{-1} \\
& =\operatorname{Re}\left(L_{\omega} B_{2}^{\top} B_{1}-B_{2}^{\top} L_{\omega} B_{1}+2 B_{2}^{\top} \Lambda B_{1}\right)+G_{D \tau}^{-1} D \tau^{\top} T_{1} D \tau G_{D \tau}^{-1},
\end{aligned}
$$

where we used that $\left(B_{1}^{\top}\left(L_{\omega} B_{2}+\Lambda B_{2}\right)\right)^{*}=-B_{2}^{\top}\left(L_{\omega} B_{1}-\Lambda B_{1}\right)$. Now we replace $B_{1}$ and $B_{2}$ by equations (24) and (25) respectively, and we expand the expression for $L_{\omega} B_{1}$ and $L_{\omega} B_{2}$ as follows (we also use that $B_{1}=-\mathrm{i} B_{2}^{*}$ )

$$
\begin{aligned}
L_{\omega} B_{2} & =-L_{\omega} N_{1}^{\top} D \tau G_{D \tau}^{-1}-N_{1}^{\top} L_{\omega}\left(D \tau G_{D \tau}^{-1}\right) \\
& =\Lambda N_{1}^{\top} D \tau G_{\tau}^{-1}-N_{1}^{\top} \operatorname{hess} h J^{\top} D \tau G_{D \tau}^{-1}-N_{1}^{\top} L_{\omega}\left(D \tau G_{D \tau}^{-1}\right) \\
& =-\Lambda B_{2}+N_{1}^{\top} J T_{1} D \tau G_{D \tau}^{-1}-N_{1}^{\top} D \tau L_{\omega}\left(G_{D \tau}^{-1}\right) \\
& =-\Lambda B_{2}+N_{1}^{\top} J T_{1} D \tau G_{D \tau}^{-1}-N_{1}^{\top} D \tau G_{D \tau}^{-1} D \tau^{\top} J T_{1} D \tau G_{D \tau}^{-1} . \\
L_{\omega} B_{1} & =\Lambda B_{1}-N_{2}^{\top} J T_{1} D \tau G_{D \tau}^{-1}+N_{2}^{\top} D \tau G_{D \tau}^{-1} D \tau^{\top} J T_{1} D \tau G_{D \tau}^{-1} .
\end{aligned}
$$

From this expressions we observe that term $2 B_{2}^{\top} \Lambda B_{1}$ in $A_{1}$ is cancelled. Finally, since $\left(N_{1} N_{2}^{\top}\right)^{*}=$ $-N_{2} N_{1}^{\top}=\left(-N_{1} N_{2}^{\top}\right)^{\top}$, it turns out that $\operatorname{Re}\left(N_{1} N_{2}^{\top}\right)=\operatorname{Re}\left(\left(-N_{1} N_{2}^{\top}\right)^{\top}\right)$ so we obtain the expression (29) for $A_{1}$.

Now we have all the ingredients for inverting formally the operator $\mathcal{R}$.

Proposition 4.4. Under the setting of Proposition 4.1, we assume that the matrix $A_{1}$ given in (29) satisfies the twist condition $\operatorname{det}\left[A_{1}\right]_{\mathbb{T}^{r}} \neq 0$. Then, given a function $e: \mathbb{T}^{r} \rightarrow \mathbb{R}^{2 n}$ satisfying $\left[D \tau^{\top} J e\right]_{\mathbb{T}^{r}}=0$, we obtain a formal solution for the equation

$$
\mathcal{R}\left(\Delta_{\tau}(\theta)\right)=L_{\omega} \Delta_{\tau}-J \operatorname{hess} h(\tau) \Delta_{\tau}=-e(\theta),
$$

which is unique up to terms in $\operatorname{ker}(\mathcal{R})=\left\{D \tau A: A \in M_{r \times r}(\mathbb{R})\right\}$.

Proof. We express the unknown $\Delta_{\tau}(\theta)$ in terms of the constructed symplectic basis

$$
\Delta_{\tau}=D \tau \Delta_{1}+V \Delta_{2}+N_{1} \Delta_{3}+N_{2} \Delta_{4}
$$

expand $\mathcal{R}\left(\Delta_{\tau}\right)$ and project to compute the functions $\left\{\Delta_{i}\right\}_{i=1, \ldots, 4}$. Concretely, we have

$$
\begin{aligned}
\mathcal{R}\left(\Delta_{\tau}\right)= & \mathcal{R}(D \tau) \Delta_{1}+\mathcal{R}(V) \Delta_{2}+\mathcal{R}\left(N_{1}\right) \Delta_{3}+\mathcal{R}\left(N_{2}\right) \Delta_{4} \\
& +D \tau L_{\omega} \Delta_{1}+V L_{\omega} \Delta_{2}+N_{1} L_{\omega} \Delta_{3}+N_{2} L_{\omega} \Delta_{4} \\
= & D \tau\left(L_{\omega} \Delta_{1}+A_{1} \Delta_{2}\right)+V L_{\omega} \Delta_{2}+N_{1}\left(L_{\omega} \Delta_{3}-\Lambda \Delta_{3}\right)+N_{2}\left(L_{\omega} \Delta_{4}+\Lambda \Delta_{4}\right)
\end{aligned}
$$


Multiplying at both sides of this expression by $V^{\top} J, D \tau^{\top} J, N_{2}^{\top} J$ and $N_{1}^{\top} J$, we obtain the following four cohomological equations:

$$
\begin{aligned}
L_{\omega} \Delta_{1}+A_{1} \Delta_{2} & =-V^{\top} J e \\
L_{\omega} \Delta_{2} & =D \tau^{\top} J e \\
L_{\omega} \Delta_{3}-\Lambda \Delta_{3} & =-N_{2}^{\top} J e \\
L_{\omega} \Delta_{4}+\Lambda \Delta_{4} & =N_{1}^{\top} J e .
\end{aligned}
$$

As $\left[D \tau^{\top} J e\right]_{\mathbb{T}^{r}}=0$, the solution of equation (38) is unique, up to an arbitrary average $\left[\Delta_{2}\right]_{\mathbb{T}^{r}}$, provided that the non-resonance condition (2) holds. Then, using the non-degeneracy condition $\operatorname{det}\left[A_{1}\right]_{\mathbb{T}^{r}} \neq 0$, we choose

$$
\left[\Delta_{2}\right]_{\mathbb{T}^{r}}=\left[A_{1}\right]_{\mathbb{T}^{r}}^{-1}\left(\left[-V^{\top} J e\right]_{\mathbb{T}^{r}}-\left[\tilde{A}_{1} \tilde{\Delta}_{2}\right]_{\mathbb{T}^{r}}\right)
$$

in such a way that $\left[A_{1} \Delta_{2}+V^{\top} J e\right]_{\mathbb{T}^{r}}=0$ so we have a unique solution for $\Delta_{1}$ up to the freedom of fixing $\left[\Delta_{1}\right]_{\mathbb{T}^{r}}$. Actually, it is easy to check (39) and (40) have unique solution for $\Delta_{3}$ and $\Delta_{4}$ provided that the non-resonance condition (9) is fulfilled. Moreover, since $e$ is a real function, we conclude that $\Delta_{3}^{*}=\mathrm{i} \Delta_{4}$ and this allows us to guarantee that the expression (36) is also real.

Remark 4.5. We will see that the compatibility condition $\left[D \tau^{\top} J e\right]_{\mathbb{T}^{r}}=0$ is automatically fulfilled if $\tau$ parametrices and approximately invariant torus, e being the error of invariance - see computations in (93).

Proposition 4.6. Under the setting of Proposition 4.1, given a function $\hat{R}: \mathbb{T}^{r} \mapsto M_{2 n \times(n-r)}(\mathbb{C})$, we obtain a solution for the equation

$$
\mathcal{S}\left(\Delta_{N}, \Delta_{\Lambda}\right)=\mathcal{R}\left(\Delta_{N}\right)+N \Delta_{\Lambda}+\Delta_{N} \Lambda=-\hat{R}
$$

which is unique for $\Delta_{\Lambda}$ and for $\Delta_{N}$ up to terms in $\operatorname{ker}(\mathcal{S})=\left\{N D: D=\operatorname{diag}(d), d \in \mathbb{C}^{n-r}\right\}$. Proof. As before, we write the solution $\Delta_{N}$ of this equation in terms of the symplectic basis as

$$
\Delta_{N}=D \tau P_{1}+V P_{2}+N_{1} P_{3}+N_{2} P_{4}
$$

Then, we compute the action of $\mathcal{S}$ on the pair $\left(\Delta_{N}, \Delta_{\Lambda}\right)$, thus obtaining

$$
\begin{aligned}
\mathcal{S}\left(\Delta_{N}, \Delta_{\Lambda}\right)= & \mathcal{R}\left(\Delta_{N}\right)+N_{1} \Delta_{\Lambda}+\Delta_{N} \Lambda \\
= & \mathcal{R}(D \tau) P_{1}+\mathcal{R}(V) P_{2}+\mathcal{R}\left(N_{1}\right) P_{3}+\mathcal{R}\left(N_{2}\right) P_{4} \\
& +D \tau L_{\omega} P_{1}+V L_{\omega} P_{2}+N_{1} L_{\omega} P_{3}+N_{2} L_{\omega} P_{4}+N_{1} \Delta_{\Lambda} \\
& +D \tau P_{1} \Lambda+V P_{2} \Lambda+N_{1} P_{3} \Lambda+N_{2} P_{4} \Lambda
\end{aligned}
$$




$$
\begin{aligned}
= & D \tau\left(L_{\omega} P_{1}+P_{1} \Lambda+A_{1} P_{2}\right)+V\left(L_{\omega} P_{2}+P_{2} \Lambda\right) \\
& +N_{1}\left(L_{\omega} P_{3}+P_{3} \Lambda-\Lambda P_{3}+\Delta_{\Lambda}\right)+N_{2}\left(L_{\omega} P_{4}+P_{4} \Lambda+\Lambda P_{4}\right)=\hat{R} .
\end{aligned}
$$

If we multiply this expression by $V^{\top} J, D \tau^{\top} J, N_{2}^{\top} J$ and $N_{1}^{\top} J$, we end up with the following four cohomological equations:

$$
\begin{aligned}
L_{\omega} P_{1}+P_{1} \Lambda+A_{1} P_{2} & =-V^{\top} J \hat{R}, \\
L_{\omega} P_{2}+P_{2} \Lambda & =D \tau^{\top} J \hat{R}, \\
L_{\omega} P_{3}+P_{3} \Lambda-\Lambda P_{3} & =-N_{2}^{\top} J \hat{R}-\Delta_{\Lambda}, \\
L_{\omega} P_{4}+P_{4} \Lambda+\Lambda P_{4} & =N_{1}^{\top} J \hat{R} .
\end{aligned}
$$

Let us observe that, under the assumed non-resonance conditions (7) and (8), the only unavoidable resonances are those in the diagonal of the average of equation (44), so we require that the diagonal of the average of the right-hand side of this equation vanishes. This is attained by fixing the correction of the normal eigenvalues $\Delta_{\Lambda}=-\operatorname{diag}\left[N_{2}^{\top} J \hat{R}\right]_{\mathbb{T}^{r}}$. Therefore, we obtain a unique solution $P_{1}, P_{2}, P_{3}, P_{4}$ and $\Delta_{\Lambda}$-modulo terms in diag $\left[P_{3}\right]_{\mathbb{T}^{r}}$ - of this system of equations.

Remark 4.7. We will see that if $\hat{R}$ corresponds to the error in reducibility as defined in equation (15) then the geometry imposes that the correction $\Delta_{\Lambda}$ is a pure imaginary diagonal matrix, thus preserving the elliptic normal behavior —-see computations in (96).

\section{One step of the Newton method}

In this section we perform one step of the Newton method to correct an approximately invariant and elliptic torus. To this end, we follow the scheme presented in Section 4.2 for the case of a true elliptic invariant torus. The main difficulty is that we have to handle with "noise" introduced by the approximately invariant an reducible character.

Proposition 5.1. Let us consider a Hamiltonian $h: U \subset \mathbb{R}^{2 n} \rightarrow \mathbb{R}$, where $U$ is an open set, and a vector of basic frequencies $\omega \in \mathbb{R}^{r}$. Let us assume that the following hypotheses hold:

$\mathrm{H}_{1}$ The Hamiltonian $h$ is real analytic and can be holomorphically extended to some complex neighborhood $\mathcal{U}$ of $U$. Moreover, we assume that $\|h\|_{\mathcal{C}^{3}, \mathcal{U}} \leq \sigma_{0}$.

$\mathrm{H}_{2}$ There exists an approximate invariant and elliptic torus in the sense of Definitions 2.5 and 2.9, i.e., we have an embedding $\tau$, a matrix function $N$ and approximated normal eigenvalues $\Lambda=\operatorname{diag}(\mathrm{i} \lambda)$, with $\lambda \in \mathbb{R}^{n-r}$, satisfying

$$
\begin{aligned}
L_{\omega} \tau(\theta) & =J \operatorname{grad} h(\tau(\theta))+e(\theta), \\
L_{\omega} N(\theta) & =J \operatorname{hess} h(\tau(\theta)) N(\theta)-N(\theta) \Lambda+R(\theta),
\end{aligned}
$$


for certain error functions $e$ and $R$, where the functions $\tau$ and $N$ are analytic and can be holomorphically extended to $\Delta(\rho)$ for certain $0<\rho<1$, satisfying $\tau(\Delta(\rho)) \subset \mathcal{U}$. Assume also that we have constants $\sigma_{1}, \sigma_{2}$ such that

$$
\|D \tau\|_{\rho},\|N\|_{\rho},\left\|G_{D \tau}^{-1}\right\|_{\rho},\left\|G_{N, N^{*}}^{-1}\right\|_{\rho}<\sigma_{1}, \quad \operatorname{dist}(\tau(\Delta(\rho)), \partial \mathcal{U})>\sigma_{2}>0 .
$$

$\mathrm{H}_{3}$ We have diag $\left[\Omega_{N, N^{*}}\right]_{\mathbb{T}^{r}}=\mathrm{iId}_{n-r}$.

$\mathrm{H}_{4}$ The real symmetric matrix $A_{1}$ given by

$$
A_{1}(\theta)=G_{D \tau}^{-1}(\theta) D \tau(\theta)^{\top}\left(T_{1}(\theta)+T_{2}(\theta)+T_{2}(\theta)^{\top}\right) D \tau(\theta) G_{D \tau}^{-1}(\theta),
$$

where

$$
\begin{aligned}
& T_{1}(\theta)=J^{\top} \text { hess } h(\tau(\theta)) J-\text { hess } h(\tau(\theta)), \\
& T_{2}(\theta)=T_{1}(\theta) J\left[D \tau(\theta) G_{D \tau}(\theta)^{-1} D \tau(\theta)^{\top}-\mathrm{Id}\right] \operatorname{Re}\left(\mathrm{i} N(\theta) N^{*}(\theta)^{\top}\right),
\end{aligned}
$$

satisfies the non-degeneracy (twist) condition $\left|\left[A_{1}\right]_{\mathbb{T}^{r}}^{-1}\right|<\sigma_{1}$.

$\mathrm{H}_{5}$ There exist constants $\sigma_{3}, \sigma_{4}$ such that the approximated normal frequencies satisfy

$$
0<\frac{\sigma_{3}}{2}<\left|\lambda_{i}\right|<\frac{\sigma_{4}}{2}, \quad 0<\sigma_{3}<\left|\lambda_{i} \pm \lambda_{j}\right|,
$$

for $i, j=1, \ldots, n-r$, with $i \neq j$.

$\mathrm{H}_{6}$ The basic frequencies $\omega \in \mathbb{R}^{r}$ and the normal frequencies $\lambda \in \mathbb{R}^{n-r}$ satisfy Diophantine conditions (4) and (9) of ( $\gamma, \nu)$-type, for certain $0<\gamma<1$ and $\nu>r-1$.

Then, there exist a constant $\bar{\alpha}>1$ depending on $\nu, r, n,|\omega|, \sigma_{0}, \sigma_{1}, \sigma_{2}, \sigma_{3}$ and $\sigma_{4}$ such that if the following bounds are satisfied

$$
\begin{aligned}
& \frac{\bar{\alpha}}{\gamma^{4} \delta^{4 \nu-1}}\left(\frac{\|e\|_{\rho}}{\delta}+\|R\|_{\rho}\right)<\min \left\{1, \sigma_{1}-\sigma^{*}\right\} \\
& \operatorname{dist}(\tau(\Delta(\rho)), \partial \mathcal{U})-\frac{\bar{\alpha}}{\gamma^{2} \delta^{2 \nu}}\|e\|_{\rho}>\sigma_{2} \\
& \min _{i \neq j}\left|\lambda_{i} \pm \lambda_{j}\right|-\frac{\bar{\alpha}}{\gamma^{2} \delta^{2 \nu-1}}\left(\frac{\|e\|_{\rho}}{\delta}+\|R\|_{\rho}\right)>\sigma_{3} \\
& \min _{i}\left|\lambda_{i}\right|-\frac{\bar{\alpha}}{\gamma^{2} \delta^{2 \nu-1}}\left(\frac{\|e\|_{\rho}}{\delta}+\|R\|_{\rho}\right)>\frac{\sigma_{3}}{2} \\
& \max _{i}\left|\lambda_{i}\right|+\frac{\bar{\alpha}}{\gamma^{2} \delta^{2 \nu-1}}\left(\frac{\|e\|_{\rho}}{\delta}+\|R\|_{\rho}\right)<\frac{\sigma_{4}}{2}
\end{aligned}
$$


where

$$
\sigma^{*}=\max \left\{\|D \tau\|_{\rho},\|N\|_{\rho},\left\|G_{D \tau}^{-1}\right\|_{\rho},\left\|G_{N, N^{*}}^{-1}\right\|_{\rho},\left|\left[A_{1}\right]_{\mathbb{T}^{r}}^{-1}\right|\right\},
$$

for some $0<\delta<\rho / 4$, then we have an approximate invariant and elliptic torus $\overline{\mathscr{T}}$ for $X_{h}$ of the same basic frequencies $\omega$, i.e., we have an embedding $\bar{\tau}=\tau+\Delta_{\tau}$, with $\bar{\tau}\left(\mathbb{T}^{r}\right)=\overline{\mathscr{T}}$, a matrix function $N=N+\Delta_{N}$, which are analytic in $\Delta(\rho-2 \delta)$ and $\Delta(\rho-4 \delta)$, respectively, and approximated normal eigenvalues $\bar{\Lambda}=\operatorname{diag}(\mathrm{i} \bar{\lambda})=\Lambda+\Delta_{\Lambda}$, with $\bar{\lambda} \in \mathbb{R}^{n-r}$, such that

$$
\begin{aligned}
L_{\omega} \bar{\tau}(\theta) & =J \operatorname{grad} h(\bar{\tau}(\theta))+\bar{e}(\theta), \\
L_{\omega} \bar{N}(\theta) & =J \operatorname{hess} h(\bar{\tau}(\theta)) \bar{N}(\theta)-\bar{N}(\theta) \bar{\Lambda}+\bar{R}(\theta) .
\end{aligned}
$$

In addition, the following estimates hold

$$
\begin{aligned}
\left\|\Delta_{\tau}\right\|_{\rho-2 \delta} & \leq \frac{\bar{\alpha}}{\gamma^{2} \delta^{2 \nu}}\|e\|_{\rho} \\
\|\bar{e}\|_{\rho-3 \delta} & \leq \frac{\bar{\alpha}}{\gamma^{4} \delta^{4 \nu-1}}\left(\frac{\|e\|_{\rho}}{\delta}+\|R\|_{\rho}\right)\|e\|_{\rho}, \\
\left|\Delta_{\Lambda}\right| & \leq \frac{\bar{\alpha}}{\gamma^{2} \delta^{2 \nu-1}}\left(\frac{\|e\|_{\rho}}{\delta}+\|R\|_{\rho}\right) \\
\left\|\Delta_{N}\right\|_{\rho-4 \delta} & \leq \frac{\bar{\alpha}}{\gamma^{4} \delta^{4 \nu-1}}\left(\frac{\|e\|_{\rho}}{\delta}+\|R\|_{\rho}\right) \\
\|\bar{R}\|_{\rho-4 \delta} & \leq \frac{\bar{\alpha}}{\gamma^{8} \delta^{8 \nu-2}}\left(\frac{\|e\|_{\rho}}{\delta}+\|R\|_{\rho}\right)^{2} \\
\left\|G_{D \bar{\tau}}^{-1}-G_{D \tau}^{-1}\right\|_{\rho-3 \delta} & \leq \frac{\bar{\alpha}}{\gamma^{2} \delta^{2 \nu+1}}\|e\|_{\rho} \\
\left\|G_{\bar{N}, \bar{N}^{*}}^{-1}-G_{N, N^{*}}^{-1}\right\|_{\rho-4 \delta} & \leq \frac{\bar{\alpha}}{\gamma^{4} \delta^{4 \nu-1}}\left(\frac{\|e\|_{\rho}}{\delta}+\|R\|_{\rho}\right) \\
\left|\left[\bar{A}_{1}\right]_{\mathbb{T}^{r}}^{-1}-\left[A_{1}\right]_{\mathbb{T}^{r}}^{-1}\right| & \leq \frac{\bar{\alpha}}{\gamma^{4} \delta^{4 \nu-1}}\left(\frac{\|e\|_{\rho}}{\delta}+\|R\|_{\rho}\right) .
\end{aligned}
$$

Furthermore, the new objects satisfy the following conditions

$$
\operatorname{dist}(\bar{\tau}(\Delta(\rho-2 \delta)), \partial \mathcal{U})>\sigma_{2}, \quad \frac{\sigma_{3}}{2}<\left|\bar{\lambda}_{j}\right|<\frac{\sigma_{4}}{2}, \quad \sigma_{3}<\left|\bar{\lambda}_{i} \pm \bar{\lambda}_{j}\right|,
$$

for $i, j=1, \ldots, n-r$, with $i \neq j$, and

$$
\max \left\{\|D \bar{\tau}\|_{\rho-3 \delta},\|\bar{N}\|_{\rho-4 \delta},\left\|G_{D \bar{\tau}}^{-1}\right\|_{\rho-3 \delta},\left\|G_{\bar{N}, \bar{N}^{*}}^{-1}\right\|_{\rho-4 \delta},\left|\left[\bar{A}_{1}\right]_{\mathbb{T}^{r}}^{-1}\right|\right\}<\sigma_{1},
$$

where $\bar{A}_{1}$ corresponds to formulas (48), (49) and (50) for $\bar{\tau}$ and $\bar{N}$. Moreover, the columns of $\bar{N}$ are normalized in such a way that $\operatorname{diag}\left[\Omega_{\bar{N}, \bar{N}^{*}}\right]_{\mathbb{T}^{r}}=\mathrm{iId}_{n-r}$. 
To prove this result, we first construct an approximately symplectic basis along the torus following the ideas of Section 4.2. This is done in Proposition 5.3. The geometric properties of this basis will allow us to approximately invert the operators $\mathcal{R}$ and $\mathcal{S}$-given by (17) and (18), respectively - as it is required to obtain the iterative result of Proposition 5.1. Basically, it turns out that the solutions of the cohomological equations derived in Section 4.2 are enough to get the desired result. Before that, we state the following standard result that allows us to control the small divisors.

Lemma 5.2 (Rüssmann estimates). Let $g: \mathbb{T}^{r} \rightarrow \mathbb{C}$ be an analytic function on $\Delta(\rho)$ and bounded in the closure. Given $\omega \in \mathbb{R}^{r} \backslash\{0\}$ and $d \in \mathbb{R} \backslash\{0\}$ we consider the sets of complex numbers $\left\{d_{k}^{0}\right\}_{k \in \mathbb{Z}^{r} \backslash\{0\}},\left\{d_{k}^{1}\right\}_{k \in \mathbb{Z}^{r}}$ given by $d_{k}^{0}=\langle k, \omega\rangle, d_{k}^{1}=\langle k, \omega\rangle+d$, satisfying

$$
\left|d_{k}^{0}\right|,\left|d_{k}^{1}\right| \geq \gamma /|k|_{1}^{\nu}, \quad \forall k \in \mathbb{Z}^{r} \backslash\{0\}
$$

for certain $\gamma>0$ and $\nu>r-1$. Then, the functions $f^{0}$ and $f^{1}$ whose Fourier coefficients are given by

$$
\begin{aligned}
& \hat{f}_{k}^{0}=\hat{g}_{k} / d_{k}^{0}, \quad k \in \mathbb{Z}^{r} \backslash\{0\}, \quad \hat{f}_{0}^{0}=0, \\
& \hat{f}_{k}^{1}=\hat{g}_{k} / d_{k}^{1}, \quad k \in \mathbb{Z}^{r},
\end{aligned}
$$

satisfy

$$
\left\|f^{0}\right\|_{\rho-\delta} \leq \frac{\alpha_{0}}{\gamma \delta^{\nu}}\|g\|_{\rho}, \quad\left\|f^{1}\right\|_{\rho-\delta} \leq\left(\frac{1}{|d|}+\frac{\alpha_{0}}{\gamma \delta^{\nu}}\right)\|g\|_{\rho}
$$

for any $\delta \in(0, \min \{1, \rho\})$, where $\alpha_{0} \geq 1$ is a constant depending on $r$ and $\nu$.

Proof. We can control the functions $\tilde{f}^{i}(\theta)=f^{i}(\theta)-\left[f^{i}\right]_{\mathbb{T}^{r}}$ as

$$
\left\|\tilde{f}^{i}\right\|_{\rho-\delta} \leq \sum_{k \in \mathbb{Z}^{r} \backslash\{0\}} \frac{\left|\hat{g}_{k}\right|}{\left|d_{k}^{i}\right|} \mathrm{e}^{|k|_{1}(\rho-\delta)} \leq\left(\sum_{k \in \mathbb{Z}^{r} \backslash\{0\}}\left|\hat{g}_{k}\right|^{2} \mathrm{e}^{2|k|_{1} \rho}\right)^{1 / 2}\left(\sum_{k \in \mathbb{Z}^{r} \backslash\{0\}} \frac{1}{\left|d_{k}^{i}\right|^{2}} \mathrm{e}^{-2|k|_{1} \delta}\right)^{1 / 2},
$$

for $i=0,1$, where we used Cauchy-Schwarz inequality. On the one hand, it is not difficult to see — using Bessel's inequality, see details in [57]— that the first term can be bounded by

$$
\sum_{k \in \mathbb{Z}^{r} \backslash\{0\}}\left|\hat{g}_{k}\right|^{2} \mathrm{e}^{2|k|_{1} \rho} \leq 2^{r}\|\tilde{g}\|_{\rho}^{2},
$$

and on the other hand, the second term is controlled by estimating the sum

$$
\sum_{k \in \mathbb{Z}^{r} \backslash\{0\}} \frac{1}{\left|d_{k}^{i}\right|^{2}} \mathrm{e}^{-2|k|_{1} \delta}=\sum_{l=1}^{\infty}\left(\sum_{\substack{k \in \mathbb{Z}^{r} \backslash\{0\} \\|k|_{1} \leq l}} \frac{1}{\left|d_{k}^{i}\right|^{2}}\right)\left(\mathrm{e}^{-2 l \delta}-\mathrm{e}^{-2(l+1) \delta}\right) .
$$


Now, we study in detail the case of $d_{k}^{1}$ (the case of $d_{k}^{0}$ is analogous). First, we observe that the divisors $d_{k}^{1}=\langle k, \omega\rangle+d$ satisfy $d_{k_{1}} \neq d_{k_{2}}$ if $k_{1} \neq k_{2}$. Then, given $l \in \mathbb{N}$, we define

$$
\mathcal{D}_{l}=\left\{k \in \mathbb{Z}^{r} \backslash\{0\}:|k|_{1} \leq l \text { and } d_{k}^{1}>0\right\}
$$

and we sort the divisors according to $0<d_{k_{1}}<\ldots<d_{k_{\# \mathcal{D}_{l}}}$ with $k_{j} \in \mathcal{D}_{l}$, for $j=1, \ldots, \# \mathcal{D}_{l}$. Then, we observe that (since $\left|k_{j}-k_{j-1}\right| \leq 2 l$ )

$$
d_{k_{j}}^{1}-d_{k_{j-1}}^{1}=\left|\left\langle k_{j}-k_{j-1}, \omega\right\rangle\right| \geq d_{2 l, \min }^{0}
$$

where the have introduced the notation

$$
d_{l, \min }^{i}=\min _{\substack{k \in \mathbb{Z}^{r} \backslash\{0\} \\|k|_{1} \leq l}}\left|d_{k}^{i}\right|
$$

From expression (67) we obtain recursively

$$
d_{k_{j}}^{1}=d_{k_{j-1}}^{1}+d_{k_{j}}^{1}-d_{k_{j-1}}^{1} \geq d_{k_{j-1}}^{1}+d_{2 l, \min }^{0} \geq d_{l, \text { min }}^{1}+(j-1) d_{2 l, \min }^{0} .
$$

Then, using that $d_{2 l, \text { min }}^{0} \geq \gamma /(2 l)^{\nu}$ and $d_{l, \text { min }}^{1} \geq \gamma / l^{\nu}$, we have

$$
\sum_{j=1}^{\# \mathcal{D}_{l}} \frac{1}{\left(d_{k_{j}}^{1}\right)^{2}} \leq \sum_{j=1}^{\# \mathcal{D}_{l}} \frac{1}{\left(d_{l, \text { min }}^{1}+(j-1) d_{2 l, \text { min }}^{0}\right)^{2}} \leq \sum_{j=1}^{\infty} \frac{l^{2 \nu}}{\gamma^{2}\left(1+(j-1) 2^{-\nu}\right)^{2}} \leq \frac{\alpha(\nu)}{\gamma^{2}} l^{2 \nu},
$$

and using a similar argument for $d_{k_{j}}^{1}<0$, we obtain

$$
\sum_{\substack{k \in \mathbb{Z}^{r} \backslash\{0\} \\|k|_{1} \leq l}} \frac{1}{\left|d_{k}^{1}\right|^{2}} \leq \frac{2 \alpha(\nu)}{\gamma^{2}} l^{2 \nu}
$$

so we can control the sum (66) as follows

$$
\sum_{k \in \mathbb{Z}^{r} \backslash\{0\}} \frac{1}{\left|d_{k}^{i}\right|^{2}} \mathrm{e}^{-2|k|_{1} \delta} \leq \sum_{l=1}^{\infty} \frac{2 \delta \alpha(\nu)}{\gamma^{2}} \int_{l}^{l+1} x^{2 \nu} \mathrm{e}^{-2 \delta x} d x \leq \frac{\alpha(\nu)}{\gamma^{2}(2 \delta)^{2 \nu}} \Gamma(2 \nu+1) .
$$

Combining the obtained expressions — and using that $\left|\left[f^{1}\right]_{\mathbb{T}^{r}}\right|=\left|\hat{g}_{0}\right| /|d|$ - we end up with the stated estimates.

Proposition 5.3. Under the same notations and assumptions of Proposition 5.1, we define the matrix functions

$$
N_{1}(\theta)=N(\theta), \quad N_{2}(\theta)=\mathrm{i} N^{*}(\theta)
$$


and the real analytic matrix $V(\theta)$ given by (23)-(26). Then, for any $0<\delta<\rho / 2$ the following estimates hold:

$$
\begin{aligned}
\left\|\Omega_{D \tau}\right\|_{\rho-2 \delta} & \leq \frac{\hat{\alpha}}{\gamma \delta^{\nu+1}}\|e\|_{\rho}, \\
\left\|\Omega_{N_{i}}\right\|_{\rho-\delta} & \leq \frac{\hat{\alpha}}{\gamma \delta^{\nu}}\|R\|_{\rho}, \\
\left\|\Omega_{D \tau, N_{i}}\right\|_{\rho-2 \delta} & \leq \frac{\hat{\alpha}}{\gamma \delta^{\nu}}\left(\frac{\|e\|_{\rho}}{\delta}+\|R\|_{\rho}\right), \\
\left\|\Omega_{N_{2}, N_{1}}-\operatorname{Id}_{n-r}\right\|_{\rho-\delta} & \leq \frac{\hat{\alpha}}{\gamma \delta^{\nu}}\|R\|_{\rho}, \\
\left\|\Omega_{V, D \tau}-\operatorname{Id}_{r}\right\|_{\rho-2 \delta} & \leq \frac{\hat{\alpha}}{\gamma \delta^{\nu}}\left(\frac{\|e\|_{\rho}}{\delta}+\|R\|_{\rho}\right), \\
\left\|\Omega_{V, N_{i}}\right\|_{\rho-2 \delta} & \leq \frac{\hat{\alpha}}{\gamma \delta^{\nu}}\left(\frac{\|e\|_{\rho}}{\delta}+\|R\|_{\rho}\right), \\
\left\|\Omega_{V}\right\|_{\rho-2 \delta} & \leq \frac{\hat{\alpha}}{\gamma \delta^{\nu}}\left(\frac{\|e\|_{\rho}}{\delta}+\|R\|_{\rho}\right),
\end{aligned}
$$

for $i=1,2$, where $\hat{\alpha}>1$ is a constant depending on $\nu, r, n,|\omega|, \sigma_{0}, \sigma_{1}, \sigma_{3}$ and $\sigma_{4}$. Furthermore, if the errors $\|e\|_{\rho}$ and $\|R\|_{\rho}$ satisfy

$$
\frac{\hat{\alpha}}{\gamma \delta^{\nu}}\left(\frac{\|e\|_{\rho}}{\delta}+\|R\|_{\rho}\right) \leq \frac{1}{2}
$$

then the columns of $D \tau(\theta), V(\theta), N_{1}(\theta), N_{2}(\theta)$ form an approximately symplectic basis for every $\theta \in \mathbb{T}^{r}$. In addition, it turns out that the action of the operator $\mathcal{R}$ given in (17) on $V$ is expressed in terms of this basis as

$$
\mathcal{R}(V(\theta))=D \tau(\theta)\left(A_{1}(\theta)+A_{1}^{+}(\theta)\right)+V(\theta) A_{2}^{+}(\theta)+N_{1}(\theta) A_{3}^{+}(\theta)+N_{2}(\theta) A_{4}^{+}(\theta),
$$

where $A_{1}$ is the matrix (48) and $A_{1}^{+}, A_{2}^{+}, A_{3}^{+}$and $A_{4}^{+}$satisfy the estimate

$$
\left\|A_{i}^{+}\right\|_{\rho-2 \delta} \leq \frac{\hat{\alpha}}{\gamma \delta^{\nu+1}}\left(\frac{\|e\|_{\rho}}{\delta}+\|R\|_{\rho}\right),
$$

for $i=1, \ldots, 4$.

Proof. For the sake of simplicity, we redefine (enlarge) the constant $\hat{\alpha}$ along the proof to meet the different conditions given in the statement. For example, we observe that there exist a constant $\hat{\alpha}>0$, depending on $r, n,|\omega|, \sigma_{0}$ and $\sigma_{1}$, such that

$$
\left\|B_{i}\right\|_{\rho},\left\|T_{1}\right\|_{\rho},\left\|T_{2}\right\|_{\rho},\left\|A_{1}\right\|_{\rho},\|V\|_{\rho} \leq \hat{\alpha}, \quad\left\|L_{\omega} B_{i}\right\|_{\rho-\delta} \leq \frac{\hat{\alpha}}{\delta},
$$


for $i=1,2,3$-we recall that $T_{1}$ and $T_{2}$ are given in (49) and (50), respectively. Now we take derivatives at both sides of the approximated invariance equation in (46) and we read the reducibility equations in (47) for $N_{1}$ and $N_{2}$

$$
\begin{aligned}
L_{\omega} D \tau & =J \operatorname{hess} h(\tau) D \tau+D e, \\
L_{\omega} N_{1} & =J \operatorname{hess} h(\tau) N_{1}-N_{1} \Lambda+R, \\
L_{\omega} N_{2} & =J \operatorname{hess} h(\tau) N_{2}+N_{2} \Lambda+\mathrm{i} R^{*} .
\end{aligned}
$$

Using the previous expressions, we compute the derivate $L_{\omega}$ of the matrices $\Omega_{D \tau}, \Omega_{N_{1}}$, $\Omega_{D \tau, N_{1}}$ and $\Omega_{N_{2}, N_{1}}$ thus obtaining

$$
\begin{aligned}
L_{\omega}\left(\Omega_{D \tau}\right) & =\Omega_{D e, D \tau}+\Omega_{D \tau, D e} \\
L_{\omega}\left(\Omega_{N_{1}}\right) & =-\Lambda \Omega_{N_{1}}-\Omega_{N_{1}} \Lambda+\Omega_{R, N_{1}}+\Omega_{N_{1} R}, \\
L_{\omega}\left(\Omega_{D \tau, N_{1}}\right) & =-\Omega_{D \tau, N_{1}} \Lambda+\Omega_{D e, N_{1}}+\Omega_{D \tau, R}, \\
L_{\omega}\left(\Omega_{N_{2}, N_{1}}\right) & =\Lambda \Omega_{N_{2}, N_{1}}-\Omega_{N_{2}, N_{1}} \Lambda+\mathrm{i} \Omega_{R^{*}, N_{1}}+\Omega_{N_{2}, R} .
\end{aligned}
$$

First, we get estimate (68) for $\Omega_{D \tau}$ by applying Lemma 5.2 to the $(i, j)$-component of $\Omega_{D \tau}$ obtained from (80), i.e., taking $d_{k}^{0}=\langle\omega, k\rangle$ and $g=-\mathrm{i}\left(\Omega_{D e, D \tau}+\Omega_{D \tau, D e}\right)^{(i, j)}$ that (using Cauchy estimates) is analytic in $\Delta(\rho-\delta)$. Moreover, since $\left[\Omega_{D \tau}\right]_{\mathbb{T}^{r}}=0$ (see Remark 2.2), we obtain

$$
\left\|\Omega_{D \tau}\right\|_{\rho-2 \delta} \leq \frac{\alpha_{0}}{\gamma \delta^{\nu}}\|g\|_{\rho-\delta} \leq \frac{\hat{\alpha}}{\gamma \delta^{\nu+1}}\|e\|_{\rho}
$$

Then, we proceed in a similar way to get (69) for $N_{1}$, by applying Lemma 5.2 to the $(i, j)$ component of $\Omega_{N_{1}}$ obtained from (81), i.e., taking $d_{k}^{1}=\langle\omega, k\rangle+\lambda_{i}+\lambda_{j}$ and $g=-\mathrm{i}\left(\Omega_{R, N_{1}}+\right.$ $\left.\Omega_{N_{1} R}\right)^{(i, j)}$, analytic in $\Delta(\rho)$. To bound the average of $\Omega_{N_{1}}$, we use hypothesis $\mathrm{H}_{5}$ of Proposition 5.1.

$$
\left\|\Omega_{N_{1}}\right\|_{\rho-\delta} \leq\left(\frac{1}{\min _{i, j}\left|\lambda_{i}+\lambda_{j}\right|}+\frac{\alpha_{0}}{\gamma \delta^{\nu}}\right)\|g\|_{\rho} \leq\left(\frac{1}{\sigma_{3}}+\frac{\alpha_{0}}{\gamma \delta^{\nu}}\right) \leq \frac{\hat{\alpha}}{\gamma \delta^{\nu}}\|R\|_{\rho},
$$

Analogous computations from Equations (82) and (83) allow us to obtain estimate (70) for $N_{1}$ and (71). Of course, to obtain (71) we resort to the hypothesis diag $\left[\Omega_{N, N^{*}}\right]_{\mathbb{T}^{r}}=\mathrm{iId}_{n-r}$ in $\mathrm{H}_{3}$ of Proposition 5.1. The corresponding estimates (69) and (70) for $N_{2}$ are straightforward using that $\Omega_{N_{2}}=-\Omega_{N_{1}}^{*}$ and $\Omega_{D \tau, N_{2}}=\mathrm{i} \Omega_{D \tau, N_{1}}^{*}$.

Next we show that the columns of $D \tau, J D \tau G_{D \tau}^{-1}, \operatorname{Re}\left(N_{1}\right)$ and $\operatorname{Im}\left(N_{1}\right)$ form a $\mathbb{R}$-basis of $\mathbb{R}^{2 n}$. As in the proof of Proposition 4.1, we consider a linear combination

$$
D \tau a+J D \tau G_{D \tau}^{-1} b+N_{1} c+N_{2} d=0
$$


for functions $a, b: \mathbb{T}^{r} \rightarrow \mathbb{C}^{r}$ and $c, d: \mathbb{T}^{r} \rightarrow \mathbb{C}^{n-r}$. We project this equation multiplying by $D \tau^{\top}, D \tau^{\top} J, N_{2}^{\top} J$ and $N_{1}^{\top} J$, thus obtaining

$$
(M_{1}+\underbrace{\left(\begin{array}{cccc}
0 & \Omega_{D \tau} G_{D \tau}^{-1} & 0 & 0 \\
\Omega_{D \tau} & 0 & \Omega_{D \tau, N_{1}} & \Omega_{D \tau, N_{2}} \\
\Omega_{N_{2}, D \tau} & 0 & \Omega_{N_{2}, N_{1}}-\mathrm{Id}_{n-r} & \Omega_{N_{2}} \\
\Omega_{N_{1}, D \tau} & 0 & \Omega_{N_{1}} & \Omega_{N_{1}, N_{2}}+\mathrm{Id}_{n-r}
\end{array}\right)}_{M_{2}})\left(\begin{array}{l}
a \\
b \\
c \\
d
\end{array}\right)=\left(\begin{array}{l}
0 \\
0 \\
0 \\
0
\end{array}\right),
$$

where $M_{1}$ is the same matrix that appears in equation (27). Now, we have to invert the matrix $M_{1}+M_{2}=M_{1}\left(\mathrm{Id}+M_{1}^{-1} M_{2}\right)$, where

$$
M_{1}^{-1}=\left(\begin{array}{cccc}
G_{D \tau}^{-1} & M_{1,2} & -G_{D \tau}^{-1} G_{D \tau, N_{1}} & G_{D \tau}^{-1} G_{D \tau, N_{2}} \\
0 & -\mathrm{Id}_{r} & 0 & 0 \\
0 & -G_{N_{2}, D \tau} G_{D \tau}^{-1} & \operatorname{Id}_{n-r} & 0 \\
0 & G_{N_{1}, D \tau} G_{D \tau}^{-1} & 0 & -\operatorname{Id}_{n-r}
\end{array}\right),
$$

with $M_{1,2}=G_{D \tau}^{-1}\left(G_{D \tau, N_{1}} G_{N_{2}, D \tau}-G_{D \tau, N_{2}} G_{N_{1}, D \tau}\right) G_{D \tau}^{-1}$, so it is clear that $\left\|M_{1}^{-1}\right\|_{\rho} \leq \hat{\alpha}$. By means of Neumann series we obtain

$$
\left\|\left(\mathrm{Id}+M_{1}^{-1} M_{2}\right)^{-1}\right\|_{\rho-2 \delta} \leq \frac{1}{1-\left\|M_{1}^{-1} M_{2}\right\|_{\rho-2 \delta}},
$$

that it is well posed since (using bounds (68)-(71))

$$
\left\|M_{1}^{-1} M_{2}\right\|_{\rho-2 \delta} \leq \frac{\hat{\alpha}}{\gamma \delta^{\nu}}\left(\frac{\|e\|_{\rho}}{\delta}+\|R\|_{\rho}\right) \leq \frac{1}{2}
$$

and applying hypothesis (75). Then, it must be $a=b=0$ and $c=d=0$ along $\mathbb{T}^{r}$.

Now, we consider the basis defined by the columns of $D \tau, V, N_{1}$ and $N_{2}$, where $V$ is given by (23)-(26), and we characterize the fact that the new basis is approximately symplectic. It is straightforward to compute

$$
\begin{aligned}
\Omega_{D \tau, V} & =-\operatorname{Id}_{r}+\Omega_{D \tau, N_{1}} B_{1}+\Omega_{D \tau, N_{2}} B_{2}+\Omega_{D \tau} B_{3}, \\
\Omega_{N_{1}, V} & =-G_{N_{1}, D \tau} G_{D \tau}^{-1}+\Omega_{N_{1}} B_{1}+\Omega_{N_{1}, N_{2}} B_{2}+\Omega_{N_{1}, D \tau} B_{3} \\
& =\Omega_{N_{1}} B_{1}+\left(\Omega_{N_{1}, N_{2}}+\operatorname{Id}_{n-r}\right) B_{2}+\Omega_{N_{1}, D \tau} B_{3}, \\
\Omega_{V} & =B_{3}^{\top}\left(\Omega_{D \tau, V}+\operatorname{Id}_{r}\right)+B_{1}^{\top} \Omega_{N_{1}, V}+B_{2}^{\top} \Omega_{N_{2}, V}+G_{D \tau}^{-1} \Omega_{D \tau} G_{D \tau}^{-1},
\end{aligned}
$$

and $\Omega_{N_{2}, V}=\mathrm{i} \Omega_{N_{1}, V}^{*}$. Then, estimates (72)-(74) follow from (68)-(71) and (78).

Let us characterize the action of the linear operator $\mathcal{R}$ on the elements of this basis. By hypothesis, we immediately have that

$$
\mathcal{R}(D \tau)=D e, \quad \mathcal{R}\left(N_{1}\right)=-N_{1} \Lambda+R, \quad \mathcal{R}\left(N_{2}\right)=N_{2} \Lambda+\mathrm{i} R^{*},
$$


and we have to see that if we write

$$
\mathcal{R}(V)=D \tau\left(A_{1}+A_{1}^{+}\right)+V A_{2}^{+}+N_{1} A_{3}^{+}+N_{2} A_{4}^{+},
$$

where $A_{1}$ is the matrix (29), then the functions $A_{1}^{+}, A_{2}^{+}, A_{3}^{+}$and $A_{4}^{+}$are small -i.e., they satisfy (77). To this end, expanding $\mathcal{R}(V)$ in the previous expression as

$$
\begin{aligned}
\mathcal{R}(V)= & \mathcal{R}\left(J D \tau G_{D \tau}^{-1}\right)+N_{1}\left(L_{\omega} B_{1}-\Lambda B_{1}\right)+N_{2}\left(L_{\omega} B_{2}+\Lambda B_{2}\right)+D \tau L_{\omega} B_{3} \\
& +R B_{1}+\mathrm{i} R^{*} B_{2}+D e B_{3},
\end{aligned}
$$

and multiplying at both sides of this equation by $V^{\top} J, D \tau^{\top} J, N_{2}^{\top} J$ and $N_{1}^{\top} J$, we obtain the linear system

$$
\left(\mathrm{Id}+M_{3}\right)\left(\begin{array}{c}
A_{1}^{+} \\
A_{2}^{+} \\
A_{3}^{+} \\
A_{4}^{+}
\end{array}\right)=\left(\begin{array}{l}
C_{1} \\
C_{2} \\
C_{3} \\
C_{4}
\end{array}\right)
$$

where

$$
M_{3}=\left(\begin{array}{cccc}
\Omega_{V, D \tau}-\mathrm{Id}_{r} & \Omega_{V} & \Omega_{V, N_{1}} & \Omega_{V, N_{2}} \\
-\Omega_{D \tau} & \Omega_{V, D \tau}-\mathrm{Id}_{r} & -\Omega_{D \tau, N_{1}} & -\Omega_{D \tau, N_{2}} \\
\Omega_{N_{2}, D \tau} & \Omega_{N_{2}, V} & \Omega_{N_{2}, N_{1}}-\mathrm{Id}_{n-r} & \Omega_{N_{2}} \\
-\Omega_{N_{1}, D \tau} & -\Omega_{N_{1}, V} & -\Omega_{N_{1}} & \Omega_{N_{2}, N_{1}}-\mathrm{Id}_{n-r}
\end{array}\right)
$$

and the functions $C_{1}, C_{2}, C_{3}$ and $C_{4}$ have the following form

$$
\begin{aligned}
C_{1}= & \overbrace{V^{\top} J \mathcal{R}\left(J D \tau G_{D \tau}^{-1}\right)+L_{\omega} B_{3}-A_{1}}^{C_{1}^{+}}+\Omega_{V, N_{1}}\left(L_{\omega} B_{1}-\Lambda B_{1}\right)+\Omega_{V, N_{2}}\left(L_{\omega} B_{2}+\Lambda B_{2}\right) \\
& +V^{\top} J\left(R B_{1}+\mathrm{i} R^{*} B_{2}+D e B_{3}\right)+\left(\Omega_{V, D \tau}-\mathrm{Id}_{r}\right)\left(L_{\omega} B_{3}-A_{1}\right), \\
C_{2}= & \overbrace{-D \tau^{\top} J \mathcal{R}\left(J D \tau G_{D \tau}^{-1}\right)}^{C_{2}^{+}}-\Omega_{D \tau, N_{1}}\left(L_{\omega} B_{1}-\Lambda B_{1}\right)-\Omega_{D \tau, N_{2}}\left(L_{\omega} B_{2}+\Lambda B_{2}\right) \\
& -D \tau^{\top} J\left(R B_{1}+\mathrm{i} R^{*} B_{2}+D e B_{3}\right)+\Omega_{D \tau}\left(A_{1}-L_{\omega} B_{3}\right), \\
C_{3}= & \overbrace{N_{2}^{\top} J \mathcal{R}\left(J D \tau G_{D \tau}^{-1}\right)+L_{\omega} B_{1}-\Lambda B_{1}}^{C_{3}^{+}}+\Omega_{N_{2}}\left(L_{\omega} B_{2}+\Lambda B_{2}\right)+\Omega_{N_{2}, D \tau}\left(L_{\omega} B_{3}-A_{1}\right) \\
& +\left(\Omega_{N_{2}, N_{1}}-\operatorname{Id}_{n-r}\right)\left(L_{\omega} B_{1}-\Lambda B_{1}\right)+N_{2}^{\top} J\left(R B_{1}+\mathrm{i} R^{*} B_{2}+D e B_{3}\right), \\
C_{4}= & \overbrace{-N_{1}^{\top} J \mathcal{R}\left(J D \tau G_{D \tau}^{-1}\right)+L_{\omega} B_{2}+\Lambda B_{2}}^{C_{4}^{+}}-\Omega_{N_{1}}\left(L_{\omega} B_{1}-\Lambda B_{1}\right)+\Omega_{N_{1}, D \tau}\left(A_{1}-L_{\omega} B_{3}\right) \\
& -\left(\Omega_{N_{1}, N_{2}}+\operatorname{Id}_{n-r}\right)\left(L_{\omega} B_{2}+\Lambda B_{2}\right)-N_{1}^{\top} J\left(R B_{1}+\mathrm{i} R^{*} B_{2}+D e B_{3}\right),
\end{aligned}
$$


and we observe that $C_{4}=-\mathrm{i} C_{3}^{*}$ and $C_{4}^{+}=-\mathrm{i}\left(C_{3}^{+}\right)^{*}$. Apart from $C_{1}^{+}, C_{2}^{+}, C_{3}^{+}$and $C_{4}^{+}$, the size of the other terms that appear in the above expressions are easily controlled in terms of $\|e\|_{\rho}$ and $\|R\|_{\rho}$-using approximately symplectic properties in (68)-(74). We see next that $C_{j}^{+}$, for $j=1, \ldots, 4$, are also controlled in a similar way, since they are given by equation which are close to (32)-(35) for the invariant and reducible case. For example, using equation (24) for $B_{1}$ in the expressions of $C_{3}^{+}$we obtain

$$
C_{3}^{+}=\left(L_{\omega} N_{2}-J \text { hess } h N_{2}-N_{2} \Lambda\right)^{\top} D \tau G_{D \tau}^{-1}=\mathrm{i} G_{R^{*}, D \tau} G_{D \tau}^{-1},
$$

where we used equation (79). To control $C_{1}^{+}$and $C_{2}^{+}$we have to compute the action of $\mathcal{R}$ on the matrix $J D \tau G_{D \tau}^{-1}$

$$
\begin{aligned}
\mathcal{R}\left(J D \tau G_{D \tau}^{-1}\right)= & \mathcal{R}(J D \tau) G_{D \tau}^{-1}+J D \tau L_{\omega}\left(G_{D \tau}^{-1}\right)=\left(\mathrm{Id}+J D \tau G_{D \tau}^{-1} D \tau^{\top} J\right) T_{1} D \tau G_{D \tau}^{-1} \\
& +J D e G_{D \tau}^{-1}-J D \tau G_{D \tau}^{-1}\left[G_{D e, D \tau}+G_{D \tau, D e}\right] G_{D \tau}^{-1} .
\end{aligned}
$$

where $T_{1}$ is given by (49). Then, if we multiply this expression by $D \tau^{\top} J$ we get

$$
C_{2}^{+}=-G_{D e, D \tau} G_{D \tau}^{-1}
$$

and if we multiply by $V^{\top} J$ and use the definitions of $C_{2}^{+}, C_{3}^{+}$and $C_{4}^{+}$, we obtain

$$
\begin{aligned}
C_{1}^{+}= & \left(B_{3}^{\top} D \tau^{\top}+B_{2}^{\top} N_{2}^{\top}+B_{1}^{\top} N_{1}^{\top}+G_{D \tau}^{-1} D \tau^{\top} J^{\top}\right) J \mathcal{R}\left(J D \tau G_{D \tau}^{-1}\right)+L_{\omega} B_{3}-A_{1} \\
= & -B_{3}^{\top} C_{2}^{+}+B_{2}^{\top}\left(C_{3}^{+}-L_{\omega} B_{1}+\Lambda B_{1}\right)+B_{1}^{\top}\left(-C_{4}^{+}+L_{\omega} B_{2}+\Lambda B_{2}\right) \\
& +G_{D \tau}^{-1} D \tau^{\top} \mathcal{R}\left(J D \tau G_{D \tau}^{-1}\right)+L_{\omega} B_{3}-A_{1} \\
= & -B_{3}^{\top} C_{2}^{+}+B_{2}^{\top} C_{3}^{+}-B_{1}^{\top} C_{4}^{+}+G_{D \tau}^{-1} \Omega_{D \tau} G_{D \tau}^{-1} D \tau^{\top} J T_{1} D \tau G_{D \tau}^{-1} \\
& +G_{D \tau}^{-1} \Omega_{D \tau, D e} G_{D \tau}^{-1}-G_{D \tau}^{-1} \Omega_{D \tau} G_{D \tau}^{-1}\left(G_{D e, D \tau}+G_{D \tau, D e}\right) G_{D \tau}^{-1}+C_{1}^{++}
\end{aligned}
$$

where $C_{1}^{++}$is given as

$$
\begin{aligned}
C_{1}^{++} & =G_{D \tau}^{-1} D \tau^{\top} T_{1} D \tau G_{D \tau}^{-1}+B_{1}^{\top}\left(L_{\omega} B_{2}+\Lambda B_{2}\right)-B_{2}^{\top}\left(L_{\omega} B_{1}-\Lambda B_{1}\right)+L_{\omega} B_{3}-A_{1} \\
& =G_{D \tau}^{-1} D \tau^{\top} T_{1} D \tau G_{D \tau}^{-1}+\operatorname{Re}\left(L_{\omega} B_{2}^{\top} B_{1}-B_{2}^{\top} L_{\omega} B_{1}+2 B_{2}^{\top} \Lambda B_{1}\right)-A_{1},
\end{aligned}
$$

where we used that $B_{3}=\operatorname{Re}\left(G_{B_{2}, B_{1}}\right)$ and $\left(B_{1}^{\top}\left(L_{\omega} B_{2}+\Lambda B_{2}\right)\right)^{*}=-B_{2}^{\top}\left(L_{\omega} B_{1}-\Lambda B_{1}\right)$. By introducing the expression (48) for $A_{1}$, expanding $L_{\omega} B_{1}$ and $L_{\omega} B_{2}$ as in Lemma 4.3

$$
\begin{aligned}
L_{\omega} B_{2}= & -\Lambda B_{2}+N_{1}^{\top} J T_{1} D \tau G_{D \tau}^{-1}-N_{1}^{\top} D \tau G_{D \tau}^{-1} D \tau^{\top} J T_{1} D \tau G_{D \tau}^{-1} \\
& -G_{R, D \tau} G_{D \tau}^{-1}-G_{N_{1}, D e} G_{D \tau}^{-1}+G_{N_{1}, D \tau} G_{D \tau}^{-1}\left(G_{D e, D \tau}+G_{D \tau, D e}\right) G_{D \tau}^{-1} . \\
L_{\omega} B_{1}= & \Lambda B_{1}-N_{2}^{\top} J T_{1} D \tau G_{D \tau}^{-1}+N_{2}^{\top} D \tau G_{D \tau}^{-1} D \tau^{\top} J T_{1} D \tau G_{D \tau}^{-1} \\
& +\mathrm{i} G_{R^{*}, D \tau} G_{D \tau}^{-1}+G_{N_{2}, D e} G_{D \tau}^{-1}-G_{N_{2}, D \tau} G_{D \tau}^{-1}\left(G_{D e, D \tau}+G_{D \tau, D e}\right) G_{D \tau}^{-1},
\end{aligned}
$$


and using that $\operatorname{Re}\left(N_{1} N_{2}^{\top}\right)=-\operatorname{Re}\left(\left(N_{1} N_{2}^{\top}\right)^{\top}\right)$ and $\operatorname{Re}\left(G_{D \tau, R} G_{N_{2}, D \tau}\right)^{\top}=-\operatorname{Re}\left(\mathrm{i} G_{D \tau, N_{1}} G_{R^{*}, D \tau}\right)$, we obtain (after some cancellations)

$$
C_{1}^{++}=\operatorname{Re}\left(T_{3}+T_{3}^{\top}\right)
$$

where

$$
T_{3}=-G_{D \tau}^{-1}\left(G_{D \tau, R}+G_{D e, N_{1}}-\left(G_{D e, D \tau}+G_{D \tau, D e}\right) G_{D \tau}^{-1} G_{D \tau, N_{1}}\right) G_{N_{2}, D \tau} G_{D \tau}^{-1} .
$$

Now, we control the expressions (88), (87), (91) and (90) as

$$
\left\|C_{2}^{+}\right\|_{\rho-\delta} \leq \frac{\hat{\alpha}}{\delta}\|e\|_{\rho}, \quad\left\|C_{3}^{+}\right\|_{\rho-\delta},\left\|C_{4}^{+}\right\|_{\rho-\delta} \leq \hat{\alpha}\|R\|_{\rho}, \quad\left\|T_{3}\right\|_{\rho-\delta},\left\|C_{1}^{++}\right\|_{\rho-\delta} \leq \hat{\alpha}\left(\frac{\|e\|_{\rho}}{\delta}+\|R\|_{\rho}\right)
$$

and we use these bounds to control the expression (89) as follows

$$
\left\|C_{1}^{+}\right\|_{\rho-2 \delta} \leq \hat{\alpha}\left(\frac{\|e\|_{\rho}}{\gamma \delta^{\nu+1}}+\frac{\|e\|_{\rho}^{2}}{\gamma \delta^{\nu+2}}+\|R\|_{\rho}\right)
$$

and we use hypothesis (75) to get rid of the quadratic terms, thus obtaining

$$
\left\|C_{1}^{+}\right\|_{\rho-2 \delta} \leq \frac{\hat{\alpha}}{\gamma \delta^{\nu}}\left(\frac{\|e\|_{\rho}}{\delta}+\|R\|_{\rho}\right)
$$

Therefore, we have

$$
\left\|C_{i}\right\|_{\rho-2 \delta} \leq \frac{\hat{\alpha}}{\gamma \delta^{\nu+1}}\left(\frac{\|e\|_{\rho}}{\delta}+\|R\|_{\rho}\right)
$$

for $i=1, \ldots, 4$. Finally, we obtain estimates for the inverse of the matrix $\operatorname{Id}+M_{3}$ that appears in system (85), given by

$$
\left\|\left(\mathrm{Id}+M_{3}\right)^{-1}\right\|_{\rho-2 \delta} \leq \frac{1}{1-\left\|M_{3}\right\|_{\rho-2 \delta}},
$$

that, by using hypothesis (75) again, is well-posed since

$$
\left\|M_{3}\right\|_{\rho-2 \delta} \leq \frac{\hat{\alpha}}{\gamma \delta^{\nu}}\left(\frac{\|e\|_{\rho}}{\delta}+\|R\|_{\rho}\right) \leq \frac{1}{2}
$$

Therefore, we obtain (77) for the functions $\left\{A_{i}^{+}\right\}_{i=1, \ldots, 4}$. 
Proof of Proposition 5.1. We organize the proof of this iterative procedure in three parts. In part I), we correct the invariance of the torus by approximately solving the linearized equation $\mathcal{R}\left(\Delta_{\tau}\right)=-e$, given by (17), as it was explained in Proposition 4.4. Next, in part II) we correct the reducibility of the torus by approximately solving the linearized equation $\mathcal{S}\left(\Delta_{N}, \Delta_{\Lambda}\right)=$ $-\hat{R}$, given by (15) and (18), as it was explained in Proposition 4.6. Finally, in part III) we compute some additional estimates regarding the non-degeneracy conditions for the new torus.

Firstly, let us observe that condition (51) implies condition (75) in Proposition 5.3 by taking a constant $\bar{\alpha}$ larger than $\hat{\alpha}$. Then, we use Proposition 5.3 construct an approximately symplectic basis at every point of the torus. As before, we redefine (enlarge) the constant $\bar{\alpha}$ along the proof to meet the different conditions given in the statement.

I) Correction of the torus: The idea is that the solution of the equation $\mathcal{R}\left(\Delta_{\tau}\right)=-e$ obtained in the invariant and reducible case — as discussed in Proposition 4.4- provides an approximate solution in the approximately invariant case. To this end, we consider the function

$$
\Delta_{\tau}=D \tau \Delta_{1}+V \Delta_{2}+N_{1} \Delta_{3}+N_{2} \Delta_{4}
$$

where $\Delta_{i}$, for $i=1, \ldots, 4$, are solutions of the cohomological equations (37)-(40), taking $\left[\Delta_{1}\right]_{\mathbb{T}^{r}}=0$ and $\left[\Delta_{2}\right]_{\mathbb{T}^{r}}$ given by (41). Then we claim that the new embedding $\bar{\tau}=\tau+\Delta_{\tau}$ parameterizes an approximate reducible and invariant torus $\overline{\mathscr{T}}$ with an error which is quadratic in $\|e\|_{\rho}$ and $\|R\|_{\rho}$. Of course, first we have to check the compatibility condition $\left[D \tau^{\top} J e\right]_{\mathbb{T}^{r}}=$ 0 , that follows from the next computation

$$
D \tau^{\top} J e=D \tau^{\top} J\left(L_{\omega} \tau-J \operatorname{grad} h(\tau)\right)=\Omega_{D \tau} \omega+\operatorname{grad}_{\theta}(h(\tau)),
$$

by observing that both terms at the right hand side have zero average (see Remark 2.2). It is important to observe that $\Delta_{3}^{*}=\mathrm{i} \Delta_{4}$ so the correction $\Delta_{\tau}$ is real analytic.

As far as the estimates are concerned, we have (using Lemma 5.2 to control the solution of the cohomological equations)

$$
\left\|\Delta_{1}\right\|_{\rho-2 \delta} \leq \frac{\bar{\alpha}}{\gamma^{2} \delta^{2 \nu}}\|e\|_{\rho}, \quad\left\|\Delta_{i}\right\|_{\rho-\delta} \leq \frac{\bar{\alpha}}{\gamma \delta^{\nu}}\|e\|_{\rho},
$$

for $i=2,3,4$, so we can control the correction $\Delta_{\tau}$ in the parameterization as follows

$$
\left\|\Delta_{\tau}\right\|_{\rho-2 \delta} \leq \frac{\bar{\alpha}}{\gamma^{2} \delta^{2 \nu}}\|e\|_{\rho}
$$

thus obtaining estimate (56). Moreover, we observe that the derivative of the new parameterization can be controlled easily as follows

$$
\|D \bar{\tau}\|_{\rho-3 \delta} \leq\|D \tau\|_{\rho}+\left\|D \Delta_{\tau}\right\|_{\rho-3 \delta} \leq\|D \tau\|_{\rho}+\frac{\bar{\alpha}}{\gamma^{2} \delta^{2 \nu+1}}\|e\|_{\rho}<\sigma_{1},
$$


where we used hypothesis (51), and also the distance of $\bar{\tau}(\Delta(\rho-2 \delta))$ to the boundary of $\mathcal{U}$

$$
\begin{aligned}
\operatorname{dist}(\bar{\tau}(\Delta(\rho-2 \delta)), \partial \mathcal{U}) & \geq \operatorname{dist}(\tau(\Delta(\rho)), \partial \mathcal{U})-\left\|\Delta_{\tau}\right\|_{\rho-2 \delta} \\
& \geq \operatorname{dist}(\tau(\Delta(\rho)), \partial \mathcal{U})-\frac{\bar{\alpha}}{\gamma^{2} \delta^{2 \nu}}\|e\|_{\rho}>\sigma_{2},
\end{aligned}
$$

where we used hypothesis (52). Notice that we have achieved part of (64) and (65).

Next we control the new error in the invariance. To this end, we first introduce $\Delta_{\tau}$ into $\mathcal{R}\left(\Delta_{\tau}\right)+e$ and we use properties (76) and (84) of the operator $\mathcal{R}$ and also the cohomological equations (37)-(40), thus obtaining

$$
\begin{aligned}
\mathcal{R}\left(\Delta_{\tau}\right)+e= & \mathcal{R}(D \tau) \Delta_{1}+\mathcal{R}(V) \Delta_{2}+\mathcal{R}\left(N_{1}\right) \Delta_{3}+\mathcal{R}\left(N_{2}\right) \Delta_{4} \\
& +D \tau L_{\omega} \Delta_{1}+V L_{\omega} \Delta_{2}+N_{1} L_{\omega} \Delta_{3}+N_{2} L_{\omega} \Delta_{4}+e \\
= & D e \Delta_{1}+\left(D \tau A_{1}^{+}+V A_{2}^{+}+N_{1} A_{3}^{+}+N_{2} A_{4}^{+}\right) \Delta_{2}+R \Delta_{3}+\mathrm{i} R^{*} \Delta_{4} \\
& \underbrace{-D \tau V^{\top} J e+V D \tau^{\top} J e-N_{1} N_{2}^{\top} J e+N_{2} N_{1}^{\top} J e+e}_{e^{+}}
\end{aligned}
$$

We note that the terms not included in $e^{+}$are clearly quadratic in $e$ and $R$, since the functions $\left\{A_{i}^{+}\right\}_{i=1, \ldots, 4}$ and $\left\{\Delta_{i}\right\}_{i=1, \ldots, 4}$ are controlled by $\|e\|_{\rho}$ and $\|R\|_{\rho}$. Then, it suffices to study the remaining part $e^{+}$. To this end, we write $e^{+}$in terms of the constructed basis

$$
e^{+}=D \tau e_{1}^{+}+V e_{2}^{+}+N_{1} e_{3}^{+}+N_{2} e_{4}^{+},
$$

and obtain $\left\{e_{i}^{+}\right\}_{i=1, \ldots, 4}$ by multiplying at both sides by $V^{\top} J, D \tau^{\top} J, N_{2}^{\top} J$ and $N_{1}^{\top} J$. This leads to study the linear system

$$
\left(\mathrm{Id}+M_{3}\right)\left(\begin{array}{c}
e_{1}^{+} \\
e_{2}^{+} \\
e_{3}^{+} \\
e_{4}^{+}
\end{array}\right)=\left(\begin{array}{c}
D_{1} \\
D_{2} \\
D_{3} \\
D_{4}
\end{array}\right),
$$

where $M_{3}$ is given in (86) and the matrices in the right-hand side are the following

$$
\begin{aligned}
& D_{1}=-\left(\Omega_{V, D \tau}-\operatorname{Id}_{r}\right) V^{\top} J e+\Omega_{V} D \tau^{\top} J e-\Omega_{V, N_{1}} N_{2}^{\top} J e+\Omega_{V, N_{2}} N_{1}^{\top} J e, \\
& D_{2}=\Omega_{D \tau} V^{\top} J e-\left(\Omega_{D \tau, V}+\operatorname{Id}_{r}\right) D \tau^{\top} J e+\Omega_{D \tau, N_{1}} N_{2}^{\top} J e-\Omega_{D \tau, N_{2}} N_{1}^{\top} J e, \\
& D_{3}=-\Omega_{N_{2}, D \tau} V^{\top} J e+\Omega_{N_{2}, V} D \tau^{\top} J e-\left(\Omega_{N_{2}, N_{1}}-\operatorname{Id}_{n-r}\right) N_{2}^{\top} J e+\Omega_{N_{2}} N_{1}^{\top} J e, \\
& D_{4}=-\mathrm{i} D_{3}^{*} .
\end{aligned}
$$

Now we control these functions using estimates (68)-(74) in Proposition 5.3

$$
\left\|D_{i}\right\|_{\rho-2 \delta} \leq \frac{\bar{\alpha}}{\gamma \delta^{\nu}}\left(\frac{\|e\|_{\rho}}{\delta}+\|R\|_{\rho}\right)\|e\|_{\rho}
$$


for $i=1, \ldots, 4$. We have shown in the proof of Proposition 5.3 that the matrix $\mathrm{Id}+M_{3}$ is invertible and that $\left\|\left(\mathrm{Id}+M_{3}\right)^{-1}\right\|_{\rho-2 \delta} \leq 2$ (see (92)) so we conclude that

$$
\left\|e^{+}\right\|_{\rho-2 \delta} \leq \frac{\bar{\alpha}}{\gamma \delta^{\nu}}\left(\frac{\|e\|_{\rho}}{\delta}+\|R\|_{\rho}\right)\|e\|_{\rho} .
$$

Going back to equation (94) we get

$$
\left\|\mathcal{R}\left(\Delta_{\tau}\right)+e\right\|_{\rho-3 \delta} \leq \frac{\bar{\alpha}}{\gamma^{2} \delta^{2 \nu+1}}\left(\frac{\|e\|_{\rho}}{\delta}+\|R\|_{\rho}\right)\|e\|_{\rho}
$$

and therefore, we conclude that $\mathcal{R}\left(\Delta_{\tau}\right)=-e$ is solved modulo quadratic terms in the errors. Then, we observe that

$$
\begin{aligned}
\bar{e} & =L_{\omega} \bar{\tau}-J \operatorname{grad} h(\bar{\tau}) \\
& =\mathcal{R}\left(\Delta_{\tau}\right)+e+J\left(\operatorname{grad} h(\tau)+\operatorname{hess} h(\tau) \Delta_{\tau}-\operatorname{grad} h\left(\tau+\Delta_{\tau}\right)\right)
\end{aligned}
$$

and control the last terms by estimating the residue of the Taylor expansion of $h$ up to second order, thus obtaining

$$
\left\|\operatorname{grad} h(\tau)+\operatorname{hess} h(\tau) \Delta_{\tau}-\operatorname{grad} h\left(\tau+\Delta_{\tau}\right)\right\|_{\rho-2 \delta} \leq \frac{\bar{\alpha}}{\gamma^{4} \delta^{4 \nu}}\|e\|_{\rho}^{2} .
$$

Hence, we end up with

$$
\|\bar{e}\|_{\rho-3 \delta} \leq \frac{\bar{\alpha}}{\gamma^{4} \delta^{4 \nu-1}}\left(\frac{\|e\|_{\rho}}{\delta}+\|R\|_{\rho}\right)\|e\|_{\rho},
$$

where we used that $\nu>r-1 \geq 1$, finally obtaining estimate (57).

II) Correction of the reducibility: To square the error in reducibility of the new torus $\overline{\mathscr{T}}$ we have to deal with the equation $\mathcal{S}\left(\Delta_{N}, \Delta_{\Lambda}\right)=-\hat{R}$, given by (15) and (18). As before, we solve approximately this equation by taking (the reason of writing $\hat{\Delta}_{N}$ rather than $\Delta_{N}$ will be clear later on)

$$
\hat{\Delta}_{N}=D \tau P_{1}+V P_{2}+N_{1} P_{3}+N_{2} P_{4},
$$

$\left\{P_{i}\right\}_{i=1, \ldots, 4}$ and $\Delta_{\Lambda}$ being the solution of the cohomological equations (42)-(45) for

$$
\hat{R}=R+J \operatorname{hess} h(\tau) N-J \operatorname{hess} h(\bar{\tau}) N,
$$

and fixing diag $\left[P_{3}\right]_{\mathbb{T}^{r}}=0$. The formal solution of these equation has been discussed in Proposition 4.6 so we know that we must take $\Delta_{\Lambda}=-\operatorname{diag}\left[N_{2}^{\top} J \hat{R}\right]_{\mathbb{T}^{r}}$.

Firstly, we claim that the geometry of the problem imposes that the selected $\Delta_{\Lambda}$ is pure imaginary, so our procedure automatically preserves the approximately elliptic character of the torus. To see that, we observe that transposing equation (83) leads to

$$
L_{\omega} \Omega_{N_{1}, N_{2}}=-\Lambda \Omega_{N_{1}, N_{2}}+\Omega_{N_{1}, N_{2}} \Lambda+\Omega_{R, N_{2}}+\mathrm{i} \Omega_{N_{1}, R^{*}} .
$$


Since the left-hand side of this expression has vanishing average and diag $\left[\Omega_{N_{1}, N_{2}}\right]_{\mathbb{T}^{r}}=-\operatorname{Id}_{n-r}$, it turns out that

$$
\operatorname{diag}\left[\Omega_{R, N_{2}}+\mathrm{i} \Omega_{N_{1}, R^{*}}\right]_{\mathbb{T}^{r}}=0,
$$

and so diag $\left[\mathrm{i} \Omega_{N_{1}, R^{*}}\right]_{\mathbb{T}^{r}}=\operatorname{diag}\left[\Omega_{N_{2}, R}\right]_{\mathbb{T}^{r}}^{\top}$. Then, it is straightforward to compute

$$
\begin{aligned}
\Delta_{\Lambda}^{*} & =-\operatorname{diag}\left[N_{2}^{\top} J \hat{R}\right]_{\mathbb{T}^{r}}^{*}=-\operatorname{diag}\left[-\mathrm{i} \Omega_{N_{1}, R^{*}}+N_{1}^{\top}(\operatorname{hess} h(\tau)-\operatorname{hess} h(\bar{\tau})) N_{2}\right]_{\mathbb{T}^{r}} \\
& =\operatorname{diag}\left[\Omega_{N_{2}, R}\right]_{\mathbb{T}^{r}}^{\top}-\operatorname{diag}\left[N_{2}^{\top}(\operatorname{hess} h(\tau)-\operatorname{hess} h(\bar{\tau})) N_{1}\right]_{\mathbb{T}^{r}}^{\top} \\
& =\operatorname{diag}\left[\Omega_{N_{2}, R}-N_{2}^{\top}(\operatorname{hess} h(\tau)-\operatorname{hess} h(\bar{\tau})) N_{1}\right]_{\mathbb{T}^{r}}^{\top}=-\Delta_{\Lambda}^{\top}=-\Delta_{\Lambda},
\end{aligned}
$$

so $\Delta_{\Lambda}$ is pure imaginary.

Now obtaining estimates for the solution of the cohomological equations is straightforward after controlling

$$
\|\hat{R}\|_{\rho-2 \delta} \leq \frac{\bar{\alpha}}{\gamma^{2} \delta^{2 \nu-1}}\left(\frac{\|e\|_{\rho}}{\delta}+\|R\|_{\rho}\right), \quad\left|\Delta_{\Lambda}\right| \leq \frac{\bar{\alpha}}{\gamma^{2} \delta^{2 \nu-1}}\left(\frac{\|e\|_{\rho}}{\delta}+\|R\|_{\rho}\right),
$$

and applying Lemma 5.2

$$
\left\|P_{1}\right\|_{\rho-4 \delta} \leq \frac{\bar{\alpha}}{\gamma^{4} \delta^{4 \nu-1}}\left(\frac{\|e\|_{\rho}}{\delta}+\|R\|_{\rho}\right), \quad\left\|P_{i}\right\|_{\rho-3 \delta} \leq \frac{\bar{\alpha}}{\gamma^{3} \delta^{3 \nu-1}}\left(\frac{\|e\|_{\rho}}{\delta}+\|R\|_{\rho}\right)
$$

for $i=2,3,4$. With these estimates we check condition (64) for the new approximate normal frequencies $\bar{\lambda}$. For example,

$$
\left|\bar{\lambda}_{i} \pm \bar{\lambda}_{j}\right| \geq\left|\lambda_{i} \pm \lambda_{j}\right|-2\left|\Delta_{\Lambda}\right| \geq \min _{i \neq j}\left|\lambda_{i} \pm \lambda_{j}\right|-2\left|\Delta_{\Lambda}\right|>\sigma_{3}
$$

where we used (53). Similar computations allow us to see that $\frac{\sigma_{3}}{2}<\left|\bar{\lambda}_{j}\right|<\frac{\sigma_{4}}{2}$, using (54) and (55), respectively.

We also have

$$
\left\|\hat{\Delta}_{N}\right\|_{\rho-4 \delta} \leq \frac{\bar{\alpha}}{\gamma^{4} \delta^{4 \nu-1}}\left(\frac{\|e\|_{\rho}}{\delta}+\|R\|_{\rho}\right)
$$

and we observe that, if we introduce $\hat{N}=N+\hat{\Delta}_{N}$, using (51) we obtain that

$$
\|\hat{N}\|_{\rho-4 \delta} \leq\|N\|_{\rho}+\left\|\hat{\Delta}_{N}\right\|_{\rho-4 \delta} \leq\|N\|_{\rho}+\frac{\bar{\alpha}}{\gamma^{4} \delta^{4 \nu-1}}\left(\frac{\|e\|_{\rho}}{\delta}+\|R\|_{\rho}\right)<\sigma_{1},
$$

and that the matrix $\operatorname{diag}\left[\Omega_{\hat{N}, \hat{N}^{*}}\right]_{\mathbb{T}^{r}}$ is constant, diagonal and pure imaginary, but it is not $\operatorname{idd}_{n-r}$ as we want. Nevertheles, from the following expression

$$
\Omega_{\hat{N}, \hat{N}^{*}}-\Omega_{N, N^{*}}=\Omega_{N, \hat{\Delta}_{N}^{*}}+\Omega_{\hat{\Delta}_{N}, \hat{N}^{*}}
$$


and using hypothesis (51) we obtain

$$
\left\|\Omega_{\hat{N}, \hat{N}^{*}}-\Omega_{N, N^{*}}\right\|_{\rho-4 \delta} \leq \frac{\bar{\alpha}}{\gamma^{4} \delta^{4 \nu-1}}\left(\frac{\|e\|_{\rho}}{\delta}+\|R\|_{\rho}\right)
$$

where we recall that diag $\left[\Omega_{N, N^{*}}\right]_{\mathbb{T}^{r}}=\mathrm{i} \operatorname{Id}_{n-r}$. Hence, we have that the elements of $\operatorname{diag}\left[\Omega_{\hat{N}, \hat{N}^{*}}\right]_{\mathbb{T}^{r}}$ are of the form $\mathrm{i}\left(1+d_{i}\right)$ with

$$
\left|d_{i}\right| \leq \frac{\bar{\alpha}}{\gamma^{4} \delta^{4 \nu-1}}\left(\frac{\|e\|_{\rho}}{\delta}+\|R\|_{\rho}\right)
$$

Hence, using again hypothesis (51), we have that $\left|d_{i}\right| \leq 1 / 2$ for $i=1, \ldots, n-r$, so we can normalize $\hat{N}$ in order to preserve hypothesis $\mathrm{H}_{3}$. To this end, we define the real matrix

$$
B=\operatorname{diag}\left(b_{1}, \ldots, b_{n-r}\right), \quad \text { with } \quad b_{i}=\sqrt{\frac{1}{1+d_{i}}}
$$

and it turns out that the matrix $\bar{N}=\hat{N} B$ satisfies $\operatorname{diag}\left[\Omega_{\bar{N}, \bar{N}^{*}}\right]_{\mathbb{T}^{r}}=\mathrm{iId}_{n-r}$. Let us observe that the performed correction is small, since if we take $\bar{N}=N+\Delta_{N}$ we have that

$$
\Delta_{N}=N\left(B-\operatorname{Id}_{n-r}\right)+\hat{\Delta}_{N} B
$$

and so

$$
\left\|\Delta_{N}\right\|_{\rho-4 \delta} \leq \frac{\bar{\alpha}}{\gamma^{4} \delta^{4 \nu-1}}\left(\frac{\|e\|_{\rho}}{\delta}+\|R\|_{\rho}\right),
$$

that corresponds to estimate (59). We see that $\|\bar{N}\|_{\rho-4 \delta}<\sigma_{1}$ by similar computations as in (97), thus obtaining the corresponding condition in (65).

The rest of this part is devoted to check that, using $\bar{N}$ and $\bar{\Lambda}$, the new approximately invariant torus $\overline{\mathscr{T}}$ is approximately elliptic up to a quadratic error. To this end, we compute

$$
\begin{aligned}
\bar{R} & =L_{\omega} \bar{N}-J \text { hess } h(\bar{\tau}) \bar{N}+\bar{N} \bar{\Lambda} \\
& =\mathcal{S}\left(\Delta_{N}, \Delta_{\Lambda}\right)+\hat{R}+J(\operatorname{hess} h(\tau)-\operatorname{hess} h(\bar{\tau})) \Delta_{N}+\Delta_{N} \Delta_{\Lambda},
\end{aligned}
$$

where the action of $\mathcal{S}$ on $\Delta_{N}$ is written in terms of the action on $\hat{\Delta}_{N}$ as follows

$$
\begin{aligned}
\mathcal{S}\left(\Delta_{N}, \Delta_{\Lambda}\right) & +\hat{R} \\
= & \mathcal{S}\left(N\left(B-\operatorname{Id}_{n-r}\right)+\hat{\Delta}_{N} B, \Delta_{\Lambda}\right)+\hat{R} \\
= & \mathcal{R}\left(N\left(B-\operatorname{Id}_{n-r}\right)\right)+\mathcal{R}\left(\hat{\Delta}_{N} B\right)+N \Delta_{\Lambda}+N\left(B-\operatorname{Id}_{n-r}\right) \Lambda+\hat{\Delta}_{N} B \Lambda+\hat{R} \\
= & R\left(B-\operatorname{Id}_{n-r}\right)+\mathcal{R}\left(\hat{\Delta}_{N}\right) B+N \Delta_{\Lambda}+\hat{\Delta}_{N} B \Delta+\hat{R} \\
= & \left(\mathcal{S}\left(\hat{\Delta}_{N}, \Delta_{\Lambda}\right)+\hat{R}\right) B+\left(R-\hat{R}-N \Delta_{\Lambda}+\hat{\Delta}_{N} \Lambda\right)\left(B-\operatorname{Id}_{n-r}\right),
\end{aligned}
$$


where we used that $\mathcal{R}(N)=-N \Lambda+R$ and $B \Lambda=\Lambda B$.

Then, we introduce $\hat{\Delta}_{N}$ and $\Delta_{\Lambda}$ in $\mathcal{S}\left(\hat{\Delta}_{N}, \Delta_{\Lambda}\right)+\hat{R}$ and we use the properties (76) and (84) of the operator $\mathcal{R}$ and also the cohomological equations (42)-(45), thus obtaining

$$
\begin{aligned}
\mathcal{S}\left(\hat{\Delta}_{N}, \Delta_{\Lambda}\right)+\hat{R}= & \mathcal{R}\left(\hat{\Delta}_{N}\right)+N_{1} \Delta_{\Lambda}+\hat{\Delta}_{N} \Lambda+\hat{R} \\
= & \mathcal{R}(D \tau) P_{1}+\mathcal{R}(V) P_{2}+\mathcal{R}\left(N_{1}\right) P_{3}+\mathcal{R}\left(N_{2}\right) P_{4} \\
& +D \tau L_{\omega} P_{1}+V L_{\omega} P_{2}+N_{1} L_{\omega} P_{3}+N_{2} L_{\omega} P_{4}+N_{1} \Delta_{\Lambda} \\
& +D \tau P_{1} \Lambda+V P_{2} \Lambda+N_{1} P_{3} \Lambda+N_{2} P_{4} \Lambda+\hat{R} \\
= & D e P_{1}+\left(D \tau A_{1}^{+}+V A_{2}^{+}+N_{1} A_{3}^{+}+N_{2} A_{4}^{+}\right) P_{2}+R P_{3}+\mathrm{i} R^{*} P_{4} \\
& \underbrace{-D \tau V^{\top} J \hat{R}+V D \tau^{\top} J \hat{R}-N_{1} N_{2}^{\top} J \hat{R}+N_{2} N_{1}^{\top} J \hat{R}+\hat{R}}_{R^{+}} .
\end{aligned}
$$

As we made in equation (94), the terms not included in $R^{+}$are clearly quadratic in $e$ and $R$. Then, we express $R^{+}$in terms of the basis

$$
R^{+}=D \tau R_{1}^{+}+V R_{2}^{+}+N_{1} R_{3}^{+}+N_{2} R_{4}^{+},
$$

and for $R_{j}^{+}$we get a system like (95) for $e_{j}^{+}$, simply by replacing $e$ with $\hat{R}$ in the definition of $D_{j}$. Hence,

$$
\left\|R^{+}\right\|_{\rho-2 \delta} \leq \frac{\bar{\alpha}}{\gamma^{3} \delta^{3 \nu-1}}\left(\frac{\|e\|_{\rho}}{\delta}+\|R\|_{\rho}\right)^{2}
$$

Therefore, we can compute a bound for the error in the solution of the linear equation that corrects reducibility

$$
\left\|\mathcal{S}\left(\hat{\Delta}_{N}, \Delta_{\Lambda}\right)+\hat{R}\right\|_{\rho-4 \delta} \leq \frac{\bar{\alpha}}{\gamma^{4} \delta^{4 \nu}}\left(\frac{\|e\|_{\rho}}{\delta}+\|R\|_{\rho}\right)^{2},
$$

so we obtain - again, we use hypothesis (51) to control the quadratic terms-

$$
\left\|\mathcal{S}\left(\Delta_{N}, \Delta_{\Lambda}\right)+\hat{R}\right\|_{\rho-4 \delta} \leq \frac{\bar{\alpha}}{\gamma^{8} \delta^{8 \nu-2}}\left(\frac{\|e\|_{\rho}}{\delta}+\|R\|_{\rho}\right)^{2} .
$$

Therefore, recalling (98), we easily show that the new error (60) in reducibility is quadratic

$$
\|\bar{R}\|_{\rho-4 \delta} \leq \frac{\bar{\alpha}}{\gamma^{8} \delta^{8 \nu-2}}\left(\frac{\|e\|_{\rho}}{\delta}+\|R\|_{\rho}\right)^{2} .
$$

III) Additional estimates: Finally, we have to check estimates that allow us to control the non-degeneracy of the basis and the twist condition. Using that

$$
G_{D \bar{\tau}}-G_{D \tau}=G_{D \tau, D \Delta_{\tau}}+G_{D \Delta_{\tau}, D \bar{\tau}}
$$


and recalling (51) and (56), we get

$$
\left\|G_{D \bar{\tau}}-G_{D \tau}\right\|_{\rho-3 \delta} \leq \frac{\bar{\alpha}}{\gamma^{2} \delta^{2 \nu+1}}\|e\|_{\rho}
$$

Now, we observe that $G_{D \bar{\tau}}^{-1}=\left(\operatorname{Id}_{r}+G_{D \tau}^{-1}\left(G_{D \bar{\tau}}-G_{D \tau}\right)\right)^{-1} G_{D \tau}^{-1}$ so we can compute the following —again, we make use of (51) —

$$
\begin{aligned}
\left\|G_{D \bar{\tau}}^{-1}-G_{D \tau}^{-1}\right\|_{\rho-3 \delta} & \leq\left\|G_{D \tau}^{-1}\right\|_{\rho}\left\|\left(\operatorname{Id}_{r}+G_{D \tau}^{-1}\left(G_{D \bar{\tau}}-G_{D \tau}\right)\right)^{-1}-\operatorname{Id}_{r}\right\|_{\rho-3 \delta} \\
& \leq \frac{\left\|G_{D \tau}^{-1}\right\|_{\rho}^{2}\left\|G_{D \bar{\tau}}-G_{D \tau}\right\|_{\rho-3 \delta}}{1-\left\|G_{D \tau}^{-1}\right\|_{\rho}\left\|G_{D \bar{\tau}}-G_{D \tau}\right\|_{\rho-3 \delta}} \leq \frac{\bar{\alpha}}{\gamma^{2} \delta^{2 \nu+1}}\|e\|_{\rho}
\end{aligned}
$$

thus obtaining (61) and the term in (65) that corresponds to $G_{D \bar{\tau}}^{-1}$. Similar computations allow us to control the non-degeneracy of the set of normal vectors, thus getting (62) and (65) for $G_{\bar{N}, \bar{N}^{*}}^{-1}$. Now, we are able to estimate the new twist condition for

$$
\bar{A}_{1}(\theta)=G_{D \bar{\tau}}^{-1}(\theta) D \bar{\tau}(\theta)^{\top}\left(\bar{T}_{1}(\theta)+\bar{T}_{2}(\theta)+\bar{T}_{2}(\theta)^{\top}\right) D \bar{\tau}(\theta) G_{D \bar{\tau}}^{-1}(\theta)
$$

where

$$
\begin{aligned}
& \bar{T}_{1}(\theta)=J^{\top} \operatorname{hess} h(\bar{\tau}(\theta)) J-\operatorname{hess} h(\bar{\tau}(\theta)), \\
& \bar{T}_{2}(\theta)=\bar{T}_{1} J\left[D \bar{\tau}(\theta) G_{D \bar{\tau}}(\theta)^{-1} D \bar{\tau}(\theta)^{\top}-\mathrm{Id}\right] \operatorname{Re}\left(\mathrm{i} \bar{N}(\theta) \bar{N}^{*}(\theta)^{\top}\right) .
\end{aligned}
$$

As before, we first bound

$$
\left|\left[\bar{A}_{1}\right]_{\mathbb{T}^{r}}-\left[A_{1}\right]_{\mathbb{T}^{r}}\right| \leq \frac{\bar{\alpha}}{\gamma^{4} \delta^{4 \nu-1}}\left(\frac{\|e\|_{\rho}}{\delta}+\|R\|_{\rho}\right) .
$$

Now we estimate the inverse of $\left[\bar{A}_{1}\right]_{\mathbb{T}^{r}}$ by using the fact that $\bar{A}_{1}=A_{1}+\bar{A}_{1}-A_{1}$. Then, we repeat the same argument used before, using hypothesis (51), thus obtaining bounds (63) and (65) for $\left[\bar{A}_{1}\right]_{\mathbb{T}^{r}}^{-1}$.

\section{Proof of the main result}

In this section we prove Theorem 3.1 by applying inductively Proposition 5.1. First, in Section 6.1 we study the convergence of the obtained iterative scheme, without worrying about the exclusion of parameters that lead to resonances. As usual, the quadratic convergence overcomes the effect of small divisors. Then, in Section 6.2 we prove that Lipschitz regularity is preserved along the iterative procedure. Finally, in Section 6.3, we estimate the measure of the set of excluded parameters. 


\subsection{Convergence of the Newton scheme}

Given a parameter $\mu \in I$, we denote the objects that characterize the corresponding approximately elliptic and invariant torus as (from now on we omit the dependence on the parameter)

$$
\tau_{(0)}=\tau_{\mu}, \quad N_{(0)}=N_{\mu}, \quad \Lambda_{(0)}=\Lambda_{\mu},
$$

and we introduce also

$$
e_{(0)}=e_{\mu}, \quad R_{(0)}=R_{\mu}, \quad A_{1,(0)}=A_{1, \mu}, \quad \lambda_{(0)}=\left(\lambda_{1}^{(0)}, \ldots, \lambda_{n-r}^{(0)}\right)=\lambda_{\mu} .
$$

where we recall that $\Lambda_{(0)}=\operatorname{diag}\left(\mathrm{i} \lambda_{(0)}\right)$. Moreover, given $\gamma_{0}>0$ such that $\gamma_{0} \leq \frac{1}{2} \min \{1, \hat{\gamma}\}$, we define the following quantities (recall that $0<\rho<1$ )

$$
\rho_{(0)}=\rho, \quad \delta_{(0)}=\frac{\rho_{(0)}}{16}, \quad \rho_{(s)}=\rho_{(s-1)}-4 \delta_{(s-1)}, \quad \delta_{(s)}=\frac{\delta_{(0)}}{2^{s}}, \quad \gamma_{(s)}=\left(1+2^{-s}\right) \gamma_{0},
$$

for any $s \geq 1$, and consider the normalized error

$$
\varepsilon_{(0)}=\frac{\left\|e_{(0)}\right\|_{\rho_{(0)}}}{\delta_{(0)}}+\left\|R_{(0)}\right\|_{\rho_{(0)}} .
$$

Then, we are going to show that, considering the constant $\bar{\alpha}$ provided by Proposition 5.1, which depends on the quantities $\nu, r, n,|\omega|, \sigma_{0}, \sigma_{1}, \sigma_{2}, \sigma_{3}$ and $\sigma_{4}$ in the statement of Theorem 3.1, if the normalized error $\varepsilon_{(0)}$ is sufficiently small so that

$$
\frac{2^{8 \nu-1} \bar{\alpha} \varepsilon_{(0)}}{\gamma_{0}^{8} \delta_{(0)}^{8 \nu-2}}<\frac{1}{2} \min \left\{1, \sigma_{1}-\sigma^{*}, \operatorname{dist}\left(\tau_{(0)}(\Delta(\rho)), \partial \mathcal{U}\right)-\sigma_{2}, \sigma^{* *}-\sigma_{3}, \sigma_{4}-2 \max _{j}\left|\lambda_{j}^{(0)}\right|\right\},
$$

where

$$
\begin{aligned}
\sigma^{*} & =\max \left\{\left\|D \tau_{(0)}\right\|_{\rho_{(0)}},\left\|N_{(0)}\right\|_{\rho_{(0)}},\left\|G_{D \tau_{(0)}}^{-1}\right\|_{\rho_{(0)}},\left\|G_{N_{(0)}, N_{(0)}^{*}}^{-1}\right\|_{\rho_{(0)}},\left|\left[A_{1,(0)}\right]_{\mathbb{T}^{r}}^{-1}\right|\right\}, \\
\sigma^{* *} & =\min \left\{\min _{i \neq j}\left|\lambda_{i}^{(0)} \pm \lambda_{j}^{(0)}\right|, 2 \min _{j}\left|\lambda_{j}^{(0)}\right|\right\} .
\end{aligned}
$$

then we can apply recursively Proposition 5.1 to the initial approximation, thus obtaining a sequence

$$
\begin{aligned}
\tau_{(s)} & =\bar{\tau}_{(s-1)}=\tau_{(s-1)}+\Delta_{\tau_{(s-1)}}, & e_{(s)} & =\bar{e}_{(s-1)}, \\
N_{(s)} & =\bar{N}_{(s-1)}=N_{(s-1)}+\Delta_{N_{(s-1)}}, & R_{(s)} & =\bar{R}_{(s-1)}, \\
\Lambda_{(s)} & =\bar{\Lambda}_{(s-1)}=\Lambda_{(s-1)}+\Delta_{\Lambda_{(s-1)}}, & A_{1,(s)} & =\bar{A}_{1,(s-1)},
\end{aligned}
$$


all these objects being analytic in $\Delta\left(\rho_{(s)}\right)$. Notice that, in order to apply $s$ times Proposition 5.1, we restrict the parameter $\mu$ to the set $I_{(s-1)}$ defined iteratively by $I_{(-1)}=I$ and

$$
\begin{aligned}
I_{(s)}=\left\{\mu \in I_{(s-1)}:\right. & \lambda_{(s)} \text { satisfies Diophantine conditions (9) } \\
& \text { of } \left.\left(\gamma_{(s)}, \nu\right) \text {-type with respect to } \omega\right\} .
\end{aligned}
$$

Let us observe that the basic frequencies $\omega$ automatically satisfy Diophantine conditions (4) of $\left(\gamma_{(s)}, \nu\right)$-type, for every $s \geq 0$, since they are fixed along the procedure and we have $\gamma_{(s)} \leq$ $2 \gamma_{0} \leq \hat{\gamma}$ and $\nu>\hat{\nu}$.

Now we proceed by induction. We suppose that we have applied $s$ times Proposition 5.1, for certain $s \geq 0$, and we verify that we can apply it again. To this end, we define $\varepsilon_{(s)}, \sigma_{(s)}^{*}$ and $\sigma_{(s)}^{* *}$ as in (99), (101) and (102), just by replacing the (0)-objects with $(s)$-ones. First, we observe that we have

$$
\sigma_{(s)}^{*}<\sigma_{1}, \quad \operatorname{dist}\left(\tau_{(s)}\left(\Delta\left(\rho_{(s)}\right)\right), \partial \mathcal{U}\right)>\sigma_{2}, \quad \sigma_{(s)}^{* *}>\sigma_{3}, \quad \max _{j}\left|\lambda_{j}^{(s)}\right|<\frac{\sigma_{4}}{2},
$$

so the construction of the constant $\bar{\alpha}$ of Proposition 5.1 is uniform for all iterative steps -it depends on the constants $\sigma_{0}, \sigma_{1}, \sigma_{2}, \sigma_{3}$ and $\sigma_{4}$ that remain unchanged along the procedureand so, conditions (51)-(55) are fullfilled provided that the normalized error $\varepsilon_{(s)}$ satisfies

$$
\frac{\bar{\alpha} \varepsilon_{(s)}}{\gamma_{(s)}^{4} \delta_{(s)}^{4 \nu-1}}<\frac{1}{2} \min \left\{1, \sigma_{1}-\sigma_{(s)}^{*}, \operatorname{dist}\left(\tau_{(s)}\left(\Delta\left(\rho_{(s)}\right)\right), \partial \mathcal{U}\right)-\sigma_{2}, \sigma_{(s)}^{* *}-\sigma_{3}, \sigma_{4}-2 \max _{j}\left|\lambda_{j}^{(s)}\right|\right\}
$$

In order to verify this inequality, we start by computing the normalized error at the $s$-th step -recall that $\gamma_{(s)}<1$ and $\delta_{(s)}<1-$

$$
\varepsilon_{(s)}=\frac{\left\|e_{(s)}\right\|_{\rho_{(s)}}}{\delta_{(s)}}+\left\|R_{(s)}\right\|_{\rho_{(s)}} \leq \frac{2 \bar{\alpha}}{\gamma_{(s-1)}^{8} \delta_{(s-1)}^{8 \nu-2}} \varepsilon_{(s-1)}^{2} \leq \frac{2^{(s-1)(8 \nu-2)+1} \bar{\alpha}}{\gamma_{0}^{8} \delta_{(0)}^{8 \nu-2}} \varepsilon_{(s-1)}^{2},
$$

where we used (57), (60) and the fact that $\gamma_{(s-1)} \geq \gamma_{0}$. Then, by iterating this sequence backwards, we obtain that

$$
\varepsilon_{(s)} \leq \frac{\gamma_{0}^{8} \delta_{(0)}^{8 \nu-2}}{2 \bar{\alpha}} 2^{-(s+1)(8 \nu-2)}\left(\frac{2^{8 \nu-1} \bar{\alpha} \varepsilon_{(0)}}{\gamma_{0}^{8} \delta_{(0)}^{8 \nu-2}}\right)^{2^{s}}
$$

Using this expression of the error, we verify condition (104) in order to perform the step $s+1$. For example, the first term in this condition is straightforward

$$
\frac{\bar{\alpha} \varepsilon_{(s)}}{\gamma_{(s)}^{4} \delta_{(s)}^{4 \nu-1}} \leq \frac{1}{2} \gamma_{0}^{4} \delta_{(0)}^{4 \nu-1} 2^{-(4 \nu-1) s-8 \nu+2}\left(\frac{2^{8 \nu-1} \bar{\alpha} \varepsilon_{(0)}}{\gamma_{0}^{8} \delta_{(0)}^{8 \nu-2}}\right)^{2^{s}}<\frac{1}{2},
$$


recalling that $\nu>r-1 \geq 1$ and (100). In order to verify the remaining conditions in (104), we have to control also the objects $\left\|D \tau_{(s)}\right\|_{\rho_{(s)}},\left\|N_{(s)}\right\|_{\rho_{(s)}},\left|\Lambda_{(s)}\right|$, etc. For example, we discuss in detail the following inequality

$$
\left\|D \tau_{(s)}\right\|_{\rho_{(s)}}+\frac{\bar{\alpha} \varepsilon_{(s)}}{\gamma_{(s)}^{4} \delta_{(s)}^{4 \nu-1}}<\sigma_{1}
$$

By using $D \tau_{(s)}=D \tau_{(s-1)}+D \Delta_{\tau_{(s-1)}}$ recursively as follows

$$
\begin{aligned}
\left\|D \tau_{(s)}\right\|_{\rho_{(s)}}+\frac{\bar{\alpha} \varepsilon_{(s)}}{\gamma_{(s)}^{4} \delta_{(s)}^{4 \nu-1}} & \leq\left\|D \tau_{(s-1)}\right\|_{\rho_{(s-1)}}+\left\|D \Delta_{\tau_{(s-1)}}\right\|_{\rho_{(s)}}+\frac{\bar{\alpha} \varepsilon_{(s)}}{\gamma_{(s)}^{4} \delta_{(s)}^{4 \nu-1}} \\
& \leq\left\|D \tau_{(0)}\right\|_{\rho_{(0)}}+\sum_{j=0}^{s-1}\left\|D \Delta_{\tau_{(j)}}\right\|_{\rho_{(j+1)}}+\frac{\bar{\alpha} \varepsilon_{(s)}}{\gamma_{(s)}^{4} \delta_{(s)}^{4 \nu-1}} \\
& \leq\left\|D \tau_{(0)}\right\|_{\rho_{(0)}}+\sum_{j=0}^{s-1} \frac{\bar{\alpha} \varepsilon_{(j)}}{\gamma_{(j)}^{2} \delta_{(j)}^{2 \nu}}+\frac{\bar{\alpha} \varepsilon_{(s)}}{\gamma_{(s)}^{4} \delta_{(s)}^{4 \nu-1}} \\
& \leq\left\|D \tau_{(0)}\right\|_{\rho_{(0)}}+\sum_{j=0}^{s} \frac{\bar{\alpha} \varepsilon_{(j)}}{\gamma_{(j)}^{4} \delta_{(j)}^{4 \nu-1}} .
\end{aligned}
$$

Notice that in the above computations we used estimate (56) in Proposition 5.1 and the fact that $\gamma_{(s)}, \delta_{(s)}<1$. Then, we introduce the expression for the errors $\varepsilon_{(j)}$ previously computed and use that $j+1 \leq 2^{j}$ in order to obtain

$$
\begin{aligned}
\left\|D \tau_{(s)}\right\|_{\rho_{(s)}}+\frac{\bar{\alpha} \varepsilon_{(s)}}{\gamma_{(s)}^{4} \delta_{(s)}^{4 \nu-1}} & \leq\left\|D \tau_{(0)}\right\|_{\rho_{(0)}}+\gamma_{0}^{4} \delta_{(0)}^{4 \nu-1} 2^{-8 \nu+1} \sum_{j=0}^{s} 2^{-(4 \nu-1) j}\left(\frac{2^{8 \nu-1} \bar{\alpha} \varepsilon_{(0)}}{\gamma_{0}^{8} \delta_{(0)}^{8 \nu-2}}\right)^{2^{j}} \\
& \leq\left\|D \tau_{(0)}\right\|_{\rho_{(0)}}+\gamma_{0}^{4} \delta_{(0)}^{4 \nu-1} 2^{-8 \nu+1} \sum_{j=0}^{\infty}\left(\frac{2^{8 \nu-1} \bar{\alpha} \varepsilon_{(0)}}{\gamma_{0}^{8} \delta_{(0)}^{8 \nu-2}}\right)^{j+1} \\
& \leq\left\|D \tau_{(0)}\right\|_{\rho_{(0)}}+\frac{2 \bar{\alpha} \varepsilon_{(0)}}{\gamma_{0}^{4} \delta_{(0)}^{4 \nu-1}}<\sigma_{1}
\end{aligned}
$$

where in the last two inequalities we have used hypothesis (100) in order to bound the expression by the sum of a geometric progression of ratio $1 / 2$. Analogous computations show that - we use estimates (59), (61), (62) and (63), respectively-

$$
\begin{array}{cc}
\left\|N_{(s)}\right\|_{\rho_{(s)}}+\frac{\bar{\alpha} \varepsilon_{(s)}}{\gamma_{(s)}^{4} \delta_{(s)}^{4 \nu-1}}<\sigma_{1}, \quad & \left\|G_{D \tau_{(s)}}^{-1}\right\|_{\rho_{(s)}}+\frac{\bar{\alpha} \varepsilon_{(s)}}{\gamma_{(s)}^{4} \delta_{(s)}^{4 \nu-1}}<\sigma_{1}, \\
\left\|G_{N_{(s)}, N_{(s)}^{*}}^{-1}\right\|_{\rho_{(s)}}+\frac{\bar{\alpha} \varepsilon_{(s)}}{\gamma_{(s)}^{4} \delta_{(s)}^{4 \nu-1}}<\sigma_{1}, & \left|\left[A_{(s)}\right]_{\mathbb{T}^{r}}^{-1}\right|+\frac{\bar{\alpha} \varepsilon_{(s)}}{\gamma_{(s)}^{4} \delta_{(s)}^{4 \nu-1}}<\sigma_{1},
\end{array}
$$


thus obtaining the second condition in (104). Next, to verify the inequality which corresponds to the third term in (104) we observe that

$$
\operatorname{dist}\left(\tau_{(s)}\left(\Delta\left(\rho_{(s)}\right)\right), \partial \mathcal{U}\right) \geq \operatorname{dist}\left(\tau_{(s-1)}\left(\Delta\left(\rho_{(s-1)}\right)\right), \partial \mathcal{U}\right)-\left\|\Delta_{\tau_{(s-1)}}\right\|_{\rho_{(s)}},
$$

and we use again (56) and (100), thus concluding — computations are analogous as those performed for $D \tau_{(s)}$ above-

$$
\operatorname{dist}\left(\tau_{(s)}\left(\Delta\left(\rho_{(s)}\right)\right), \partial \mathcal{U}\right)-\frac{\bar{\alpha} \varepsilon_{(s)}}{\gamma_{(s)}^{4} \delta_{(s)}^{4 \nu-1}} \geq \operatorname{dist}\left(\tau_{(0)}\left(\Delta\left(\rho_{(0)}\right)\right), \partial \mathcal{U}\right)-\frac{2 \bar{\alpha} \varepsilon_{(0)}}{\gamma_{0}^{4} \delta_{(0)}^{4 \nu-1}}>\sigma_{2} .
$$

Checking fourth and fifth conditions in (104) —which involves estimates (58) for the normal frequencies - is left to the reader, since it follows in the same way.

We now observe that hypotheses $\mathrm{H}_{1}, \mathrm{H}_{2}, \mathrm{H}_{3}, \mathrm{H}_{4}$ and $\mathrm{H}_{5}$ are automatically satisfied for the $s$-objects and Diophantine conditions in $\mathrm{H}_{6}$ are guaranteed after defining the sets $I_{(s)}$ of "good parameters". Then, we can apply Proposition 5.1 again.

Therefore, we can apply inductively this scheme and, since the sequence of normalized errors satisfies $\varepsilon_{(s)} \rightarrow 0$ as $s \rightarrow \infty$ (due to hypothesis (100)) we converge to a true quasiperiodic invariant torus for every $\mu$ in the set

$$
I_{(\infty)}=\bigcap_{s \geq 0} I_{(s)}
$$

Notice also that

$$
\rho_{(\infty)}=\lim _{s \rightarrow \infty} \rho_{(s)}=\rho_{(0)}-4 \sum_{s=0}^{\infty} \delta_{(s)}=\rho_{(0)}-8 \delta_{(0)}=\frac{\rho_{(0)}}{2},
$$

and that the limit objects are close to the initial (approximate) ones:

$$
\begin{gathered}
\left\|\tau_{(\infty)}-\tau_{(0)}\right\|_{I_{(\infty)}, \rho_{(0)} / 2} \leq \frac{2 \bar{\alpha} \varepsilon_{(0)}}{\gamma_{0}^{2} \delta_{(0)}^{2 \nu}}, \quad\left\|N_{(\infty)}-N_{(0)}\right\|_{I_{(\infty)}, \rho_{(0)} / 2} \leq \frac{2 \bar{\alpha} \varepsilon_{(0)}}{\gamma_{0}^{4} \delta_{(0)}^{4 \nu-1}}, \\
\left|\lambda_{i,(\infty)}-\lambda_{i,(0)}\right|_{I_{(\infty)}} \leq \frac{2 \bar{\alpha} \varepsilon_{(0)}}{\gamma_{0}^{2} \delta_{(0)}^{2 \nu-1}}
\end{gathered}
$$

for $i=1, \ldots, n-r$. Then, from these expressions we obtain bounds (10) and (11) in the statement of the theorem, just observing that $\varepsilon_{(0)} \leq \varepsilon_{*} / \delta_{(0)}$.

\subsection{Lipschitz regularity}

As we pointed out in Section 4.1, to control the measure of the set of removed parameters we cannot use any kind of smooth dependence with respect to $\mu$, because the sets $I_{(s)}$ have empty 
interior. Then, following closely $[33,34,35,36]$, to control this measure we use a Lipschitz condition from below with respect to $\mu$ on the eigenvalues of the matrix $\Lambda_{(s)}$, for $s \geq 0$. In order to guarantee this condition we prove that $\Lambda_{(s)}$ is Lipschitz and then, using that $\Lambda_{(s)}$ is close to $\Lambda_{(0)}$, we can ensure a posteriori that $\Lambda_{(s)}$ is Lipschitz from below. For the sake of completeness, we provide some basic results related to Lipschitz dependence.

Lemma 6.1. Given Lipschitz functions $f, g: I \subset \mathbb{R} \rightarrow \mathbb{C}$, we have

(i) $\operatorname{Lip}_{I}(f+g) \leq \operatorname{Lip}_{I}(f)+\operatorname{Lip}_{I}(g)$.

(ii) $\operatorname{Lip}_{I}(f g) \leq \operatorname{Lip}_{I}(f)\|g\|_{I}+\|f\|_{I} \operatorname{Lip}_{I}(g)$.

(iii) $\operatorname{Lip}_{I}(1 / f) \leq\|1 / f\|_{I}^{2} \operatorname{Lip}_{I}(f)$, if $f$ does not vanish in $I$.

Moreover, an equivalent result holds if $f$ and $g$ take values in spaces of complex matrices ( $f$ must be invertible in the third item) and also for families $\mu \mapsto f_{\mu}$ of functions on $\mathbb{T}^{r}$, using $\operatorname{Lip}_{I, \rho}(f)$ and $\|f\|_{I, \rho}$.

Proof. The result is straightforward.

Lemma 6.2. Given a family $\mu \in I \subset \mathbb{R} \mapsto f_{\mu}$, where $f_{\mu}: \mathcal{U} \subset \mathbb{C}^{l} \rightarrow \mathbb{C}$ is an analytic function with bounded derivatives (that we denote $D^{m} f_{\mu}$ ) in $\mathcal{U}$, and given families $\mu: I \subset \mathbb{R} \mapsto g_{\mu}, h_{\mu}$, where $g_{\mu}, h_{\mu}: \mathbb{T}^{r} \rightarrow \mathcal{U}$ are analytic in $\Delta(\rho)$, we have

(i) $\operatorname{Lip}_{I, \rho}(f \circ g) \leq \operatorname{Lip}_{I, \mathcal{U}}(f)+\|f\|_{I, \mathcal{C}^{1}, \mathcal{U}} \operatorname{Lip}_{I, \rho}(g)$.

(ii) $\operatorname{Lip}_{I, \rho}(f \circ g-f \circ h) \leq \beta^{(1)}\left(\operatorname{Lip}_{I, \rho}(g-h)+\|g-h\|_{I, \rho}\right)$.

(iii) $\operatorname{Lip}_{I, \rho}(f \circ g-f \circ h-D f \circ h[g-h]) \leq \beta^{(2)}\|g-h\|_{I, \rho}\left(\|g-h\|_{I, \rho}+\operatorname{Lip}_{I, \rho}(g-h)\right)$.

The constant $\beta^{(1)}$ depends on $\operatorname{Lip}_{I, \mathcal{C}^{1}, \mathcal{U}}(f),\|f\|_{I, \mathcal{C}^{2}, \mathcal{U}}$ and $\sup _{s \in[0,1]} \operatorname{Lip}_{I, \rho}(h+s(g-h))$. The constant $\beta^{(2)}$ depends on $\operatorname{Lip}_{I, \mathcal{C}^{2}, \mathcal{U}}(f),\|f\|_{I, \mathcal{C}^{3}, \mathcal{U}}$ and $\sup _{s \in[0,1]} \operatorname{Lip}_{I, \rho}(h+s(g-h))$.

Proof. Item (i) is straightforward. Then, items (ii) and (iii) are obtained by using the expressions

$$
f_{\mu} \circ g_{\mu}-f_{\mu} \circ h_{\mu}=\int_{0}^{1} D f_{\mu} \circ\left(h_{\mu}+s\left(g_{\mu}-h_{\mu}\right)\right)\left[g_{\mu}-h_{\mu}\right] d s,
$$

and

$$
f_{\mu} \circ g_{\mu}-f_{\mu} \circ h_{\mu}-D f_{\mu} \circ h_{\mu}\left[g_{\mu}-h_{\mu}\right]=\int_{0}^{1} D^{2} f_{\mu} \circ\left(h_{\mu}+s\left(g_{\mu}-h_{\mu}\right)\right)\left[g_{\mu}-h_{\mu}\right]^{\otimes 2} d s,
$$

respectively, and then applying item (i). 
Lemma 6.3. Let $\mu \in I \subset \mathbb{R} \mapsto g_{\mu}$ be a family of functions $g_{\mu}: \mathbb{T}^{r} \rightarrow \mathbb{C}$ that are analytic in $\Delta(\rho)$ and satisfying $\operatorname{Lip}_{I, \rho}(g)<\infty$. If we expand $g$ in Fourier series

$$
g_{\mu}(\theta)=\sum_{k \in \mathbb{Z}^{r}} \hat{g}_{k}(\mu) \mathrm{e}^{\mathrm{i}\langle k, \theta\rangle}
$$

then we have

(i) $\operatorname{Lip}_{I}\left(\hat{g}_{k}\right) \leq \operatorname{Lip}_{I, \rho}(g) \mathrm{e}^{-|k|_{1} \rho}$.

(ii) $\operatorname{Lip}_{I, \rho-\delta}\left(\frac{\partial g}{\partial \theta_{j}}\right) \leq \frac{1}{\delta} \operatorname{Lip}_{I, \rho}(g)$, for $j=1, \ldots, r$.

(iii) Given $\omega \in \mathbb{R}^{r} \backslash\{0\}$ and a Lipschitz function $d: I \subset \mathbb{R} \rightarrow \mathbb{C}$, we consider the sets $\left\{d_{k}^{0}\right\}_{k \in \mathbb{Z}^{r} \backslash\{0\}},\left\{d_{k}^{1}\right\}_{k \in \mathbb{Z}^{r}}$ of complex functions of $\mu$ given by $d_{k}^{0}=\langle k, \omega\rangle, d_{k}^{1}=\langle k, \omega\rangle+$ $d(\mu)$, satisfying $\left|d_{k}^{0}\right|,\left|d_{k}^{1}\right| \geq \gamma /|k|_{1}^{\nu}$, if $|k|_{1} \neq 0$, for certain $1>\gamma>0$ and $\nu>r-1$. Then, the functions $f^{0}$ and $f^{1}$ whose Fourier coefficients are given by

$$
\begin{array}{rlrl}
\hat{f}_{k}^{0} & =\hat{g}_{k} / d_{k}^{0}, & k \in \mathbb{Z}^{r} \backslash\{0\}, & \hat{f}_{0}^{0}=0, \\
\hat{f}_{k}^{1}=\hat{g}_{k} / d_{k}^{1}, & k \in \mathbb{Z}^{r}, &
\end{array}
$$

satisfy

$$
\begin{aligned}
& \operatorname{Lip}_{I, \rho-\delta}\left(f^{0}\right) \leq \frac{\alpha_{0}}{\gamma \delta^{\nu}} \operatorname{Lip}_{I, \rho-\delta}(g) \\
& \operatorname{Lip}_{I, \rho-\delta}\left(f^{1}\right) \leq \beta_{0}\left(\frac{\operatorname{Lip}_{I, \rho}(g)}{\gamma \delta^{\nu}}+\|g\|_{I, \rho} \frac{\operatorname{Lip}_{I}(d)}{\gamma^{2} \delta^{2 \nu}}\right)+\operatorname{Lip}_{I, \rho}(g)\left\|\frac{1}{d}\right\|_{I}+\left\|\frac{1}{d}\right\|_{I}^{2} \operatorname{Lip}_{I}(d)\|g\|_{I, \rho},
\end{aligned}
$$

for any $\delta \in(0, \min \{1, \rho\})$, where $\alpha_{0} \geq 1$ is the constant that appears in Lemma 5.2, and $\beta_{0} \geq \alpha_{0}$ is a constant depending on $r, \nu$ and $\alpha_{0}$.

Proof. Items (i) and (ii) are straightforward (see [34]). Item (iii) follows from the same arguments used in Lemma 5.2 and applying the properties in Lemma 6.1.

Now, we use these elementary results to control recursively the Lipschitz dependence of the constructed objects. To this end, we obtain an "extended" version of Proposition 5.1.

Lemma 6.4 (Addenda to Proposition 5.1). Let us consider a Lipschitz family of Hamiltonian systems $\mu \in I \subset \mathbb{R} \mapsto h_{\mu}$, where $I$ is an arbitrary set, with $h_{\mu}: U \subset \mathbb{R}^{2 n} \rightarrow \mathbb{R}$, and a vector of basic frequencies $\omega \in \mathbb{R}^{r}$. Assume that there exist families $\mu \in I \mapsto \tau_{\mu}, N_{\mu}, \Lambda_{\mu}$ satisfying all the hypotheses of Proposition 5.1 for every $\mu \in I$ and also that

$$
\|h\|_{I, \mathcal{C}^{4}, \mathcal{U}} \leq \sigma_{0}, \quad \operatorname{Lip}_{I, \mathcal{C}^{3}, \mathcal{U}}(h), \operatorname{Lip}_{I, \rho}(\tau), \operatorname{Lip}_{I, \rho}(D \tau), \operatorname{Lip}_{I, \rho}(N), \operatorname{Lip}_{I}(\Lambda)<\sigma_{5}
$$


Then, there exists a constant $\bar{\beta} \geq \bar{\alpha}$-where $\bar{\alpha}$ is introduced in Proposition 5.1-depending on $r, n, \nu,|\omega|, \sigma_{0}, \sigma_{1}, \sigma_{2}, \sigma_{3}, \sigma_{4}$ and $\sigma_{5}$, such that if the condition

$$
\max \left\{\operatorname{Lip}_{I, \rho}(\tau), \operatorname{Lip}_{I, \rho}(D \tau), \operatorname{Lip}_{I, \rho}(N), \operatorname{Lip}_{I}(\Lambda)\right\}+\frac{\bar{\beta} \hat{\varepsilon}}{\gamma^{4} \delta^{4 \nu-1}}<\sigma_{5}
$$

holds, where

$$
\hat{\varepsilon}=\frac{\operatorname{Lip}_{I, \rho}(e)}{\delta}+\operatorname{Lip}_{I, \rho}(R)+\frac{1}{\gamma \delta^{\nu}}\left(\frac{\|e\|_{I, \rho}}{\delta}+\|R\|_{I, \rho}\right),
$$

then we have that the families $\mu \mapsto \bar{\tau}_{\mu}, D \bar{\tau}_{\mu}, \bar{N}_{\mu}, \bar{\Lambda}_{\mu}, \Delta_{\tau}, \Delta_{N}, \Delta_{\Lambda}$ obtained in Proposition 5.1 satisfy

$$
\begin{gathered}
\operatorname{Lip}_{I, \rho-2 \delta}(\bar{\tau}), \operatorname{Lip}_{I, \rho-3 \delta}(D \bar{\tau}), \operatorname{Lip}_{I, \rho-4 \delta}(\bar{N}), \operatorname{Lip}_{I}(\bar{\Lambda})<\sigma_{5} \\
\operatorname{Lip}_{I, \rho-2 \delta}\left(\Delta_{\tau}\right) \leq \frac{\bar{\beta} \hat{\varepsilon}}{\gamma^{2} \delta^{2 \nu-1}}, \quad \operatorname{Lip}_{I, \rho-4 \delta}\left(\Delta_{N}\right) \leq \frac{\bar{\beta} \hat{\varepsilon}}{\gamma^{4} \delta^{4 \nu-1}}, \quad \operatorname{Lip}_{I}\left(\Delta_{\Lambda}\right) \leq \frac{\bar{\beta} \hat{\varepsilon}}{\gamma^{2} \delta^{2 \nu-1}},
\end{gathered}
$$

and

$$
\operatorname{Lip}_{I, \rho-3 \delta}(\bar{e}) \leq \frac{\bar{\beta} \hat{\varepsilon}^{2}}{\gamma^{3} \delta^{3 \nu-2}}, \quad \operatorname{Lip}_{I, \rho-4 \delta}(\bar{R}) \leq \frac{\bar{\beta} \hat{\varepsilon}^{2}}{\gamma^{7} \delta^{7 \nu-2}}
$$

Proof. Basically, it consists in using the properties in Lemmata 6.1, 6.2 and 6.3 to control the different functions that appear along the proof of Propositions 5.3 (construction of the approximately symplectic basis) and Proposition 5.1 (iterative procedure). Since the computations are similar as those detailed in Section 5, we will omit some intermediate steps.

First, let us study the objects in Proposition 5.3. To this end, we observe that there exists a constant $\hat{\beta}$ (which is enlarged along the proof in order to include dependence on $r, n, \nu,|\omega|, \sigma_{0}$, $\sigma_{1}, \sigma_{2}, \sigma_{3}, \sigma_{4}$ and $\left.\sigma_{5}\right)$ such that

$$
\begin{gathered}
\operatorname{Lip}_{I, \rho}\left(G_{D \tau}^{-1}\right), \operatorname{Lip}_{I, \rho}\left(G_{N, N^{*}}^{-1}\right), \operatorname{Lip}_{I, \rho}\left(T_{i}\right), \operatorname{Lip}_{I}\left(\left[A_{1}\right]_{\mathbb{T}^{r}}^{-1}\right), \operatorname{Lip}_{I, \rho}(V), \operatorname{Lip}_{I, \rho}\left(B_{j}\right) \leq \hat{\beta}, \\
\operatorname{Lip}_{I, \rho-\delta}\left(L_{\omega} B_{i}\right) \leq \frac{\hat{\beta}}{\delta}
\end{gathered}
$$

for $i=1,2$ and $j=1,2,3$. For example, we have that

$$
\begin{aligned}
\operatorname{Lip}_{I, \rho}\left(G_{D \tau}^{-1}\right) & \leq\left\|G_{D \tau}^{-1}\right\|_{I, \rho}^{2} \operatorname{Lip}_{I, \rho}\left(G_{D \tau}\right) \\
& \leq\left\|G_{D \tau}^{-1}\right\|_{I, \rho}^{2}\left(\operatorname{Lip}_{I, \rho}\left(D \tau^{\top}\right)\|D \tau\|_{I, \rho}+\left\|D \tau^{\top}\right\|_{I, \rho} \operatorname{Lip}_{I, \rho}(D \tau)\right) \\
& \leq 4 n\left\|G_{D \tau}^{-1}\right\|_{I, \rho}^{2}\|D \tau\|_{I, \rho} \operatorname{Lip}_{I, \rho}(D \tau) \leq \hat{\beta}
\end{aligned}
$$

Then, we estimate Lipschitz constants for the matrices $\Omega_{D \tau}, \Omega_{N_{1}}, \ldots, \Omega_{V}$ that characterize the approximately symplectic character of the basis in Propositions 5.3. For example, we get 
$\operatorname{Lip}_{I, \rho-2 \delta}\left(\Omega_{D \tau}\right)$ by applying item (iii) of Lemma 6.3 to the $(i, j)$-component of $\Omega_{D \tau}$ obtained from equation (80), i.e., taking $d_{k}^{0}=\langle\omega, k\rangle$ and $g=-\mathrm{i}\left(\Omega_{D e, D \tau}+\Omega_{D \tau, D e}\right)^{(i, j)}$, thus obtaining

$$
\operatorname{Lip}_{I, \rho-2 \delta}\left(\Omega_{D \tau}\right) \leq \frac{\hat{\beta}}{\gamma \delta^{\nu+1}}\left(\operatorname{Lip}_{I, \rho}(e)+\|e\|_{I, \rho}\right) .
$$

Similarly, to bound $\operatorname{Lip}_{I, \rho-\delta}\left(\Omega_{N_{1}}\right)$ we proceed in the same way taking $d_{k}^{1}=\langle k, \omega\rangle+\lambda_{i}+\lambda_{j}$ and $g=-\mathrm{i}\left(\Omega_{R, N_{1}}+\Omega_{N_{1}, R}\right)^{(i, j)}$. We obtain

$$
\operatorname{Lip}_{I, \rho-\delta}\left(\Omega_{N_{1}}\right) \leq \frac{\hat{\beta}}{\gamma \delta^{\nu}}\left(\operatorname{Lip}_{I, \rho}(R)+\frac{\|R\|_{I, \rho}}{\gamma \delta^{\nu}}\right) .
$$

In this way, we have the following bounds in terms of the error $\hat{\varepsilon}$ defined in (110)

$$
\begin{array}{r}
\operatorname{Lip}_{I, \rho-2 \delta}\left(\Omega_{D \tau}\right), \operatorname{Lip}_{I, \rho-\delta}\left(\Omega_{N_{i}}\right), \operatorname{Lip}_{I, \rho-\delta}\left(\Omega_{N_{2}, N_{1}}\right) \leq \frac{\hat{\beta} \hat{\varepsilon}}{\gamma \delta^{\nu}}, \\
\operatorname{Lip}_{I, \rho-2 \delta}\left(\Omega_{D \tau, N_{i}}\right), \operatorname{Lip}_{I, \rho-2 \delta}\left(\Omega_{V, D \tau}\right), \operatorname{Lip}_{I, \rho-2 \delta}\left(\Omega_{V, N_{i}}\right), \operatorname{Lip}_{I, \rho-2 \delta}\left(\Omega_{V}\right) \leq \frac{\hat{\beta} \hat{\varepsilon}}{\gamma \delta^{\nu}},
\end{array}
$$

for $i=1,2$. Furthermore, by performing similar computations to estimate the Lipschitz constants of $M_{3}$ in (86), $C_{1}^{+}$in (89), $C_{2}^{+}$in (88), $C_{3}^{+}$in (87) and $\left(\mathrm{Id}+M_{3}\right)^{-1}$ in (92) we obtain that the functions $A_{i}^{+}$, for $i=1, \ldots, 4$, in the statement of Proposition 5.3 are controlled by

$$
\operatorname{Lip}_{I, \rho-2 \delta}\left(A_{i}^{+}\right) \leq \frac{\hat{\beta} \hat{\varepsilon}}{\gamma \delta^{\nu+1}},
$$

provided $\hat{\varepsilon}$ is small enough —indeed, under condition (51) in Proposition 5.1.

Now we can estimate the Lipschitz constant of $\Delta_{i}, i=1, \ldots, 4$, defined as the solutions of cohomological equations (37)-(40). In analogy with the notation in Proposition 5.1, we introduce a constant $\bar{\beta} \geq \hat{\beta}$ depending on the same variables as $\hat{\beta}$. We have

$$
\operatorname{Lip}_{I, \rho-2 \delta}\left(\Delta_{1}\right) \leq \frac{\bar{\beta} \hat{\varepsilon}}{\gamma^{2} \delta^{2 \nu-1}}, \quad \operatorname{Lip}_{I, \rho-\delta}\left(\Delta_{i}\right) \leq \frac{\bar{\beta} \hat{\varepsilon}}{\gamma \delta^{\nu-1}}, \quad \operatorname{Lip}_{I, \rho-2 \delta}\left(\Delta_{\tau}\right) \leq \frac{\bar{\beta} \hat{\varepsilon}}{\gamma^{2} \delta^{2 \nu-1}},
$$

for $i=2,3,4$. In particular, we observe that condition (109) guarantees that

$$
\operatorname{Lip}_{I, \rho-2 \delta}(\bar{\tau}), \operatorname{Lip}_{I, \rho-3 \delta}(D \bar{\tau})<\sigma_{5}
$$

Now, to control the Lipschitz constant of expression (94) we compute

$$
\left.\operatorname{Lip}_{I, \rho-2 \delta}\left(D_{i}\right), \operatorname{Lip}_{I, \rho-2 \delta}\left(e^{+}\right) \leq \bar{\beta} \delta \hat{\varepsilon}^{2}, \quad \operatorname{Lip}_{I, \rho-3 \delta}\left(\mathcal{R}\left(\Delta_{\tau}\right)+e\right)\right) \leq \frac{\bar{\beta} \hat{\varepsilon}^{2}}{\gamma \delta^{\nu}}
$$


for $i=1,2,3,4$. Finally, an estimate for the Lipschitz constant of $\bar{e}$ follows by applying item (iii) in Lemma 6.2 that allows controlling the Taylor remainder, thus obtaining

$$
\operatorname{Lip}_{I, \rho-3 \delta}(\bar{e}) \leq \frac{\bar{\beta} \hat{\varepsilon}^{2}}{\gamma^{3} \delta^{3 \nu-2}}
$$

Similarly we control the Lipschitz constant of the new normal eigenvalues of the new reducibility error. As in Section 5, we start by controlling

$$
\operatorname{Lip}_{I, \rho-2 \delta}(\hat{R}) \leq \frac{\bar{\beta} \hat{\varepsilon}}{\gamma^{2} \delta^{2 \nu-1}}, \quad \operatorname{Lip}_{I}\left(\Delta_{\Lambda}\right) \leq \frac{\bar{\beta} \hat{\varepsilon}}{\gamma^{2} \delta^{2 \nu-1}}
$$

where we used item (ii) in Lemma 6.2, and then we apply Lemma 6.3 in order to obtain

$$
\operatorname{Lip}_{I, \rho-3 \delta}\left(P_{i}\right) \leq \frac{\bar{\beta} \hat{\varepsilon}}{\gamma^{3} \delta^{3 \delta-1}}, \quad \operatorname{Lip}_{I, \rho-4 \delta}\left(P_{1}\right) \leq \frac{\bar{\beta} \hat{\varepsilon}}{\gamma^{4} \delta^{4 \delta-1}},
$$

for $i=2,3,4$. From these estimates it follows that

$$
\operatorname{Lip}_{I, \rho-4 \delta}\left(\hat{\Delta}_{N}\right), \operatorname{Lip}_{I}\left(d_{i}\right), \operatorname{Lip}_{I}\left(b_{i}\right), \operatorname{Lip}_{I, \rho-4 \delta}\left(\Delta_{N}\right) \leq \frac{\bar{\beta} \hat{\varepsilon}}{\gamma^{4} \delta^{4 \nu-1}},
$$

and we observe that condition (109) guarantees that

$$
\operatorname{Lip}_{I, \rho-4 \delta}(\bar{N}), \operatorname{Lip}_{I}(\bar{\Lambda})<\sigma_{5} .
$$

In order to control the Lipschitz constant of the new error in reducibility $\bar{R}$ we have to compute

$$
\operatorname{Lip}_{I, \rho-2 \delta}\left(R^{+}\right) \leq \frac{\bar{\beta} \hat{\varepsilon}^{2}}{\gamma^{2} \delta^{2 \nu-1}}, \quad \operatorname{Lip}_{I, \rho-4 \delta}\left(\mathcal{S}\left(\hat{\Delta}_{N}, \Delta_{\Lambda}\right)+\hat{R}\right) \leq \frac{\bar{\beta} \hat{\varepsilon}^{2}}{\gamma^{3} \delta^{3 \nu}}
$$

and

$$
\operatorname{Lip}_{I, \rho-4 \delta}\left(\mathcal{S}\left(\Delta_{N}, \Delta_{\Lambda}\right)+\hat{R}\right) \leq \frac{\bar{\beta} \hat{\varepsilon}^{2}}{\gamma^{7} \delta^{7 \nu-2}}
$$

Finally, estimate (113) for the Lipschitz constant of $\bar{R}$ follows by applying Lemma 6.2 that allows us to control the Taylor remainder in (98).

In order to prove that the Lipschitz dependence is preserved along the iterative scheme, we only have to check - together with conditions for the convergence of the quadratic methodthat condition (109) is satisfied at every step of the procedure. As in Section 6.1, assuming that we have applied $s$ times Proposition 5.1 and Lemma 6.4, we have to ensure that $\max \left\{\operatorname{Lip}_{I_{(s-1)}, \rho_{(s)}}\left(\tau_{(s)}\right), \operatorname{Lip}_{I_{(s-1)}, \rho_{(s)}}\left(D \tau_{(s)}\right), \operatorname{Lip}_{I_{(s-1)}, \rho_{(s)}}\left(N_{(s)}\right), \operatorname{Lip}_{I_{(s-1)}}\left(\Lambda_{(s)}\right)\right\}+\frac{\bar{\beta} \hat{\varepsilon}_{(s)}}{\gamma_{(s)}^{4} \delta_{(s)}^{4 \nu-1}}<\sigma_{5}$ 
To this end, we compute the normalized error (110) at the $s$-th step

$$
\hat{\varepsilon}_{(s)}=\frac{\operatorname{Lip}_{I_{(s-1)}, \rho_{(s)}}\left(e_{(s)}\right)}{\delta_{(s)}}+\operatorname{Lip}_{I_{(s-1)}, \rho_{(s)}}\left(R_{(s)}\right)+\frac{1}{\gamma_{(s)} \delta_{(s)}^{\nu}}\left(\frac{\left\|e_{(s)}\right\|_{I_{(s-1)}, \rho_{(s)}}}{\delta_{(s)}}+\left\|R_{(s)}\right\|_{I_{(s-1)}, \rho_{(s)}}\right)
$$

in terms of $\hat{\varepsilon}_{(0)}$. Analogous computations as those performed in (106) show that

$$
\hat{\varepsilon}_{(s)} \leq \frac{2^{(s-1)(7 \nu-2)+\nu+2} \bar{\beta}}{\gamma_{0}^{7} \delta_{(0)}^{7 \nu-2}} \hat{\varepsilon}_{(s-1)}^{2}
$$

where we used (57), (60) and (113). Then, by iterating this sequence backwards, we obtain that

$$
\hat{\varepsilon}_{(s)} \leq \frac{\gamma_{0}^{7} \delta_{(0)}^{7 \nu-2}}{\bar{\beta}} 2^{-(s+1)(7 \nu-2)-\nu-2}\left(\frac{2^{8 \nu} \bar{\beta} \hat{\varepsilon}_{(0)}}{\gamma_{0}^{7} \delta_{(0)}^{7 \nu-2}}\right)^{2^{s}}
$$

and the convergence of the Lipschitz procedure follows from similar computations as those in (107) (but using (112)), asking for the condition

$$
\frac{2^{8 \nu} \bar{\beta} \hat{\varepsilon}_{(0)}}{\gamma_{0}^{7} \delta_{(0)}^{7 \nu-2}} \leq \frac{1}{2} \min \left\{1, \sigma_{5}-\sigma^{* * *}\right\}
$$

where

$$
\sigma^{* * *}=\max \left\{\operatorname{Lip}_{I, \rho_{(0)}}\left(\tau_{(0)}\right), \operatorname{Lip}_{I, \rho_{(0)}}\left(D \tau_{(0)}\right), \operatorname{Lip}_{I, \rho_{(0)}}\left(N_{(0)}\right), \operatorname{Lip}_{I}\left(\Lambda_{(0)}\right)\right\} .
$$

Next, we show that the Lipschitz constants from below of the functions

$$
\mu \in I_{(s-1)} \mapsto \lambda_{i,(s)}(\mu), \quad \mu \in I_{(s-1)} \mapsto \lambda_{i,(s)}(\mu) \pm \lambda_{j,(s)}(\mu)
$$

for $i \neq j=1, \ldots, n-r$, have a lower bound that does not depend on the step $s$-notice that Lipschitz (from above) constants are controlled for every $s$ as (111). Indeed, we have

$$
\operatorname{Lip}_{I_{(s-1)}}\left(\Lambda_{(s)}-\Lambda_{(0)}\right) \leq \sum_{j=0}^{s-1} \operatorname{Lip}_{I_{(j)}}\left(\Delta_{\Lambda_{(j)}}\right) \leq \frac{2 \bar{\beta} \hat{\varepsilon}_{(0)}}{\gamma_{0}^{2} \delta_{(0)}^{2 \nu-1}}
$$

where we used condition (115).

Finally, using that

$$
\operatorname{lip}_{I_{(s-1)}}\left(\lambda_{i,(s)}\right) \geq \operatorname{lip}_{I}\left(\lambda_{i,(0)}\right)-\operatorname{Lip}_{I_{(s-1)}}\left(\lambda_{i,(s)}-\lambda_{i,(0)}\right)
$$

(and analogous computations hold for $\lambda_{i,(s)}(\mu) \pm \lambda_{j,(s)}(\mu)$ ) we end up with the bounds

$$
\operatorname{lip}_{I_{(s-1)}}\left(\lambda_{i,(s)}\right) \geq \frac{\sigma_{6}}{4}, \quad \operatorname{lip}_{I_{(s-1)}}\left(\lambda_{i,(s)} \pm \lambda_{j,(s)}\right) \geq \frac{\sigma_{6}}{2},
$$


for $i, j=1, \ldots, n-r$, with $i \neq j$, and $s \geq 0$, provided that -we use $\mathrm{H}_{6}$ in Theorem 3.1-

$$
\frac{2 \bar{\beta} \hat{\varepsilon}_{(0)}}{\gamma_{0}^{2} \delta_{(0)}^{2 \nu-1}}<\frac{\sigma_{6}}{4} .
$$

Therefore, the quadratic procedure to obtain invariant tori with Lipschitz dependence converges for $\mu \in I_{(\infty)}$ —see (108)— provided conditions (100), (115) and (117) hold. Moreover, notice that if we control the errors $\varepsilon_{(0)}$ and $\hat{\varepsilon}_{(0)}$ (given by (99) and (114), respectively) in terms of the error $\varepsilon_{*}$ that appears in the statement of Theorem 3.1 as follows

$$
\varepsilon_{(0)} \leq \frac{\varepsilon_{*}}{\delta_{(0)}}, \quad \hat{\varepsilon}_{(0)} \leq \frac{\varepsilon_{*}}{\gamma_{0} \delta_{(0)}^{\nu+1}},
$$

then we have convergence provided that $\varepsilon_{*} \leq C_{1} \gamma_{0}^{8}$, where $C_{1}$ is taken in order to meet all the required conditions.

\subsection{Measure of the set of excluded parameters}

It remains to control the measure of the set $I_{(\infty)}$ given by (103) and (108), for which all steps performed along the iterative procedure of Section 6.1 are well-posed. Let us recall that $I_{(\infty)}$ is constructed by taking out, in recursive form, the set of parameters $\mu$ for which (4) and (9) do not hold at any step of the KAM process. Concretely, we bound the measure of the complementary set $I \backslash I_{(\infty)}$, that we write as

$$
I \backslash I_{(\infty)}=\left(I \backslash I_{(0)}\right) \cup\left(I_{(0)} \backslash I_{(\infty)}\right)=\left(I \backslash I_{(0)}\right) \cup \bigcup_{s \geq 1} I_{(s-1)} \backslash I_{(s)} .
$$

We start by controlling the measure of $I_{(s-1)} \backslash I_{(s)}$, for $s \geq 1$. To simplify the notation, in the following discussion we consider a generic divisor of the form $\langle\omega, k\rangle-d_{(s)}(\mu)$, where $d_{(s)}(\mu)$ is either $\lambda_{j,(s)}(\mu)$ or $\lambda_{j,(s)}(\mu) \pm \lambda_{i,(s)}(\mu)$. For this purpose, we introduce the $k$-th resonant set for the divisor $d_{(s)}$ as

$$
\operatorname{Res}_{k}^{(s)}=\left\{\mu \in I_{(s-1)}:\left|\langle\omega, k\rangle-d_{(s)}(\mu)\right|<\frac{\gamma_{(s)}}{|k|_{1}^{\nu}}\right\},
$$

and we control

$$
\operatorname{meas}_{\mathbb{R}}\left(\bigcup_{k \in \mathbb{Z}^{r} \backslash\{0\}} \operatorname{Res}_{k}^{(s)}\right)
$$

using the following two elementary results.

Lemma 6.5. In the above setting, let us assume that $\mu \in I_{(s-1)}$, with $s \geq 1$. Then, there exists $K^{*}(s) \in \mathbb{N}$ such that $\mu \notin \operatorname{Res}_{k}^{(s)}$ provided $|k|_{1} \leq K^{*}(s)$. Concretely,

$$
K^{*}(s)=\left\lfloor\tilde{\varepsilon}^{-\frac{2^{s-1}}{\nu}}\right\rfloor, \quad \text { where } \quad \tilde{\varepsilon}=\frac{2^{8 \nu-1} \bar{\alpha} \varepsilon_{(0)}}{\gamma_{0}^{8} \delta_{(0)}^{8 \nu-2}} .
$$


Proof. To prove this result, we observe that the correction of the normal frequencies - and hence of of $d_{(s)}(\mu)$ - is smaller at each step of the iterative procedure. Indeed, using (58) at the $s$-th step, (105), (106) and (118), we have that

$$
\left\|d_{(s)}-d_{(s-1)}\right\|_{I_{(s-1)}} \leq 2\left\|\Delta_{\Lambda_{(s-1)}}\right\|_{I_{(s-1)}} \leq \gamma_{0}^{6} \delta_{(0)}^{6 \nu-1} 2^{-(6 \nu-1) s-2 \nu+1} \tilde{\varepsilon}^{2^{s-1}} .
$$

Then, given $\mu \in I_{(s-1)}$, it turns out that $\mu \notin \operatorname{Res}_{k}^{(s)}$ provided the quantity

$$
\begin{aligned}
\left|\langle\omega, k\rangle-d_{(s)}(\mu)\right| & \geq\left|\langle k, \omega\rangle-d_{(s-1)}(\mu)\right|-\left\|d_{(s)}-d_{(s-1)}\right\|_{I_{(s-1)}} \\
& \geq \gamma_{(s-1)}|k|_{1}^{-\nu}-\gamma_{0}^{6} \delta_{(0)}^{6 \nu-1} 2^{-(6 \nu-1) s-2 \nu+1} \tilde{\varepsilon}^{2^{s-1}}
\end{aligned}
$$

is larger than $\gamma_{(s)}|k|_{1}^{-\nu}$. This is equivalent to ask for (recall that $\left.\gamma_{(s)}=\left(1+2^{-s}\right) \gamma_{0}\right)$

$$
\gamma_{0}^{6} \delta_{(0)}^{6 \nu-1} 2^{-(6 \nu-1) s-2 \nu+1} \tilde{\varepsilon}^{2^{s-1}} \leq 2^{-s} \gamma_{0}|k|_{1}^{-\nu},
$$

which is satisfied for every $|k|_{1} \leq K^{*}(s)$, where $K^{*}(s)$ is given in (120).

Lemma 6.6. Let us consider a vector $\omega \in \mathbb{R}^{r}$ satisfying Diophantine conditions (4) of $(\hat{\gamma}, \hat{\nu})$ type, with $\hat{\gamma}>0$ and $\hat{\nu}>r-1$. Then, given $J \subset[\alpha, \beta] \subset \mathbb{R}$, with $\alpha>0, \gamma>0, \nu>\hat{\nu}$ and $K \in \mathbb{N}$, we have that the measure of the set

$$
\Delta_{K}=\left\{d \in J:|\langle k, \omega\rangle-d|<\frac{\gamma}{|k|_{1}^{\nu}}, \text { for some } k \in \mathbb{Z}^{r}, \text { with }|k|_{1}>K\right\}
$$

is controlled as

$$
\operatorname{meas}_{\mathbb{R}}\left(\Delta_{K}\right) \leq 2^{\hat{\nu}+1} \hat{\nu}(\beta-\alpha) \frac{\gamma}{\hat{\gamma}} \sum_{j>K} \frac{1}{j^{\nu-\hat{\nu}+1}} .
$$

Proof. Let us introduce the following notation

$$
\operatorname{Res}_{k}=\left\{d \in J:|\langle k, \omega\rangle-d|<\frac{\gamma}{|k|_{1}^{\nu}}\right\}
$$

if $|k|_{1}>K$, $\operatorname{Res}_{k}=\varnothing$ if $|k|_{1} \leq K$, and also $\widetilde{\operatorname{Res}_{j}}=\bigcup_{|k|_{1}=j} \operatorname{Res}_{k}$. Then, we have that

$$
\operatorname{meas}_{\mathbb{R}}\left(\Delta_{K}\right)=\operatorname{meas}_{\mathbb{R}}\left(\bigcup_{j>K} \widetilde{\operatorname{Res}_{j}}\right) \leq \sum_{j>K} \operatorname{meas}_{\mathbb{R}}\left(\widetilde{\operatorname{Res}_{j}}\right)
$$

thus reducing the problem to study the sets $\widetilde{\operatorname{Res}_{j}}$, which only contain resonances of order $j$. Now, let us observe that the width of one resonant set $\operatorname{Res}_{k}$ of order $j$ is controlled by $\operatorname{meas}_{\mathbb{R}}\left(\operatorname{Res}_{k}\right) \leq 2 \gamma j^{-\nu}$. Hence, it remains to estimate the number of resonant sets Res $k$, with $|k|_{1}=j$, that intersect $J$. This follows using similar arguments as in Lemma 5.2. 
For this purpose we introduce some notation. We define $d_{k}=\langle k, \omega\rangle$, which corresponds to the exact resonant value $d \in \operatorname{Res}_{k}$, and

$$
\tilde{D}_{j}=[\alpha, \beta] \cap\left(\bigcup_{|k|_{1}=j}\left\{d_{k}\right\}\right), \quad \hat{D}_{j}=\bigcup_{l \leq j} \tilde{D}_{l} .
$$

With these ingredients, we have

$$
\operatorname{meas}_{\mathbb{R}}\left(\Delta_{K}\right) \leq \sum_{j>K} \#\left(\tilde{D}_{j}\right) \frac{2 \gamma}{j^{\nu}}=2 \gamma \sum_{j>K} \# \hat{D}_{j}\left(\frac{1}{j^{\nu}}-\frac{1}{(j+1)^{\nu}}\right) \leq 2 \gamma \nu \sum_{j>K} \frac{\# \hat{D}_{j}}{j^{\nu+1}}
$$

where we used the convention $\operatorname{Res}_{k}=\varnothing$ if $|k|_{1} \leq K$.

In order to estimate $\# \hat{D}_{j}$, we sort the resonances $d_{k}$ for $|k|_{1} \leq j$ according to

$$
\cdots<d_{k_{-2}}<d_{k_{-1}}<0<d_{k_{1}}<d_{k_{2}}<\cdots
$$

and we observe that $d_{k_{l}} \neq d_{k_{j}}$ if $k_{l} \neq k_{j}$. Then, using that $\omega$ is Diophantine of $(\hat{\gamma}, \hat{\nu})$-type, we have $\left|d_{k_{j+1}}-d_{k_{j}}\right| \geq \hat{\gamma} /(2 j)^{\hat{\nu}}$. Hence:

$$
\#\left(\hat{D}_{j}\right) \leq \frac{|\beta-\alpha| 2^{\hat{\nu}} j^{\hat{\nu}}}{\hat{\gamma}}
$$

By introducing this expression into (123) we end up with (122).

Now let us control (119). On the one hand, we use Lemma 6.5 in order restrict the indexes in (119) to $k \in \mathbb{Z}^{r} \backslash\{0\}$ such that $|k|_{1}>K^{*}(s)$ - see (120) - and, on the other hand, we use Lemma 6.6 to control the corresponding measure. To do that, we observe that $\operatorname{lip}_{I_{(s-1)}}\left(d_{(s)}\right) \geq \sigma_{6} / 4$ by (116) and that $d_{(s)}\left(I_{(s-1)}\right) \subset\left[\sigma_{3} / 2, \sigma_{4}\right]$ - this follows from the fact that (64) is preserved along the iterative procedure. Then, the Lipschitz constant from below of $d_{(s)}$ allows moving the measure of the "resonant" sets (121), controlled in terms of $d=d_{(s)}(\mu)$, to the corresponding measure in terms of $\mu$. Hence, we get

$$
\operatorname{meas}_{\mathbb{R}}\left(\bigcup_{k \in \mathbb{Z}^{r} \backslash\{0\}} \operatorname{Res}_{k}^{(s)}\right)=\operatorname{meas}_{\mathbb{R}}\left(\bigcup_{|k|_{1}>K^{*}(s)} \operatorname{Res}_{k}^{(s)}\right) \leq 2^{\hat{\nu}+4} \hat{\nu}\left(\frac{\sigma_{4}-\frac{\sigma_{3}}{2}}{\sigma_{6}}\right) \frac{\gamma_{0}}{\hat{\gamma}} \sum_{j>K^{*}(s)} \frac{1}{j^{\nu-\hat{\nu}+1}},
$$

where we used that $\gamma_{(s)} \leq 2 \gamma_{0}$. Notice that this estimate does not depend on the selected $d_{(s)}(\mu)$, so we can control the measure of the set $I_{(0)} \backslash I_{(\infty)}$ as follows — we multiply the obtained bound of the measure by $2(n-r)^{2}$ to take into account all possible combinations of normal frequencies-

$$
\operatorname{meas}_{\mathbb{R}}\left(I_{(0)} \backslash I_{(\infty)}\right) \leq \overbrace{\frac{\overbrace{}^{\hat{\nu}+5} \hat{\nu}(n-r)^{2}}{\hat{\gamma}}\left(\frac{\sigma_{4}-\frac{\sigma_{3}}{2}}{\sigma_{6}}\right)}^{C} \gamma_{0} \sum_{s=1}^{\infty} \sum_{j>K^{*}(s)} \frac{1}{j^{\nu-\hat{\nu}+1}} \leq \frac{C \gamma_{0}}{\nu-\hat{\nu}} \sum_{s=1}^{\infty} \frac{1}{K^{*}(s)^{\nu-\hat{\nu}}},
$$


where we used $\nu>\hat{\nu}$. Then, we introduce the expression (120) for $K^{*}(s)$ and using that $\tilde{\varepsilon} \leq 1 / 2($ see $(100))$ and that $s \leq 2^{s-1}$, we have

$$
\operatorname{meas}_{\mathbb{R}}\left(I_{(0)} \backslash I_{(\infty)}\right) \leq \frac{C \gamma_{0}}{\nu-\hat{\nu}} \sum_{s=1}^{\infty}\left(\tilde{\varepsilon}^{\frac{\nu-\hat{\nu}}{\nu}}\right)^{2^{s-1}} \leq \frac{C \gamma_{0}}{\nu-\hat{\nu}} \sum_{s=1}^{\infty} 2^{-s} \leq \frac{C}{\nu-\hat{\nu}} \gamma_{0}
$$

Finally, we estimate the measure of the set $I \backslash I_{(0)}=I_{(-1)} \backslash I_{(0)}$ using the same arguments, and it turns out that it is also proportional to $\gamma_{0}$. Hence, we obtain (12) as follows

$$
\operatorname{meas}_{\mathbb{R}}\left(I \backslash I_{(\infty)}\right) \leq \operatorname{meas}_{\mathbb{R}}\left(I \backslash I_{(0)}\right)+\operatorname{meas}_{\mathbb{R}}\left(I_{(0)} \backslash I_{(\infty)}\right) \leq C_{3} \gamma_{0} .
$$

\section{Acknowledgements}

We wish to thank Rafael de la Llave for valuable comments and suggestions, and also Yannick Sire for encouragement and for interesting discussion on reference [20] before it appeared. The authors have been partially supported by MCyT/FEDER grant MTM2006-00478, MICINNFEDER grant MTM2009-06973 and CUR-DIUE grant 2009SGR859. Moreover, the research of A. L. has been supported by the fellows FPU AP2005-2950 and PTA2008-1693-P.

\section{References}

[1] V.I. Arnol'd. Proof of a theorem of A. N. Kolmogorov on the invariance of quasi-periodic motions under small perturbations. Russian Math. Surveys, 18(5):9-36, 1963.

[2] J.B. Bost. Tores invariants des systèmes dynamiques hamiltoniens (d'après Kolmogorov, Arnold, Moser, Rüssmann, Zehnder, Herman, Pöschel, ...). Astérisque, (133-134):113157, 1986. Seminar Bourbaki, Vol. 1984/85.

[3] J. Bourgain. Construction of quasi-periodic solutions for Hamiltonian perturbations of linear equations and applications to nonlinear PDE. Internat. Math. Res. Notices, (11):475ff., approx. 21 pp. (electronic), 1994.

[4] J. Bourgain. On Melnikov's persistency problem. Math. Res. Lett., 4(4):445-458, 1997.

[5] H.W. Broer, H. Hanßmann, À. Jorba, J. Villanueva, and F. Wagener. Normal-internal resonances in quasi-periodically forced oscillators: a conservative approach. Nonlinearity, 16(5):1751-1791, 2003.

[6] H.W. Broer, G.B. Huitema, and M.B. Sevryuk. Quasi-periodic motions in families of dynamical systems. Lecture Notes in Mathematics. Springer-Verlag, Berlin, 1996. Order amidst chaos. 
[7] H.W. Broer, G.B. Huitema, and F. Takens. Unfoldings and bifurcations of quasi-periodic tori. Mem. Amer. Math. Soc., 83(421):viii+175, 1990.

[8] A.D. Bruno. Normalization of a Hamiltonian system near an invariant cycle or torus. Russian Math. Surveys, 44(2):53-89, 1989.

[9] R. Calleja and R. de la Llave. Fast numerical computation of quasi-periodic equilibrium states in 1-d statistical mechanics. Nonlinearity, 22(1):1311-1336, 2009.

[10] E. Castellà and À. Jorba. On the vertical families of two-dimensional tori near the triangular points of the bicircular problem. Celestial Mech. Dynam. Astronom., 76(1):35-54, 2000 .

[11] A. Celletti and L. Chierchia. On the stability of realistic three-body problems. Comm. Math. Phys., 186(2):413-449, 1997.

[12] R. de la Llave. A tutorial on KAM theory. In Smooth ergodic theory and its applications (Seattle, WA, 1999), volume 69 of Proc. Sympos. Pure Math., pages 175-292. Amer. Math. Soc., 2001.

[13] R. de la Llave, A. González, À. Jorba, and J. Villanueva. KAM theory without action-angle variables. Nonlinearity, 18(2):855-895, 2005.

[14] R. de la Llave, G. Huguet, and Y. Sire. Fast numerical algorithms for the computation of invariant tori in Hamiltonian Systems. Preprint available electronically at http: / / www . ma.utexas.edu/mp_arc-bin/mpa?yn=09-2.

[15] R. de la Llave and C. E. Wayne. Whiskered and low dimensional tori in nearly integrable Hamiltonian systems. Math. Phys. Electron. J., 10:Paper 5, 45 pp. (electronic), 2004.

[16] A. Delshams and P. Gutiérrez. Estimates on invariant tori near an elliptic equilibrium point of a Hamiltonian system. J. Differential Equations, 131(2):277-303, 1996.

[17] L.H. Eliasson. Perturbations of stable invariant tori for Hamiltonian systems. Ann. Scuola Norm. Sup. Pisa Cl. Sci. (4), 15(1):115-147 (1989), 1988.

[18] L.H. Eliasson. Floquet solutions for the 1-dimensional quasi-periodic Schrödinger equation. Comm. Math. Phys., 146(3):447-482, 1992.

[19] L.H. Eliasson. Almost reducibility of linear quasi-periodic systems. In Smooth ergodic theory and its applications (Seattle, WA, 1999), volume 69 of Proc. Sympos. Pure Math., pages 679-705. Amer. Math. Soc., Providence, RI, 2001.

[20] E. Fontich, R. de la Llave, and Y. Sire. Construction of invariant whiskered tori by a parametrization method. Part I: Maps and flows in finite dimensions. J. Differential Equations, 246:3136-3213, 2009. 
[21] G. Gómez, À. Jorba, C. Simó, and J. Masdemont. Dynamics and mission design near libration points. Vol. III, volume 4 of World Scientific Monograph Series in Mathematics. World Scientific Publishing Co. Inc., River Edge, NJ, 2001. Advanced methods for triangular points.

[22] G. Gómez, À. Jorba, C. Simó, and J. Masdemont. Dynamics and mission design near libration points. Vol. IV, volume 5 of World Scientific Monograph Series in Mathematics. World Scientific Publishing Co. Inc., River Edge, NJ, 2001. Advanced methods for triangular points.

[23] S.M. Graff. On the conservation of hyperbolic invariant tori for Hamiltonian systems. $J$. Differential Equations, 15:1-69, 1974.

[24] Y. Han, Y. Li, and Y. Yi. Degenerate lower-dimensional tori in Hamiltonian systems. J. Differential Equations, 227(2):670-691, 2006.

[25] A. Haro and R. de la Llave. A parameterization method for the computation of invariant tori and their whiskers in quasi-periodic maps: explorations and mechanisms for the breakdown of hyperbolicity. SIAM J. Appl. Dyn. Syst., 6(1):142-207 (electronic), 2007.

[26] H.-L. Her and J You. Full measure reducibility for generic one-parameter family of quasiperiodic linear systems. J. Dynam. Differential Equations, 20(4):831-866, 2008.

[27] M.R. Herman. Sur les courbes invariantes par les difféomorphismes de l'anneau. Vol. 1, volume 103 of Astérisque. Société Mathématique de France, Paris, 1983. With an appendix by Albert Fathi, With an English summary.

[28] D. Huang and Z. Liu. On the persistence of lower-dimensional invariant hyperbolic tori for smooth Hamiltonian systems. Nonlinearity, 13(1):189-202, 2000.

[29] R. Johnson and J. Moser. The rotation number for almost periodic potentials. Comm. Math. Phys., 84(3):403-438, 1982.

[30] R.A. Johnson and G.R. Sell. Smoothness of spectral subbundles and reducibility of quasiperiodic linear differential systems. J. Differential Equations, 41(2):262-288, 1981.

[31] À. Jorba, R. de la Llave, and M. Zou. Lindstedt series for lower-dimensional tori. In Hamiltonian systems with three or more degrees of freedom (S'Agaró, 1995), volume 533 of NATO Adv. Sci. Inst. Ser. C Math. Phys. Sci., pages 151-167. 1999.

[32] À. Jorba and E. Olmedo. On the Computation of Reducible Invariant Tori on a Parallel Computer. SIAM J. Appl. Dyn. Syst., 8(4):1382-1404, 2009.

[33] À. Jorba and C. Simó. On the reducibility of linear differential equations with quasiperiodic coefficients. J. Differential Equations, 98(1):111-124, 1992. 
[34] À. Jorba and C. Simó. On quasi-periodic perturbations of elliptic equilibrium points. SIAM J. Math. Anal., 27(6):1704-1737, 1996.

[35] À. Jorba and J. Villanueva. On the normal behaviour of partially elliptic lower-dimensional tori of Hamiltonian systems. Nonlinearity, 10(4):783-822, 1997.

[36] À. Jorba and J. Villanueva. On the persistence of lower-dimensional invariant tori under quasi-periodic perturbations. J. Nonlinear Sci., 7(5):427-473, 1997.

[37] À. Jorba and J. Villanueva. Numerical computation of normal forms around some periodic orbits of the restricted three-body problem. Phys. D, 114(3-4):197-229, 1998.

[38] A.N. Kolmogorov. On conservation of conditionally periodic motions for a small change in Hamilton's function. Dokl. Akad. Nauk SSSR (N.S.), 98:527-530, 1954.

[39] R. Krikorian. Réductibilité presque partout des flots fibrés quasi-périodiques à valeurs dans des groupes compacts. Ann. Sci. École Norm. Sup. (4), 32(2):187-240, 1999.

[40] S.B. Kuksin. Perturbation of conditionally periodic solutions of infinite-dimensional Hamiltonian systems. Izv. Akad. Nauk SSSR Ser. Mat., 52(1):41-63, 240, 1988.

[41] Y. Li and Y. Yi. Persistence of hyperbolic tori in Hamiltonian systems. J. Differential Equations, 208(2):344-387, 2005.

[42] P. Lochak and C. Meunier. Multiphase Averaging for Classical Systems, volume 72 of Appl. Math. Sci. Springer, New York, 1988.

[43] V.K. Melnikov. On certain cases of conservation of almost periodic motions with a small change of the Hamiltonian function. Dokl. Akad. Nauk SSSR, 165:1245-1248, 1965.

[44] V.K. Melnikov. A certain family of conditionally periodic solutions of a Hamiltonian system. Dokl. Akad. Nauk SSSR, 181:546-549, 1968.

[45] L. Michelotti. Intermediate classical dynamics with applications to beam physics. Wiley Series in Beam Physics and Accelerator Technology. John Wiley \& Sons Inc., New York, 1995. A Wiley-Interscience Publication.

[46] J. Moser. On invariant curves of area-preserving mappings of an annulus. Nachr. Akad. Wiss. Göttingen Math.-Phys. Kl. II, 1962:1-20, 1962.

[47] J. Moser. A rapidly convergent iteration method and non-linear differential equations. II. Ann. Scuola Norm. Sup. Pisa (3), 20:499-535, 1966.

[48] J. Moser. A rapidly convergent iteration method and non-linear partial differential equations. I. Ann. Scuola Norm. Sup. Pisa (3), 20:265-315, 1966. 
[49] J. Moser. Convergent series expansions for quasi-periodic motions. Math. Ann., 169:136176, 1967.

[50] J. Moser and J. Pöschel. An extension of a result by Dinaburg and Sinaĭ on quasiperiodic potentials. Comment. Math. Helv., 59(1):39-85, 1984.

[51] A.I. Neĭshtadt. The separation of motions in systems with rapidly rotating phase. Prikl. Mat. Mekh., 48(2):197-204, 1984.

[52] M. Ollé, J.R. Pacha, and J. Villanueva. Kolmogorov-Arnold-Moser aspects of the periodic Hamiltonian Hopf bifurcation. Nonlinearity, 21(8):1759-1811, 2008.

[53] R. Paskauskas, C. Chandre, and T. Uzer. Dynamical bottlenecks to intramolecular energy flow. Phys. Rev. Let., 100(8):083001, 2008.

[54] J. Pöschel. On elliptic lower-dimensional tori in Hamiltonian systems. Math. Z., 202(4):559-608, 1989.

[55] J. Pöschel. A lecture on the classical KAM theorem. In Smooth ergodic theory and its applications (Seattle, WA, 1999), volume 69 of Proc. Sympos. Pure Math., pages 707-732. Amer. Math. Soc., 2001.

[56] J. Puig. Reducibility of linear differential equations with quasi-periodic coefficients: a survey. Preprint available electronically at http://www.mal.upc.edu/ jpuig/ preprints/qpred.ps.

[57] H. Rüssmann. On optimal estimates for the solutions of linear partial differential equations of first order with constant coefficients on the torus. In Dynamical systems, theory and applications (Rencontres, Battelle Res. Inst., Seattle, Wash., 1974), pages 598-624.Lecture Notes in Phys., Vol. 38. Springer, Berlin, 1975.

[58] H. Rüssmann. On a new proof of Moser's twist mapping theorem. In Proceedings of the Fifth Conference on Mathematical Methods in Celestial Mechanics (Oberwolfach, 1975), Part I, number 1, pages 19-31, 1976.

[59] H. Rüssmann. Invariant tori in non-degenerate nearly integrable Hamiltonian systems. Regul. Chaotic Dyn., 6(2):119-204, 2001.

[60] D. Salamon and E. Zehnder. KAM theory in configuration space. Comment. Math. Helv., 64(1):84-132, 1989.

[61] M.B. Sevryuk. KAM-stable Hamiltonians. J. Dynam. Control Systems, 1(3):351-366, 1995. 
[62] M.B. Sevryuk. Invariant tori of Hamiltonian systems that are nondegenerate in the sense of Rüssmann. Dokl. Akad. Nauk, 346(5):590-593, 1996.

[63] M.B. Sevryuk. The lack-of-parameters problem in the KAM theory revisited. In Hamiltonian systems with three or more degrees of freedom (S'Agaró, 1995), volume 533 of NATO Adv. Sci. Inst. Ser. C Math. Phys. Sci., pages 568-572. Kluwer Acad. Publ., 1999.

[64] M.B. Sevryuk. Invariant tori in quasi-periodic non-autonomous dynamical systems via Herman's method. Discrete Contin. Dyn. Syst., 18(2-3):569-595, 2007.

[65] A.M. Tarquis, J.C. Losada, R.M. Benito, and F. Borondo. Multifractal analysis of tori destruction in a molecular Hamiltonian system. Phys. Rev. E, 65(1):016213, 2001.

[66] J. Xu and J. You. Persistence of lower-dimensional tori under the first Melnikov's nonresonance condition. J. Math. Pures Appl. (9), 80(10):1045-1067, 2001.

[67] E. Zehnder. Generalized implicit function theorems with applications to some small divisor problems. II. Comm. Pure Appl. Math., 29(1):49-111, 1976. 